\title{
Electronic Properties of Various Two-Dimensional Materials
}

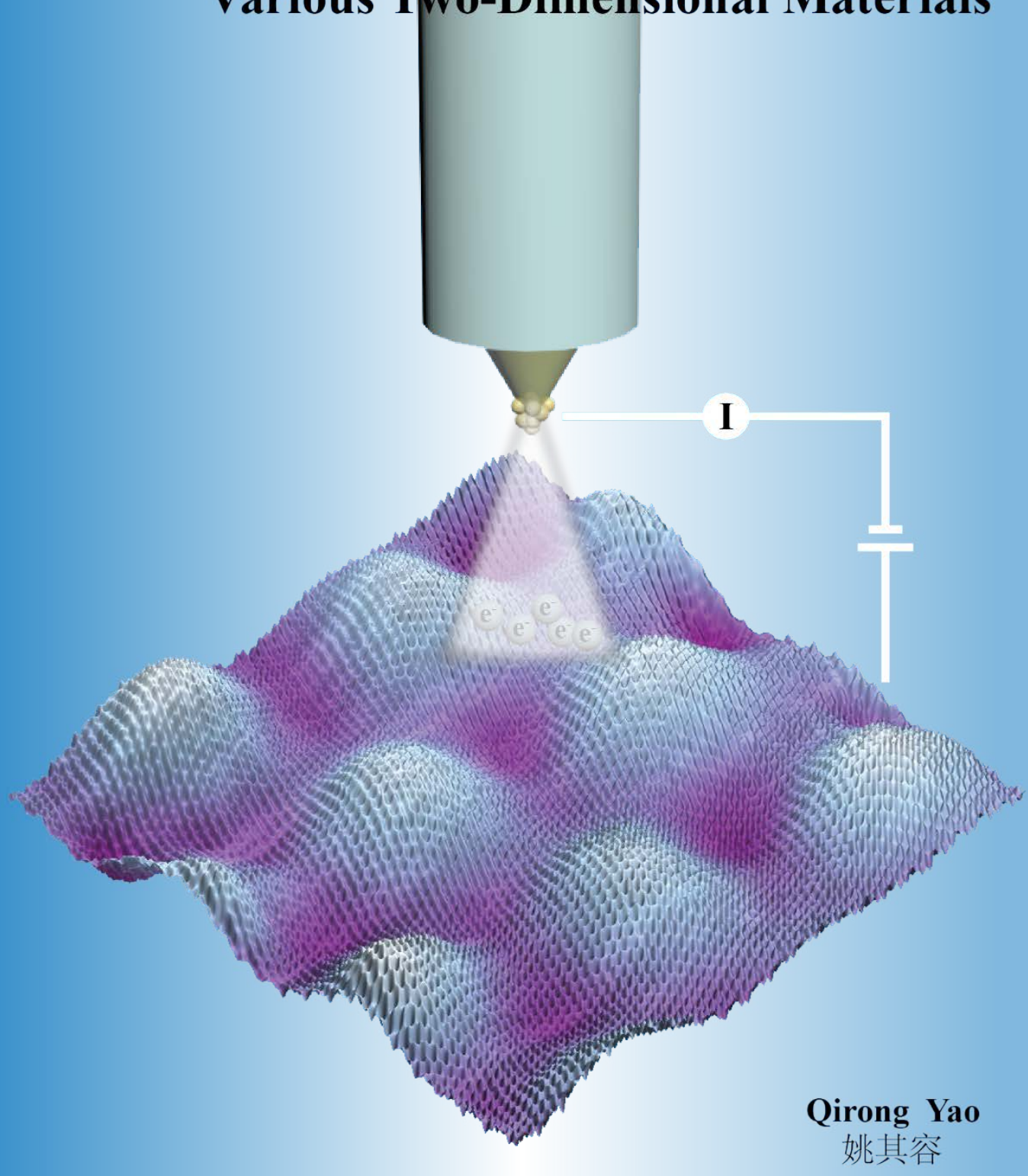


ELECTRONIC PROPERTIES OF VARIOUS TWODIMENSIONAL MATERIALS

Qirong Yao 


\section{Graduation Committee:}

Chairman / secretary:

Supervisor:

Committee Members:
Prof. dr. J.L. Herek

Prof. dr. ir. H.J.W. Zandvliet

Prof. dr. M.A. Stöhr

Prof. dr. O. Gurlu

Prof. dr. ir. J.W.M. Hilgenkamp

Prof. dr. ir. J.E. ten Elshof

Dr. A. van Houselt 


\title{
ELECTRONIC PROPERTIES OF VARIOUS TWO- DIMENSIONAL MATERIALS
}

\author{
DISSERTATION \\ to obtain \\ the degree of doctor at the Universiteit Twente, \\ on the authority of the rector magnificus, \\ Prof.dr. T.T.M. Palstra,
}

on account of the decision of the graduation committee

to be publicly defended

on Wednesday 11 September 2019 at 12.45

by

Qirong Yao

born on 16 October 1989

in Hubei, China 
This dissertation has been approved by the supervisor:

Prof. dr. ir. H.J.W. Zandvliet

Cover design: Qirong Yao

Printed by: Gildeprint drukkerijen, Enschede, The Netherlands.

ISBN: 978-90-365-4846-5

DOI: $10.3990 / 1.9789036548465$

(C) 2019 Qirong Yao, The Netherlands. All rights reserved. No parts of this thesis may be reproduced, stored in a retrieval system or transmitted in any form or by any means without permission of the author. Alle rechten voorbehouden. Niets uit deze uitgave mag worden vermenigvuldigd, in enige vorm of op enige wijze, zonder voorafgaande schriftelijke toestemming van de auteur. 


\section{Contents}

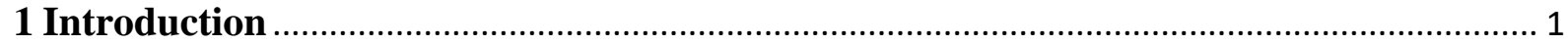

1.1 Preamble

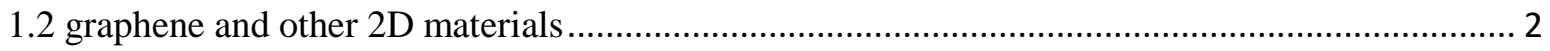

1.3 Modification of electronic structure in 2D materials.................................................................. 3

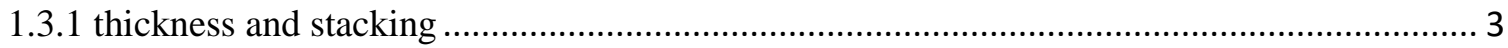

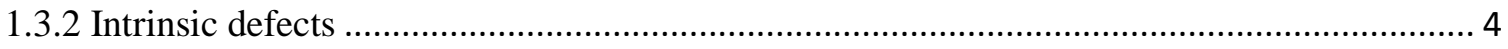

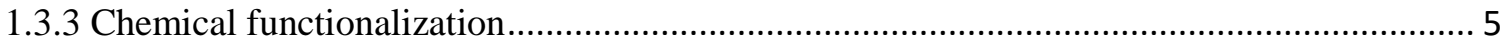

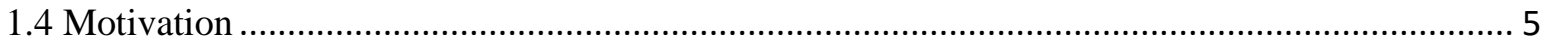

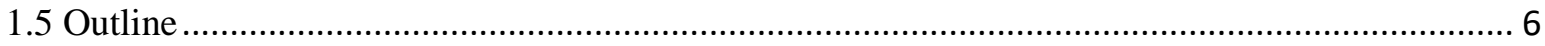

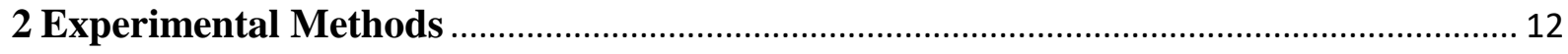

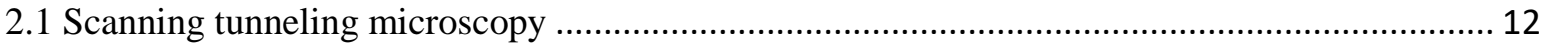

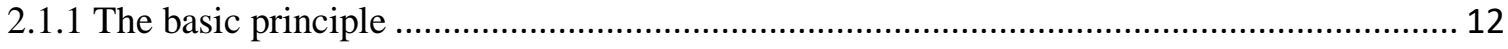

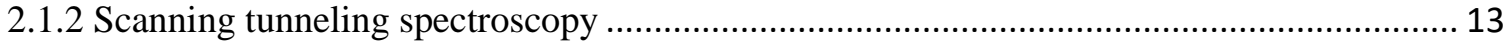

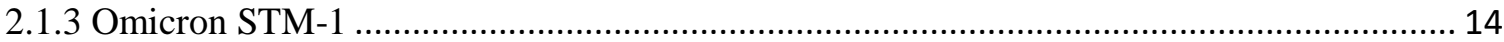

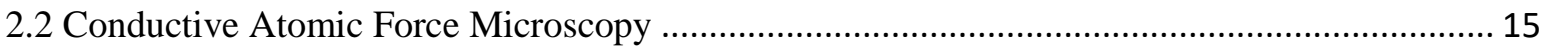

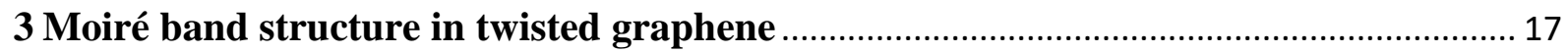

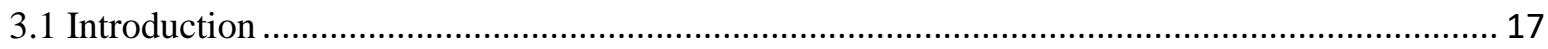

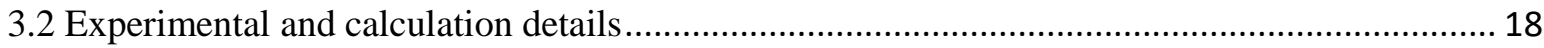

3.3 Spatial resolved electronic structure of twisted graphene ..................................................... 19

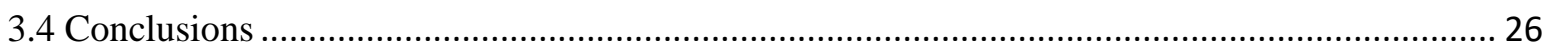

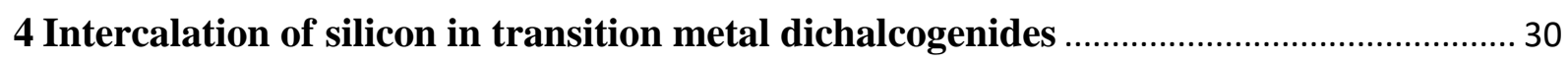

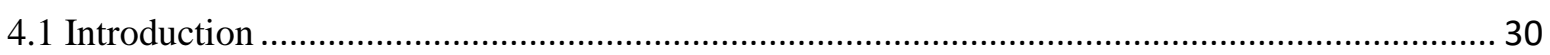

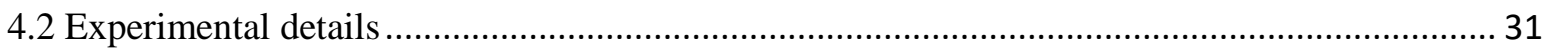

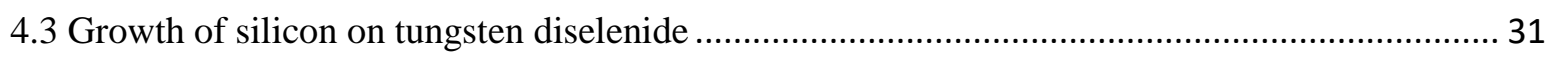

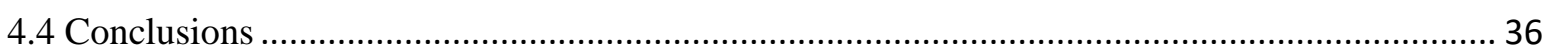

5 Effect of substrate and hydrogenation on germanene ............................................... 42

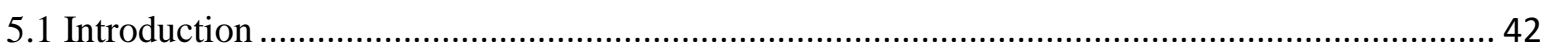

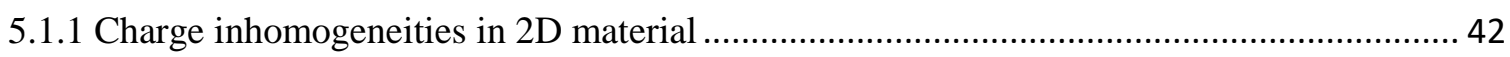

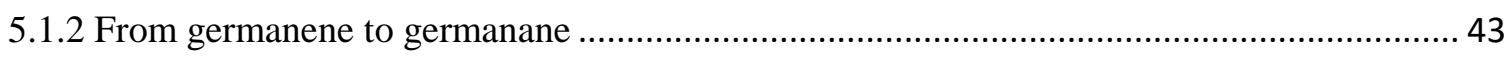

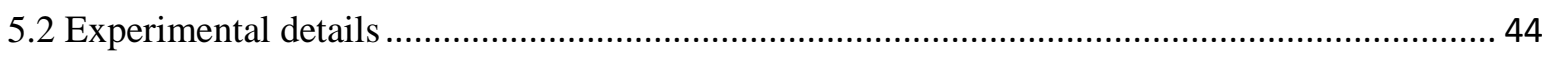

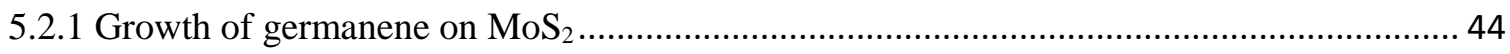

5.2.2 Hydrogenation of germanene terminated on $\mathrm{Ge}_{2} \mathrm{Pt}$ nanocrystal.......................................... 45

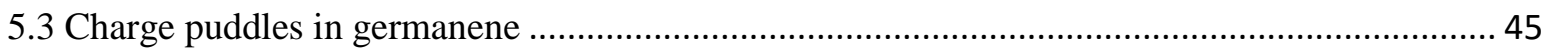

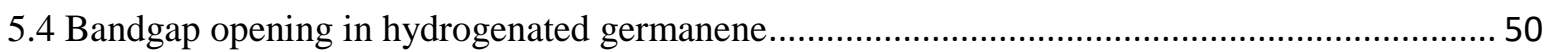




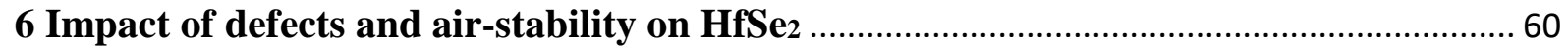

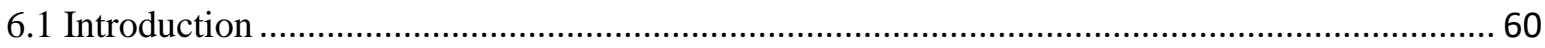

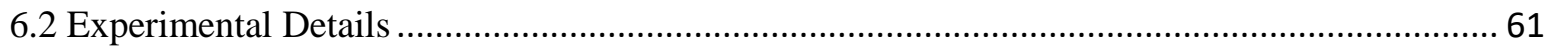

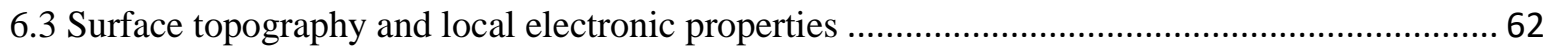

6.4 Schottky barrier height and Fermi level pinning of the HfSe $e_{2}$ crystal....................................... 68

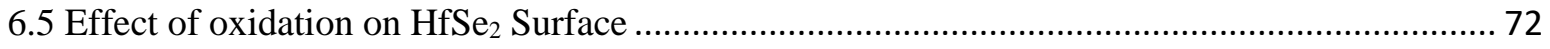

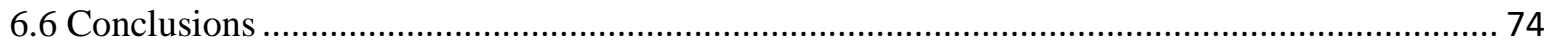

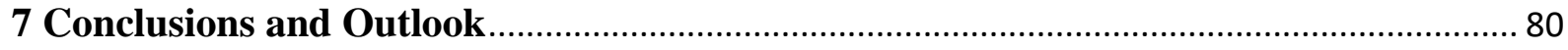

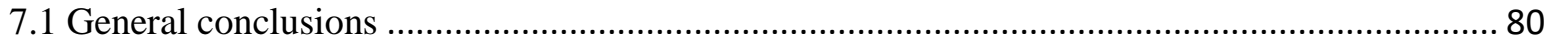

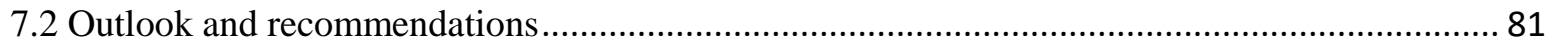

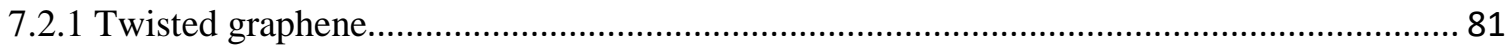

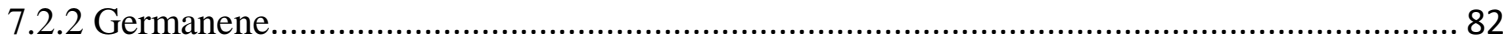

Summary

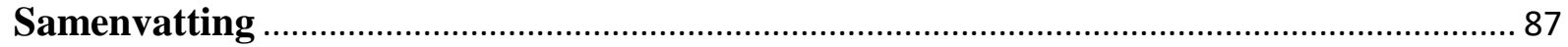

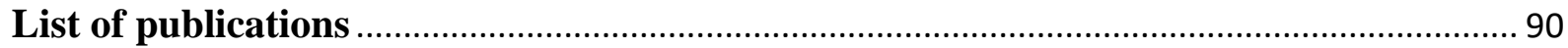

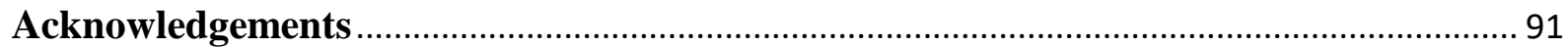





\section{Introduction}

\subsection{Preamble}

Graphene, a single layer of carbon atoms packed in a honeycomb structure, was isolated from graphite for the first time by Andre Geim and Konstantin Novoselov in 2004. ${ }^{[1]}$ Due to the excellent electrical, thermal, and mechanical properties, graphene has been widely explored in various areas of physical and chemical applications, including electronic devices, chemical sensors, catalysis, batteries and other energy conversion systems. ${ }^{[2-5]}$ The discovery of graphene opens the door to a total new research field and sets off the continuous waves on the vigorous scientific research of two-dimensional (2D) materials. ${ }^{[6-9]}$ The family of 2D materials involves, metals, semimetals, semiconductors, insulators and even superconductors as shown in Figure 1.1. ${ }^{[10]}$ Among them, most conventional 2D materials have been derived from a limited library of bulk solids consisting of stacked, weakly bound sheets (for example, graphite, $\mathrm{MoS}_{2}$, hexagonal boron nitride and black phosphorus). In contrast, the growth of an entirely new class of synthetic 2D materials, such as silicene, germanene and stanene, expands the variety of materials with new, tailorable properties based on their composition and substrate. ${ }^{[11-13]}$

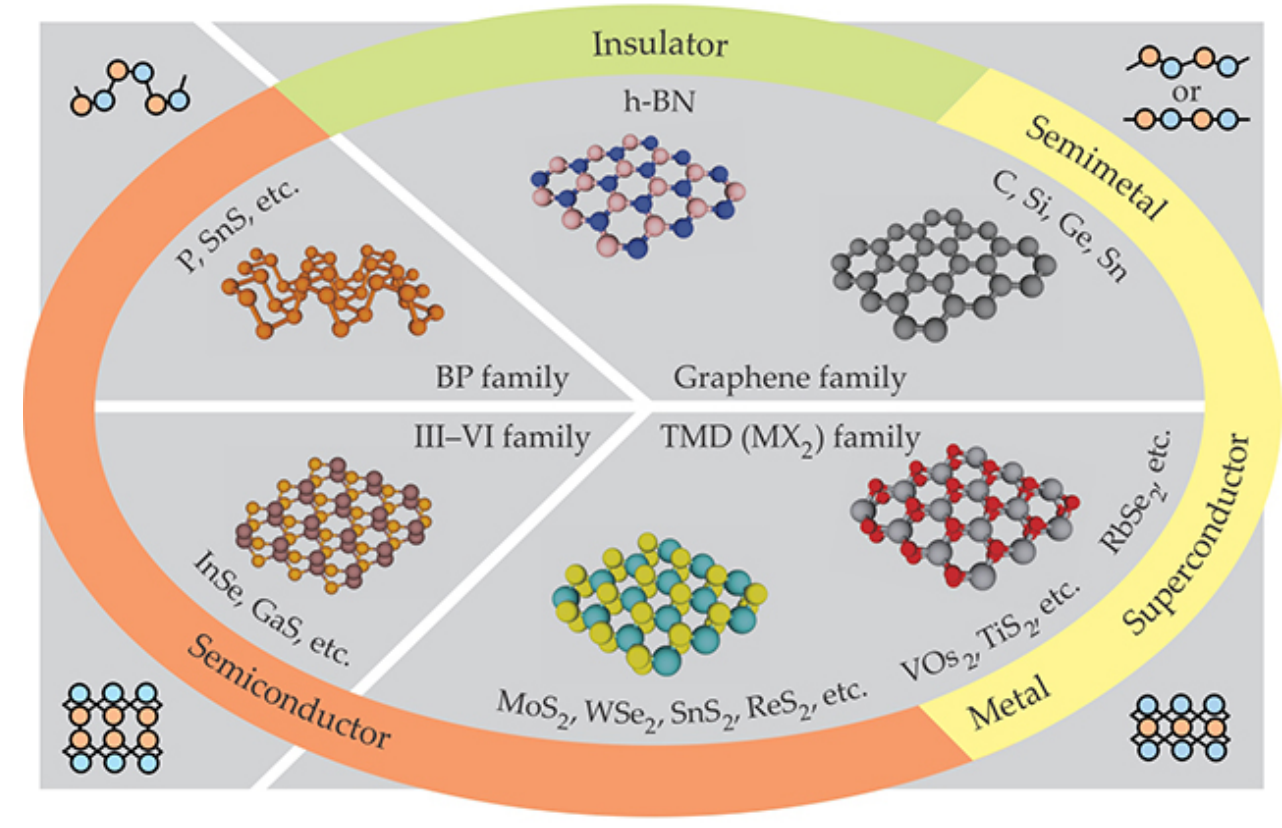

Figure 1.1: The family of two-dimensional materials ${ }^{[10]}$ 


\section{2 graphene and other $2 \mathrm{D}$ materials}

Graphene, a monolayer of $\mathrm{sp}^{2}$ hybridized carbon atoms arranged in honeycomb structure, is shown in Figure 1.2a. The honeycomb lattice of graphene consists of two sub-lattices with a lattice constant of $2.46 \AA$, while the C-C distance is $a=1.42 \AA$. Graphene is a two-dimensional Dirac material. The electrons in graphene behave as massless relativistic particles obeying the Dirac equation, i.e. the relativistic variant of the Schrödinger equation. ${ }^{[2,14]}$ In the vicinity of the Fermi energy level, the dispersion relation of the electrons is linear resulting in a cone-like band structure referred to as a Dirac cone.

Silicene and germanene, i.e. the silicon and germanium analogues of graphene, are 2D buckled Dirac materials. ${ }^{[12,15-17]}$ Similar to graphene, the electrons in silicene and germanene near the Fermi energy level also behave as the massless relativistic Dirac fermions. The $\pi$ and $\pi *$ bands are linear and cross at the Fermi level and therefore silicene and germanene are both semimetals. Silicene and germanene exhibit a combined $\mathrm{sp}^{2}-\mathrm{sp}^{3}$ hybridization, rather than a perfect $\mathrm{sp}^{2}$ bonding as in graphene. Figure 1.2b shows this honeycomb structure and the buckled nature, where one half of the atoms of the unit cell is located in a lower plane and the other half is located in a higher plane. Each $\mathrm{Si}(\mathrm{Ge})$ atom is covalently bonded with three other $\mathrm{Si}(\mathrm{Ge})$ atoms, resulting in a simple hexagonal unit cell. The predicted buckling of silicene and germanene is $0.44 \AA$ and $0.64 \AA$, respectively ${ }^{[15]}$ Buckled silicene and germanene are stable, whereas perfectly planar silicene and germanene sheets have imaginary phonon modes in a large portion of the Brillouin zone and are therefore not stable. Another difference with graphene is that silicene and germanene have a much larger spin-orbit coupling owing to their larger atomic number.

The spin-orbit gap of silicene and germanene has been calculated to be $\sim 1.5 \mathrm{meV}$ and $24 \mathrm{meV}$ respectively, which is much larger than that of graphene (only a few $\mu \mathrm{eV}$ ). ${ }^{[18,19]}$

Since the absence of a bandgap in graphene limits its application in the semiconductor industry, the layered transition metal dichalcogenides (TMDs) have received quite some attention during the last decade. ${ }^{[20-22]}$ The formula for transition metal dichalcogenides is $\mathrm{MX}_{2}$, where $\mathrm{M}$ refers to a transition metal atom and $\mathrm{X}$ to a chalcogen atom. TMDs consist of a hexagonal packed layer of $\mathrm{M}$ atoms sandwiched by two layers of $\mathrm{X}$ atoms. The intralayer $\mathrm{M}-\mathrm{X}$ bonds are predominantly covalent in nature, whereas the sandwiched layers are coupled by weak van der Waals forces. The structure model is shown in Figure 1.2c. The semiconducting 2D TMDs, 
including $\mathrm{MoS}_{2}, \mathrm{MoSe}_{2}, \mathrm{MoTe}_{2}, \mathrm{WS}_{2}$, WSe 2 and $\mathrm{HfSe}_{2}$, have emerged as promising materials for a wide range of applications. ${ }^{\text {[22, 23] }}$

(a) Graphene

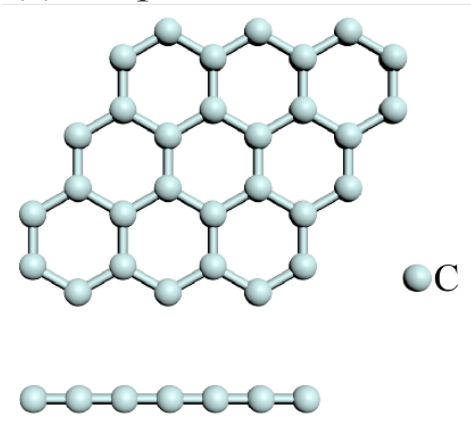

(b)Silicene/Germanene

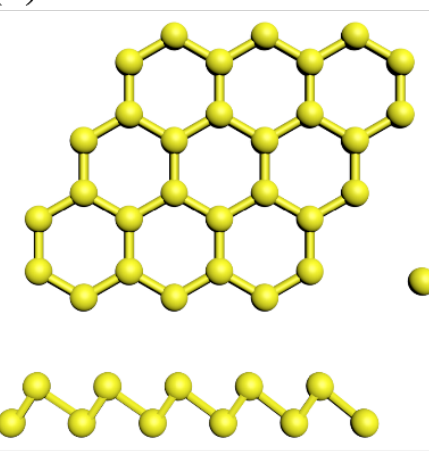

(c) $\mathrm{MX}_{2}$
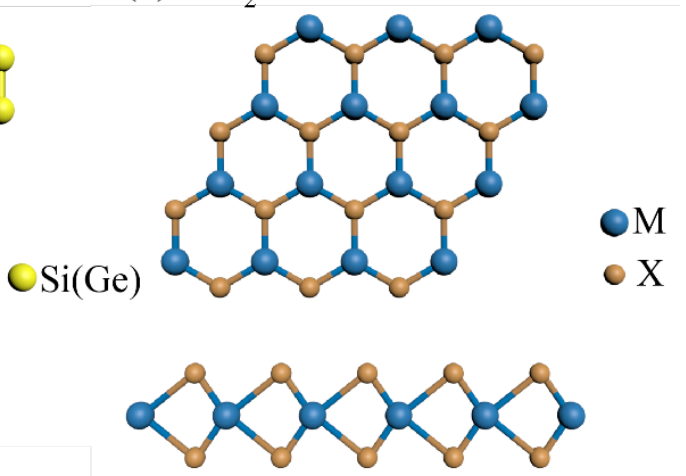

Figure 1.2: Structural models of graphene in (a), silicene/germanene in (b), and $\mathrm{MX}_{2}$ in (c).

\subsection{Modification of electronic structure in 2D materials}

\subsection{1 thickness and stacking}

In $2 \mathrm{D}$ materials, both the thickness as well as the stacking order of the $2 \mathrm{D}$ flake affects the electronic band structure. The properties of 2D materials are a function of the number of layers. For example, a single-layer graphene has linearly dispersing energy bands resulting in a perfect Dirac cone, but for a Bernal stacked bilayer of graphene the energy bands are parabolic in the direct vicinity of the Dirac point ${ }^{[2,24]}$. The Bernal stacked bilayer corresponds to a twist angle of 0 degrees. For small twist angles the two Dirac cones of both graphene layers move towards each other in reciprocal space resulting in hybridization and the formation of Van Hove singularities.

The transition metal dichalcogenides behave differently. For instance, bulk $\mathrm{MoS}_{2}$ has an indirect band gap of $1.29 \mathrm{eV}$. However, when it is thinned to a monolayer, the crystal transfers into a direct bandgap semiconductor. ${ }^{[25]}$ A strong photoluminescence emerges in the monolayer $\mathrm{MoS}_{2 .}{ }^{[26]}$ Black phosphorus has an intrinsic thickness-dependent direct band gap. The band gap varies from $0.3 \mathrm{eV}$ for bulk to $2.0 \mathrm{eV}$ for a monolayer, which covers a broad range of the solar spectrum and consequently makes this material suitable for numerous optoelectronic applications. ${ }^{[27,28]}$

Stacking is also a powerful approach to tailor the electronic properties of 2D materials. When two monolayers of 2D materials are stacked on top of each other to create a bilayer structure, 
their lattices do not necessarily align with each other. For example, in the most energetically favorable form of bilayer graphene, the layers are shifted over the length of one carbon-carbon bond with respect to each other. This combination is known as AB or Bernal stacked form. On the other hand, AA stacking where two layers are exactly aligned or a twisted structure where one layer is rotated relative to the other is also possible. When a third layer is added to the top of $\mathrm{AB}$ stacked bilayer graphene it can align with the bottom layer to create an ABA stacked trilayer graphene or it can be shifted one more carbon-carbon bond length and create an $A B C$ stacked trilayer graphene. ABA and ABC stacked forms of trilayer graphene display qualitatively different electronic properties. ${ }^{[29-31]}$ The dispersion of ABA trilayer is a combination of the linear dispersion of a single layer graphene and the quadratic relation of bilayer graphene; whereas the dispersion of $\mathrm{ABC}$ trilayer is approximately cubic, with its conductance and valence bands touching at a point close to the highly symmetric K- and $\mathrm{K}^{\prime}$ points. By applying an external electric field, a large band gap can be formed in the trilayer graphene with the ABC stacking order rather than the ABA stacking order. Especially, twisted bilayer graphene can result in the large moiré superlattices accompanied by new phenomena, such as stacking-dependent Van Hove singularities near the Fermi energy, unconventional superconductivity and insulating behavior. ${ }^{[32-34]}$ In bilayer $\mathrm{MoS}_{2}$, proper manipulation of stacking orders can break the inversion symmetry and suppress interlayer hopping, introducing strong valley and spin polarizations that cannot be achieved in natural $\mathrm{MoS}_{2}$ bilayers with Bernal stacking. ${ }^{[35,36]}$ Furthermore, flat bands at the valence band edge of twisted bilayer TMDs $\left(\mathrm{MoS}_{2}, \mathrm{WS}_{2}, \mathrm{MoSe}_{2}, \mathrm{WS} \mathrm{W}_{2}\right)$ are predicted to occur. ${ }^{[37]}$

\subsubsection{Intrinsic defects}

Atomic defects, such as edges, vacancies, and lattice disorder are localized heterogeneities that can be formed without perturbing the native lattice structure of $2 \mathrm{D}$ materials. Defects and vacancies are ubiquitous in 2D materials and can have a significant impact on the electrical properties of these materials. Take graphene as an example, when scrutinized over large-scales it contains point defects, edges, and grain boundaries. The point defects give rise to localized states near the Fermi level, leading to protrusions in STM images. ${ }^{[38]}$ They also act as scattering centers for electron waves. Thus, one can anticipate that these defects will result in the reduction of electronic mobility of graphene. ${ }^{[39]}$ Some vacancy-type and Stone-Wales defects can open a local bandgap (up to $0.3 \mathrm{eV}$ ) in graphene. ${ }^{[40]}$ For the grain boundaries, they can suppress the conductivity of both the electron and hole-type charge carriers and trigger a local-doping effect 
in graphene. In monolayer $\mathrm{MoS}_{2}$, sulfur vacancies are the most common defects during exfoliation or chemical vapor deposition growth. These sulfur vacancies can introduce unpaired electrons in the lattice and consequently make the material be n-doped. If the material is sulfur atom rich or the molybdenum atom poor, the material exhibits p-type behavior. The grain boundaries in $\mathrm{MoS}_{2}$ can be either sulfur-deficient or molybdenum-deficient, so it means the inplane electrical conductivity can be modulated by such kind of defects. ${ }^{[41]}$

\subsubsection{Chemical functionalization}

Chemical functionalization is in general an efficient way to tune the electronic properties of 2D materials. Particularly, hydrogenation of 2D materials has shown to be a promising method. ${ }^{[42-}$

${ }^{45]}$ In principle, when the graphene surface in hydrogenated, it will change the hybridization of carbon atoms from $\mathrm{sp}^{2}$ to $\mathrm{sp}^{3}$, thus removing the conducting $\pi$-bands and opening up an energy gap. It has also been show that a superlattice structure of graphene-like islands by patterned adsorption of atomic hydrogen onto a moiré superlattice of graphene grown on an $\operatorname{Ir}(111)$ substrate can induce a bandgap in the electronic band structure due to confinement. ${ }^{[43]}$ As mentioned before, the sulfur vacancies in $\mathrm{MoS}_{2}$ flakes can cause the presence of unsaturated electrons in the surrounding molybdenum atoms and act as electrons donors, which is responsible for the n-type doping of $\mathrm{MoS}_{2}$. By exposing a single layer $\mathrm{MoS}_{2}$ to atomic hydrogen at room temperature, hydrogen atoms will passivate sulfur vacancies and consequently the electronic properties of single layer $\mathrm{MoS}_{2}$ can be tuned from intrinsic electron (n) to hole (p) doping without degrading the quality of $\mathrm{MoS}_{2}$ flakes. ${ }^{[44]}$ Furthermore, by employing firstprinciples calculations, it has been found that the intrinsic bilayer silicene can be transferred from a semiconductor with an indirect band gap to a direct-gap semiconductor with a widely tunable band gap (from 1 to $1.5 \mathrm{eV}$ ) by hydrogenation, which is suitable for solar applications. ${ }^{[45]}$

\subsection{Motivation}

Measuring the physical properties of 2D materials and correlating the spatial variation of these physical properties to the structure provides a route to better understand these materials. A very powerful tool for probing the surface structure and local electronic properties is scanning tunneling microscopy. Scanning tunneling spectroscopy allows the observation of electronic spectral properties with a resolution down to the atomic scale. In this thesis, we mainly employed scanning tunneling microscopy and scanning tunneling spectroscopy to study the structure and electronic properties of different types of 2D materials. Such fundamental 
research can help us to understand the 2D materials at the nano-scale, and pave the way towards the application of these materials in future electronic devices.

\subsection{Outline}

The following chapter describes the experimental techniques that have been used in this thesis. Chapter 3 deals with the structural and electronic properties of twisted graphene. We show that for small twist angles the electronic structure in the vicinity of the Dirac point alters significantly. Two Van Hove singularities form, one located just below the Dirac point and one located just above the Dirac point. Spatial maps of the local density of states reveals a honeycomb structure, which is composed of two sub-lattices.

In Chapter 4 the growth of silicon on TMDs surfaces is studied. Unfortunately, we found that it is not possible to grow silicene on TMD substrates. The deposited silicon atoms do not reside on the TMD surface, but rather intercalate between the TMD sheets.

Chapter 5 deals with the growth and characterization of germanene on different substrates. We show that electron-hole puddles are present in the germanene on $\mathrm{MoS}_{2}$ system. Furthermore, we also show that the band structure of germanene can be modified by chemical functionalization. The adsorption of hydrogen on germanene results in the formation of a sizeable bandgap of $\sim 0.5 \mathrm{eV}$. In Chapter 6 we show and explain why the charge mobilities in $\mathrm{HfSe}_{2}$ are so low. In addition, we also elaborate stability of $\mathrm{HfSe}_{2}$ at ambient conditions.

We end this thesis with conclusions and an outlook. 


\section{Bibliography}

[1] Novoselov, K. S.; Geim, A. K.; Morozov, S. V.; Giacometti, V.; Jiang, D.; Zhang, Y.; Dubonos, S. V.; Grigorieva, I. V.; Firsov. A. A. Electric Field Effect in Atomically Thin Carbon Films. Science 2004, 306, 666-669.

[2] Castro Neto, A. H.; Guinea, F.; Peres, N. M. R.; Novoselov, K. S.; Geim, A. K. The Electronic Properties of Graphene. Rev. Mod. Phys. 2009, 81, 109-162.

[3] Shao, Y.; Wang, J.; Wu, H.; Liu, J.; Aksay, I. A.; Lin, Y. Graphene Based Electrochemical Sensors and Biosensors. Electroanalysis 2010, 22, 1027-1036.

[4] Bonaccorso, F.; Colombo, L.; Yu, G.; Stoller, M.; Tozzini, V.; Ferrari, A. C.; Ruoff, R. S.; Pellegrini, V. Graphene, Related Two-Dimensional Crystals, and Hybrid Systems for Energy Conversion and Storage. Science 2015, 347, 1246501.

[5] Huang, X.; Yin, Z.; Wu, S.; Qi, X.; He, Q.; Zhang, Q.; Yan, Q.; Boey, F.; Zhang, H. Graphen-Based Materials: Synthesis, Characterization, Properties, and Applications. Small 2011, 7, 1876-1902.

[6] Xu, M.; Liang, T.; Shi, M.; Chen, H. Graphene-Like Two-Dimensional Materials. Chem. Rev. 2013, 113, 3766-3798.

[7] Butler, S. Z.; Hollen, S. M.; Cao, L.; Cui, Y.; Gupta, J. A.; Gutierrez, H. R.; Heinz, T. F.; Hong, S. S.; Huang, J.; Ismach, A. F., et al. Progress, Challenges, and Opportunities in TwoDimensional Materials Beyond Graphene. ACS Nano 2013, 7, 2898-2926.

[8] Lv, R.; Robinson, J. A.; Schaak, R. E.; Sun, D.; Sun, Y.; Mallouk, T. E.; Terrones, M. Transition Metal Dichalcogenides and Beyond: Synthesis, Properties, and Applications of Single- and Few-Layer Nanosheets. Acc. Chem. Res. 2015, 48, 56-64.

[9] Liu, H.; Du, Y.; Deng, Y.; Ye, P. D. Semiconducting Black Phosphorus: Synthesis, Transport Properties and Electronic Applications. Chem. Soc. Rev., 2015, 44, 2732-2743. 
[10] Ajayan, P.; Kim, P.; Banerjee, K. Two-Dimensional Van der Waals Materials. Phys. Today 2016, 69, 38.

[11] Kara, A.; Enriquez, H.; Seitsonen, A. P.; Lew Yan Voon L. C.; Vizzini, S.; Aufray, B.; Oughaddou, H. A Review on Silicene - New Candidate for Electronics. Surf. Sci. Rep. 2012, 67, 1-18.

[12] Acun, A.; Zhang, L.; Bampoulis, P.; Farmanbar, M.; Houselt, A. V.; Rudenko, A. N.; Lingenfelder, M.; Brocks, G.; Poelsema, B.; Katsnelson, M. I.; Zandvliet, H. J. W. Germanene: the Germanium Analogue of Graphene. J. Phys.: Condens. Matter 2015, 27, 443002.

[13] Zhu, F.; Chen, W.; Xu, Y.; Gao, C.; Guan, D.; Liu, C.; Qian, D.; Zhang, S.; Jia, J. Epitaxial Growth of Two-Dimensional Stanene. Nat. Mater. 2015, 14, 1020-1025.

[14] Geim, A. K. Graphene: Status and Prospects. Science 2009, 324, 1530-1534.

[15] Cahangirov, S.; Topsakal, M.; Aktürk, E.; Șahin, H.; Ciraci, S. Two- and OneDimensional Honeycomb Structures of Silicon and Germanium. Phys. Rev. Lett. 2009, 102, 236804.

[16] Molle, A.; Goldberger, J.; Houssa, M.; Xu, Y.; Zhang, S.; Akinwande, D. Buckled TwoDimensional Xene Sheets. Nat. Mater. 2017, 16, 163-169.

[17] Fleurence, A.; Friedlein, R.; Ozaki, T.; Kawai, H.; Wang, Y.; Yamada-Takamura, Y. Experimental Evidence for Epitaxial Silicene on Diboride Thin Films. Phys. Rev. Lett. 2012, 108, 245501.

[18] Liu, C.; Feng, W.; Yao, Y. Quantum Spin Hall Effect in Silicene and Two-Dimensional Germanium. Phys. Rev. Lett. 2011, 107, 076802.

[19] Sichau, J.; Prada, M.; Anlauf, T.; Lyon, T. J.; Bosnjak, B.; Tiemann, L.; Blick, R.H. Resonance Microwave Measurements of an Intrinsic Spin-Orbit Coupling Gap in Graphene: A Possible Indication of a Topological State. Phys. Rev. Lett. 2019, 122, 046403. 
[20] Chhowalla, M.; Shin, H. S.; Eda, G.; Li, L.; Loh, K. P.; Zhang, H. The Chemistry of Two-Dimensional Layered Transition Metal Dichalcogenide Nanosheets. Nat. Chem. 2013, 5, 263-275.

[21] Johari, P.; Shenoy, V. B. Tuning the Electronic Properties of Semiconducting Transition Metal Dichalcogenides by Applying Mechanical Strains. ACS Nano 2012, 6, 5449-5456.

[22] Duan, X.; Wang, C.; Pan, A.; Yu, R.; Duan, X. Two-Dimensional Transition Metal Dichalcogenides as Atomically Thin Semiconductors: Opportunities and Challenges. Chem. Soc. Rev., 2015, 44, 8859-8876.

[23] Mleczko, M. J.; Zhang, C.; Lee, H. R.; Kuo, H.; Magyari-Köpe, B.; Moore, R. G.; Shen, Z.; Fisher, I. R.; Nishi, Y.; Pop, E. HfSe 2 and ZrSe2: Two-dimensional Semiconductors with Native High- $\kappa$ Oxides. Sci. Adv. 2017, 3, e1700481.

[24] Ohta, T.; Bostwick, A.; McChesney, J. L.; Seyller, T.; Horn, K.; Rotenberg, E. Interlayer Interaction and Electronic Screening in Multilayer Graphene Investigated with AngleResolved Photoemission Spectroscopy. Phys. Rev. Lett. 2007, 98, 206802.

[25] Mak, K. F.; Lee, C.; Hone, J.; Shan, J.; Heinz, T. F. Atomically Thin $\mathrm{MoS}_{2}$ : A New Direct-Gap Semiconductor. Phys. Rev. Lett. 2010, 105, 136805.

[26] Splendiani, A.; Sun, L.; Zhang, Y.; Li, T.; Kim, J.; Chim, C.; Galli, G.; Wang, F. Emerging Photoluminescence in Monolayer MoS 2 . Nano Lett. 2010, 10, 1271-1275.

[27] Qiao, J.; Kong, X.; Hu, Z.; Yang, F.; Ji, W. High-Mobility Transport Anisotropy and Linear Dichroism in Few-Layer Black Phosphorus. Nat. Commun. 2014, 5, 4475.

[28] Li, L.; Yu, Y.; Ye, G. J.; Ge, Q.; Ou, X.; Wu, H.; Feng, D.; Chen, X. H.; Zhang, Y. Black Phosphorus Field-Effect Transistors. Nat. Nanotechnol. 2014, 9,372-377.

[29] Lui, C. H.; Li, Z.; Mak, K. F.; Cappelluti, E.; Heinz, T. F. Observation of An Electrically Tunable Band Gap in Trilayer Graphene. Nat. Phys. 2011, 7, 944-947. 
[30] Craciun, M. F.; Russo, S.; Yamamoto, M.; Oostinga, J. B.; Morpurgo, A. F.; and Tarucha, S. Trilayer Graphene is A Semimetal with a Gate-Tunable Band Gap. Nat. Nanotechnol. 2009, 4, 383-388.

[31] Bao, W.; Jing, L.; Jr, J. V.; Lee, Y.; Liu, G.; Tran, D. et al., Stacking-Dependent Band Gap and Quantum Transport in Trilayer Graphene. Nat. Phys. 2011, 7, 948-952.

[32] Li, G.; Luican, A.; Lopes dos Santos, J. M. B.; Castro Neto, A. H.; Reina, A.; Kong, J.; Andrei, E. Y. Observation of Van Hove Singularities in Twisted Graphene Layers. Nat. Phys. 2010, 6, 109-113.

[33] Cao, Y.; Fatemi, V.; Fang, S.; Watanabe, K.; Taniguchi, T.; Kaxiras, E.; Jarillo-Herrero, P. Unconventional Superconductivity in Magic-Angle Graphene Superlattices. Nature 2018, 556, 43-50.

[34] Cao, Y.; Fatemi, V.; Demir, A.; Fang, S. et. al., Correlated Insulator Behaviour at HalfFilling in Magic-Angle Graphene Superlattices Nature 2018, 556, 80-84.

[35] Wu, S.; Ross, J. S.; Liu, G.; Aivazian, G.; Jones, A.; Fei, Z.; Zhu, W.; Xiao, D.; Yao, W.; Cobden, D.; Xu, X. Electrical Tuning of Valley Magnetic Moment Through Symmetry Control in Bilayer MoS 2 Nat. Phys. 2013, 9, 149-153.

[36] Liu, K.; Zhang, L.; Cao, T.; Jin, C.; Qiu, D.; Zhou, Q.; Zettl, A.; Yang, P.; Louie, S. G.; Wang, F. Evolution of Interlayer Coupling in Twisted Molybdenum Disulfide Bilayers. Nat. Commun. 2014, 5, 4966.

[37] Naik, M. H.; Jain, M. Ultraflatbands and Shear Solitons in Moiré Patterns of Twisted Bilayer Transition Metal Dichalcogenides. Phys. Rev. Lett. 2018, 121, 266401.

[38] Ugeda, M. M.; Ferna'ndez-Torre, D.; Brihuega, I.; Pou, P.; Martı'nez-Galera, A. J.; Pe'rez, R.; Go'mez-Rodrı' guez, J. M. Point Defects on Graphene on Metals. Phys. Rev. Lett. 2011, 107, 116803. 
[39] Chen, J.; Cullen, W. G.; Jang, C.; Fuhrer, M.S.; Williams, E. D. Defect Scattering in Graphene. Phys. Rev. Lett. 2009, 102, 236805.

[40] Banhart, F.; Kotakoski, J.; Krasheninnikov, A. V. Structural Defects in Graphene. ACS Nano, 2011, 5, 26-41.

[41] Lin, Z.; Carvalho, B. R.; Kahn, E.; Lv, R.; Rao, R.; Terrones. H.; Pimenta, M. A.; Terrones, M. Defect Engineering of Two-Dimensional Transition Metal Dichalcogenides. 2D Mater. 2016, 3, 022002.

[42] Elias, D. C.; Nair, R. R.; Mohiuddin, T. M. G.; Morozov, S. V.; Blake, P.; Halsall, M. P.; Ferrari, A. C.; Boukhvalov, D. W.; Katsnelson, M. I.; Geim, A. K.; Novoselov, K. S. Control of Graphene’s Properties by Reversible Hydrogenation: Evidence for Graphane. Science 2009, 323, 610-613.

[43] Balog, R.; Jørgensen, B.; Nilsson, L.; Andersen, M. et al., Bandgap Opening in Graphene Induced by Patterned Hydrogen Adsorption. Nat. Mater. 2010, 9, 315-319.

[44] Pierucci, D.; Henck, H.; Aziza, Z. B.; Naylor, C. H. et al., Tunable Doping in Hydrogenated Single Layered Molybdenum Disulfide. ACS Nano 2017, 11, 1755-1761.

[45] Huang, B.; Deng, H.; Lee, H.; Yoon, M.; Sumpter, B. G.; Liu, F.; Smith, S. C.; Wei, S. Exceptional Optoelectronic Properties of Hydrogenated Bilayer Silicene. Phys. Rev. X 2014, 4, 021029. 


\section{Experimental Methods}

\subsection{Scanning tunneling microscopy}

The scanning tunneling microscopy (STM) was invented by G. Binnig and W. Rohrer at the IBM Research Laboratory in 1982, ${ }^{[1,2]}$. The inventers of the STM received the Nobel Prize in Physics in 1986. It has been widely used as an instrument for real space analysis in surface science and related research fields. The STM can provide a lateral resolution of $0.1 \mathrm{~nm}$ and a vertical resolution of $0.01 \mathrm{~nm}$. Since STM relies on quantum mechanical tunneling the technique only allows to study conducting substrates, such as metals and semiconductors. Not only the structural, but also the electronic properties can be obtained by STM measurements with an unprecedented spatial resolution. It has to be emphasized here that an STM image cannot just be interpreted as a topographic map because the tunneling current is influenced by local density of states at the surface.

\subsubsection{The basic principle}

The basic idea of an STM is to bring a sharp metallic tip in close proximity (a few Å) to a sample, and apply a small bias between tip and substrate such that a small tunnel current (0.01$50 \mathrm{nA}$ ) starts to flow from the tip to the sample or vice versa. Figure 2.1a displays a schematic diagram of an STM. There is no physical contact between the tip and the substrate and electrons simply tunnel through a thin vacuum barrier with a width of about $1 \mathrm{~nm}$ and a barrier height of a few eVs.

(a)

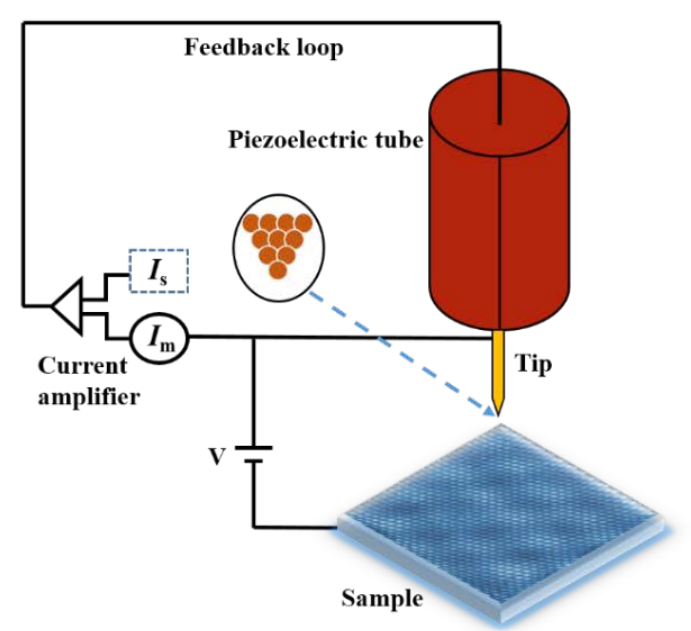

(b)

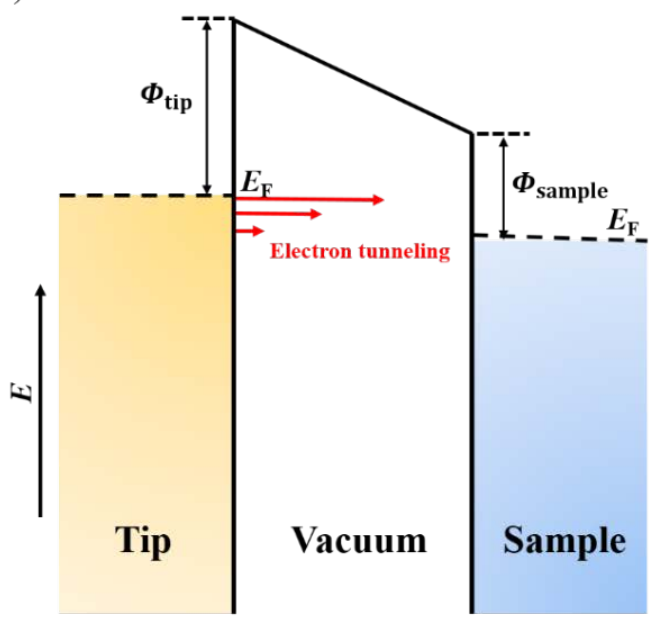

Figure 2.1: (a) Schematic diagram of an STM (b) Energy diagram of an STM tunnel junction. 
While the tip is scanned across the substrate the tunnel current is kept constant by continuously adjusting the tip-substrate distance using a piezo (see Figure 2.1a). Let us consider an electron wave with wave function $\Psi_{(\mathrm{x})}$ and energy $\mathrm{E}$ that encounters a potential barrier of height $\Phi$. The wave function of the electron satisfies the Schrödinger equation,

$$
\frac{-\hbar^{2}}{2 m} \frac{\partial^{2} \Psi_{(\mathrm{x})}}{\partial x}=(E-\Phi) \Psi_{(\mathrm{x})},
$$

where $\hbar$ is the reduced Planck's constant, $\mathrm{m}$ is the mass of the electron and $\mathrm{x}$ the position. If the barrier is higher than the energy of the incoming electron the solution of the wave function is given by

$$
\Psi_{(\mathrm{x})}=\Psi_{(0)} e^{-\kappa x}
$$

here, $\kappa=\frac{\sqrt{2 m(\Phi-E)}}{\hbar}$ is the inverse decay length. Then the probability (W) of finding the electron just behind the barrier with a width $z$ is given by

$$
W_{(\mathrm{z})}=\left|\Psi_{(\mathrm{z})}\right|^{2}=\left|\Psi_{(0)}\right|^{2} e^{-2 \kappa z}
$$

For the small bias $V$, the tunneling current $I$ is proportional to the probability of electrons to tunnel through the barrier, as well as the number of electrons in the energy window between $E_{f}-e V$ and $E_{f}$,

$$
I \propto \sum_{E_{f}-e V}^{E_{f}}\left|\Psi_{(0)}\right|^{2} e^{-2 \kappa z}
$$

And by definition, summing the probability over an energy range can give the number of states available in this energy range per unit volume, for the energy $\varepsilon \rightarrow 0$, the local density of states $\rho(z, E)$ is given by

$$
\rho(z, E)=\frac{1}{\varepsilon} \sum_{E_{f}-\varepsilon}^{E_{f}}\left|\Psi_{(\mathrm{z})}\right|^{2}
$$

Using equations (4) and (5), the tunneling current can be written in terms of LDOS as,

$$
I \propto \mathrm{V} \rho\left(0, E_{f}\right) e^{-2 \kappa z} .
$$

\subsubsection{Scanning tunneling spectroscopy}

There are several spectroscopic modes in STM. Here we discuss only the most important modes. 
I(V) spectroscopy In this spectroscopic mode the feedback loop of the STM is disabled and the tunneling current, $I$, is measured as a function of sample bias, $V$. Since the feedback loop is disabled it basically means the tip-substrate distance is kept constant while the $I(V)$ trace is recorded. In order to reduce the effect to thermal drift $I(V)$ are usually recorded within a second.

$\boldsymbol{d I} / \boldsymbol{d} \boldsymbol{V}$ spectroscopy The differential conductance, $d I / d V$, is proportional to the local electronic density of states (LDOS). By measuring the tunneling current as a function of sample bias, i.e. $I(V)$, the LDOS or differential conductivity can be obtained by simply taking numerically differentiating the $I(V)$ curve. At negative sample bias, electrons tunnel from surface to tip, which allows to investigate the LDOS of the filled states, while at positive sample bias the electrons tunnel into the empty states of the sample allowing us to investigate LDOS of the empty states of the surface.

Alternatively the differential conductivity can also be obtained by modulating the sample bias with a small sinusoidal voltage and subsequently use a lock-in amplifier to demodulate the tunnel current with the same frequency. The measured lock-in signal is proportional to the differential conductivity. Since in this method the feedback loop remains enabled at all times the differential conductivity can also be measured simultaneously with the standard topographic signal.

I(z) spectroscopy Current-distance spectroscopy is used to determine the barrier height of the tunnel junction. Here, the tunneling current is recorded as the STM tip is approached to or retracted from the surface. The barrier height between tip and surface is extracted by plotting the logarithm of the tunnel current versus the tip-surface distance. The slope of this curve gives the inverse decay length with is proportional to the square root of the barrier height.

\subsubsection{Omicron STM-1}

All the STM results are measured by a commercially available Omicron STM-1, which is a room temperature ultra-high vacuum STM. The base pressure in the STM chamber is about $3 \times 10^{-11}$ mbar. This system is mainly made up of three parts, a load-lock, a preparation chamber and an STM chamber. The load-lock is used to quickly transfer the samples from the air to the vacuum system or vice versa. In the preparation chamber we can clean the sample surface by $\mathrm{Ar}^{+}$ion sputtering and annealing. In addition, we can also deposit materials via physical vapor deposition. The setup of our Omicron STM-1 system is shown in Figure 2.2. 


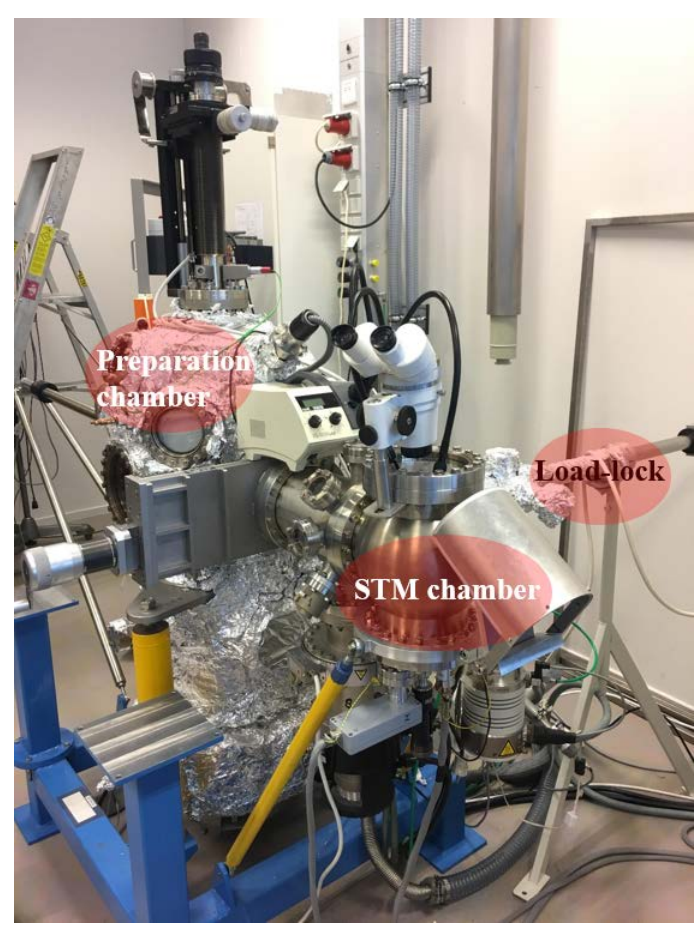

Figure 2.2: The STM-1 system.

\subsection{Conductive Atomic Force Microscopy}

Conductive atomic force microscopy is developed by Murrel and co-workers at the University of Cambridge in 1993, ${ }^{[3]}$. Conductive atomic force microscopy (C-AFM) is a very powerful tool to perform nano-scale research of the electrical properties of materials and devices. Unlike in the STM, the topography information in C-AFM is completely separated from the electrical signal. In order to obtain the electrical signal, a voltage is applied between the C-AFM tip and the sample. During the measurement, the cantilever is brought in contact with the sample, referred as the contact mode. The resulting current is recorded by using a highly sensitive amplifier as a current-to-voltage converter. This amplifier should be as close as possible to the tip in order to minimize noise. The two-dimensional currents map can be obtained together with the topographic image. Subsequently, the correlation between the topography and electrical properties of materials can be extracted. In addition, localized single point measurements of the current-voltage curves can also be obtained by the C-AFM. In this case, the cantilever is placed at the desired location on the sample surface and then the current signal is recorded while ramping the bias voltage. 


\section{Bibliography}

[1] Binnig, G.; Rohrer, H.; Gerber, C.; Weibel, E. Surface Studies by Scanning Tunneling Microscopy. Phys. Rev. Lett. 1982, 49, 57.

[2] Binnig, G.; Rohrer, H. Scanning Tunneling Microscopy- from Birth to Adolescence. Rev. Mod. Phys. 1987, 59, 615.

[3] Murrell, M. P.; Welland, M. E.; O’Shea, S. J.; Wong, T. M. H.; Barnes, J. R.; McKinnon, A. W.; Heyns, M.; Verhaverbeke, S. Spatially Resolved Electrical Measurements of $\mathrm{SiO}_{2}$ Gate Oxides Using Atomic Force Microscopy. Appl. Phys. Lett. 1993, 62, 786-788. 


\section{Moiré band structure in twisted graphene}

\subsection{Introduction}

In $2010 \mathrm{Li}$ et al. ${ }^{[1]}$ used scanning tunneling microscopy and spectroscopy to analyze these Van Hove singularities in twisted graphene layers. For small twist angles these authors observed two well-defined Van Hove singularities, one located just above the Fermi level and the other one located just below the Fermi level. The experimentally determined energy separation between these two Van Hove singularities nicely agrees with tight-binding calculations, provided that reasonable assumptions for the hopping integrals are made ${ }^{[1]}$. In addition, the authors pointed out that the two Van Hove singularities can become asymmetric (in position with respect to the Fermi level and amplitude) due to the presence of an interlayer bias. This interlayer bias is caused by the potential that is applied across the scanning tunneling microscopy junction. In the scanning tunneling microscopy data by Yin et al. ${ }^{[2]}$ a similar asymmetry and shift was found and discussed. Yan et al. ${ }^{[3]}$ studied the angle-dependent Van Hove singularities and found, in contrast to predictions by band structure calculations, that the Fermi velocity is very comparable to the Fermi velocity of monolayer graphene. In a follow-up study Yan et al. ${ }^{[4]}$ showed the breakdown of Van Hove singularities beyond a twist angle of about $3.5^{\circ}$, indicating that the continuum models are no longer applicable at these relatively large twist angles. Yin et al. ${ }^{[5]}$ showed that there is a magic twist angle of $1.11^{\circ}$ at which the two Van Hove singularities merge together and form a well-defined peak at the charge neutrality point. In addition to this strong peak at the charge neutrality point, these authors also found a set of regularly spaced peaks. These regularly spaced peaks are confined electronic states in the twisted bilayer graphene. The energy spacing of $70 \mathrm{meV}\left(=v_{F} / D\right)$ agrees well with the periodicity of the moiré pattern. In another study Yin et al. ${ }^{[2]}$ demonstrated that tilt grain boundaries can severely affect the structural and electronic properties of graphene multilayers. They also pointed out that tilt grain boundaries in trilayer graphene can result in the coexistence of massless Dirac fermions and massive chiral fermions. Wong et al. ${ }^{[6]}$ performed local spectroscopy on gate-tunable twisted bilayer graphene. The twisted graphene bilayer was positioned on a hexagonal boron nitride substrate. Wong et al. ${ }^{[6]}$ found, besides the coexistence of moiré patterns and moiré super-superlattices, also a very rich and interesting electronic structure. Despite the fact that the electronic structure of the twisted bilayer graphene has been extensively studied ${ }^{[1-15]}$, the spatial variation of the electronic structure within the unit cell of the moiré pattern did not receive a lot of attention yet. 
Here, in this chapter, we have studied the spatial variation of the electronic structure of twisted graphene on highly oriented pyrolytic graphite substrates. In the twisted graphene, we found the development of two Van Hove singularities in the density of states, which is in agreement with the previous studies. Spatial maps of the differential conductivity of the moiré pattern near the Fermi level reveal a honeycomb structure that is comprised of two inequivalent interpenetrating hexagonal sublattices. At large energies, i.e., $\left|E-E_{F}\right|>0.3 \mathrm{eV}$, the difference in the density of states of the two hexagonal sublattices fades away. Here we show that the inequivalence of these two sublattices can be understood if one takes into account a lowering of the symmetry due to the presence of the substrate. We will model this by introducing a third graphene layer. The fact that the spatial variation of the differential conductivity fades away at high energies hints to an electronic instability.

\subsection{Experimental and calculation details}

The experiments were performed with an ultrahigh vacuum (UHV) scanning tunneling microscope (Omicron). The base pressure of the UHV system is $3 \times 10^{-11}$ mbar. Before insertion of the ZYA quality highly oriented pyrolytic graphite (HOPG) substrates into the load lock of the UHV system we had removed several graphene layers via mechanical exfoliation using the Scotch-tape method. In order to remove any residual water from the HOPG surfaces, we had baked the load-lock system for $24 \mathrm{~h}$ at a temperature of $120^{\circ} \mathrm{C}$. After cooling down, the samples were transferred to the main chamber and subsequently inserted into the scanning tunneling microscope for imaging.

The scanning tunneling microscopy images were recorded in the constant current mode. Scanning tunneling spectroscopy spectra were recorded in two ways. In the first method we recorded current-voltage $(I(V))$ curves at many locations of the surface with the feedback loop of the scanning tunneling microscope disabled. The $d I / d V$ spectra were obtained by numerical differentiation of the I-V traces. In the second method a small sinusoidal voltage with a small amplitude of a few $\mathrm{mV}$ and a frequency of $1.9 \mathrm{kHz}$ was added to the bias voltage. A lock-in amplifier was used to record the $d I / d V$ signal.

The theoretical calculations for twisted graphene had been performed within the framework of the Slater-Koster tight-binding model, in which we took into account the intralayer and interlayer hoppings between the $p_{z}$ orbitals. The nearest intralayer hoppings in all layers are fixed as $t=3 \mathrm{eV}$, and the interlayer hopping between two sites in different layers is given by 


$$
t_{\perp}=\cos ^{2} \alpha V_{\sigma}+\sin ^{2} \alpha V_{\pi}
$$

where the orbital overlap is modeled as function of the angle $\alpha$ between the line connecting the two sites and the normal of the graphene plane, while $V_{\sigma}$ and $V_{\pi}$ are Slater-Koster integrals depending on the distance between the two sites. Both $V_{\sigma}$ and $V_{\pi}$ decay rapidly when the distance between the two sites is larger than the lattice parameter $a_{0}=2.46 \AA$, and the contribution of $V_{\pi}$ is negligible in the interlayer hoppings in multilayer graphene ${ }^{[14,15]}$. Here we use $0.24 \mathrm{eV}$ as the maximum value of $V_{\sigma}$ (for two sites with A-A stacking, the same value as used in Ref. [1]), and consider the screening effects following the environment-dependent tightbinding model introduced in Eq. (1) of Ref. [15]. The values of seven parameters fitted for the screening in multilayer graphene are taken from Ref. [15] as $\alpha_{1}=6.175, \alpha_{2}=0.762, \alpha_{3}=0.179$, $\alpha_{4}=1.411, \beta_{1}=6.811, \beta_{2}=0.01$, and $\beta_{3}=19.176$. All the neighboring pairs within a maximum in-plane distance of $2 \AA$ are included in the Hamiltonian. The electronic properties such as the density of states and quasi-eigenstates, which have the real-space profiles comparable to the experimental STM results, are calculated by using the tight-binding propagation method $(\mathrm{TBPM})^{[16,17]}$. TBPM has the advantages that the physical properties are extracted directly from the time evolution of the wave function, without any diagonalization of the Hamiltonian matrix.

\subsection{Spatial resolved electronic structure of twisted graphene}

When two layers of graphene are stacked on top of each other the electronic structure alters substantially. The low energy electronic band structure of bilayer graphene depends on how the two graphene layers are stacked ${ }^{[7]}$. The most common stacking is the so-called AB or Bernal stacking. The atoms of one of the hexagonal sublattices of the top layer (A1) are located on top of the atoms of one of the sublattices of the bottom layer (B2). The other atoms (B1 and A2) do not lie directly below or above an atom of the other layer. Highly oriented pyrolytic graphite is often stacked in the Bernal configuration. Commensurably twisted bilayer graphene can result in two different moiré lattice types ${ }^{[18]}$. The first type has a simple two-dimensional hexagonal superlattice, which is similar to the AB-stacked (Bernal) lattice. The other type has a twodimensional honeycomb superlattice comprising two equivalent hexagonal superlattices, and is similar to the AA-type stacked lattice. The honeycomb cases can be generated by twisting the two layers relative to one another over special angles $\theta$ obtained from the relation ${ }^{[18,19]}$

$$
\cos (\theta)=\frac{2 n^{2}+2 n m-m^{2}}{2\left(n^{2}+n m+m^{2}\right)},
$$


in which the integers $n$ and $m$ have no common divisors, and $n-m$ is not an integer multiple of $3^{[1]}$. The superlattice vectors are then given by $A_{1}=n a_{1}+m a_{2}$, respectively, $A_{2}=-m a_{1}+(n+$ m) $a_{2}$ with a supercell size factor $N=n^{2}+n m+m^{2}$ larger than in graphene ${ }^{[20,21]}$. The simple hexagonal lattice type can be obtained from the same relations by twisting over the special angles $\theta+\pi$. Figure 3.1 shows the scanning tunneling microscopy images of twisted graphene layers on the highly oriented pyrolytic graphite surface recorded at room temperature. Different twisted angles $\theta$ are extracted according to the periodicity $\lambda$ of the moiré pattern,

$$
\lambda=a_{0} / 2 \sin \left(\frac{\theta}{2}\right)
$$

where $a_{0}=0.246 \mathrm{~nm}$ is the lattice constant of graphene.
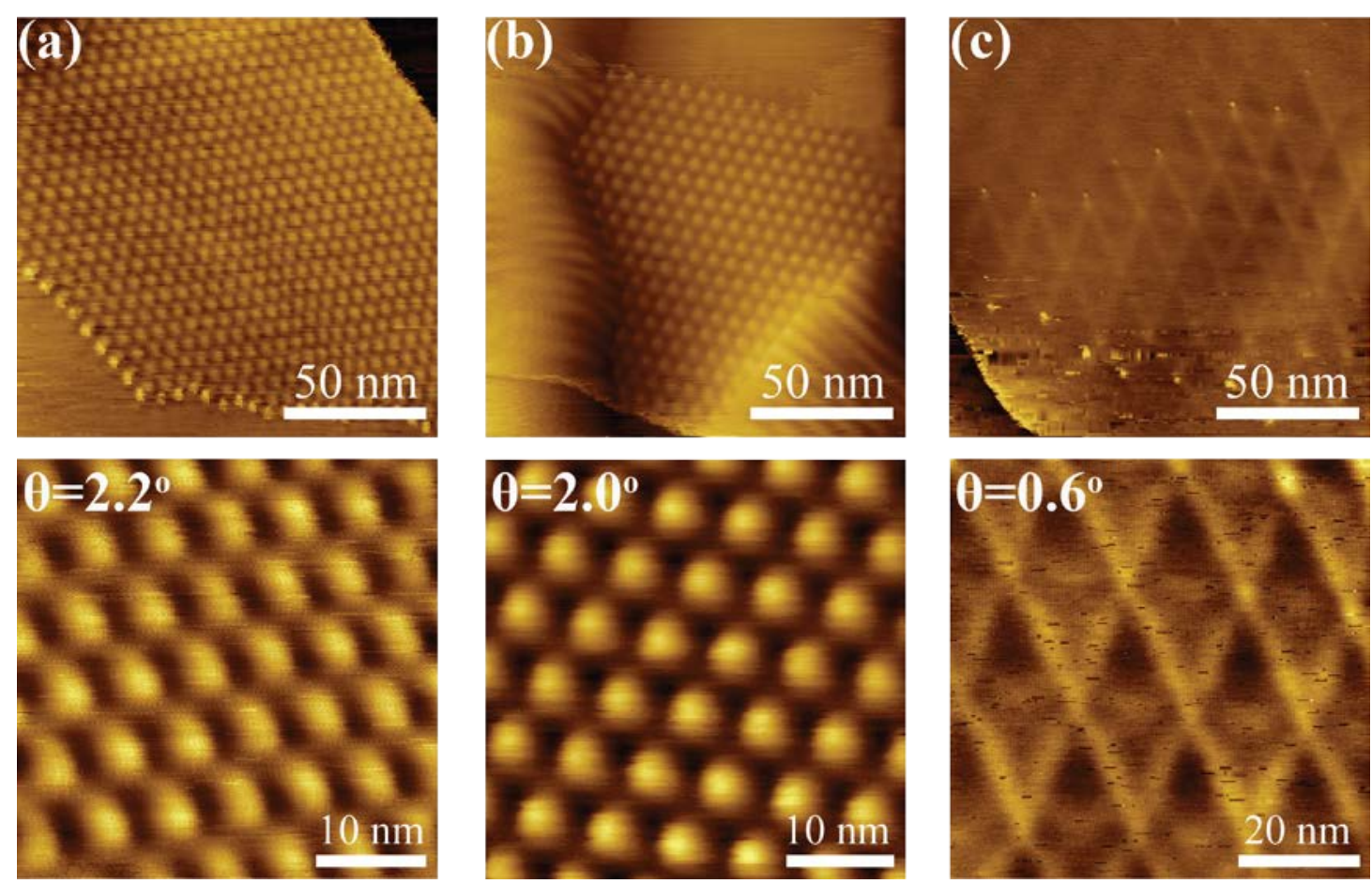

Figure 3.1: Scanning tunneling microscopy images of twisted graphene with different twisted angles, (a) $2.2^{\circ}$; (b) $2.0^{\circ}$; (c) $0.6^{\circ}$.

The electronic structure of this moiré pattern is characterized by a set of two Dirac cones that are located close to each other in reciprocal space. The crossing of these two Dirac cones results into two Van Hove singularities (vHS). As shown in Figure 3.2, as the AA stacking bilayer graphene is twisted by $\theta$, the Brillouin zones of the graphene layers are equally rotated by $\theta$. Thus, the Dirac cones of each layer are now centered in different points of the reciprocal space $K_{1}$ and $K_{2}$. The cones merge into two saddle points at energies $\pm E_{\mathrm{vHS}}$ from the Dirac point, leading to vHS which generate peaks in the DOS. Here the energy difference $\Delta E_{\mathrm{vHS}}$ follows ${ }^{[1,}$ 7]: 


$$
\Delta E_{\mathrm{vHS}}=2 \hbar v_{F} \Gamma K \sin \left(\frac{\theta}{2}\right)-2 t_{\theta},
$$

where $v_{F}$ is the Fermi velocity for monolayer graphene, $\Gamma K=1.703 \AA^{-1}$ is the wave vector of the Dirac point in monolayer graphene, and $t_{\theta}$ is the modulus of the amplitude of the main Fourier components of the interlayer potential.

(a)

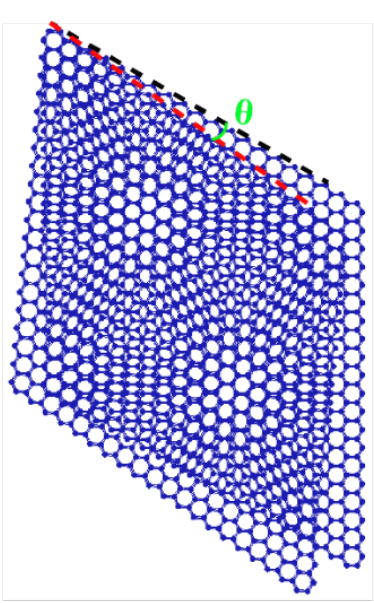

(b)

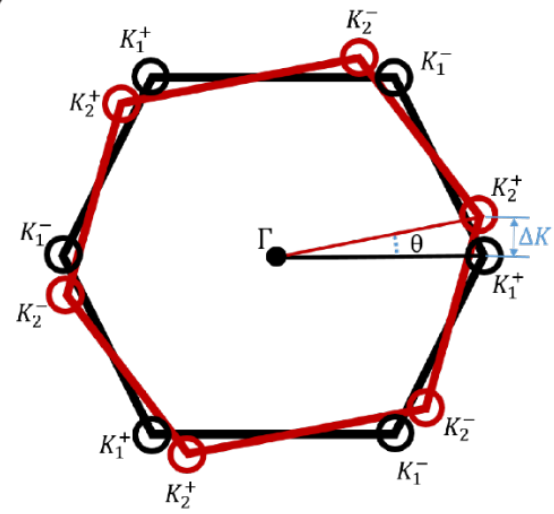

(c)

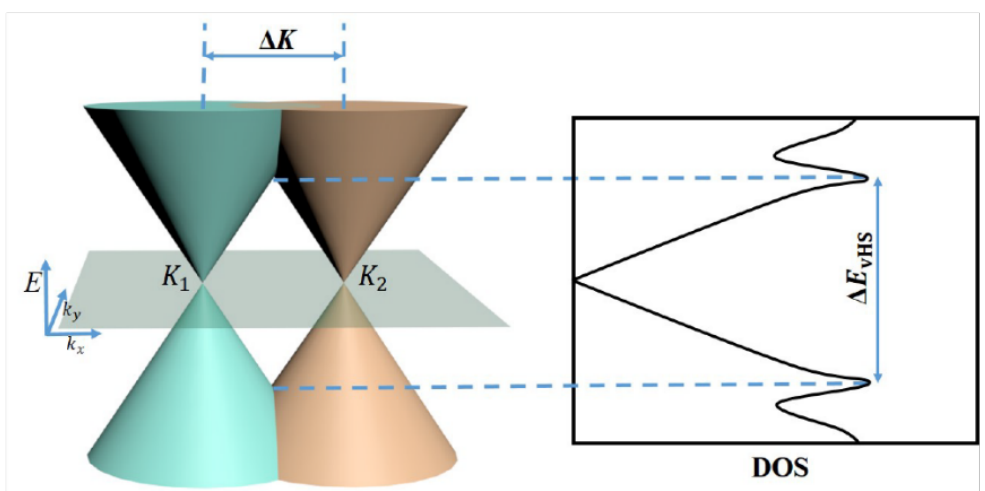

Figure 3.2: Origin of the Van Hove singularities in twisted graphene layer (a) Illustration of a moiré pattern arising from a twisted angle $\theta$. (b) Corresponding rotation in reciprocal space. (c) Emergence of vHS.

In Figure 3.3a, a scanning tunneling microscopy image of a twisted graphene with a twist angle of $2.0^{\circ}$ is displayed. The image is recorded at room temperature and the moiré pattern has a periodicity of $7.0 \mathrm{~nm}$. The differential conductivity $d I / d V$, which is proportional to the density of states for small biases, is depicted in Figure 3.3b. The $d I / d V$ curves recorded at a $60 \times 60$ grid of the surface displayed in the inset of Figure 3.3a. Two well-defined peaks are found at energies of $-110 \mathrm{meV}$ and $15 \mathrm{meV}$ with respect to the Fermi level, respectively. These two peaks are Van Hove singularities. At the high regions of the moiré pattern the peaks have a higher intensity as compared to the lower regions of the moiré pattern. The energy separation, 
relative strength, and asymmetry are in good agreement with Ref. [1]. The $d I / d V$ spectra in Figure 3.3b are recorded at room temperature and therefore these peaks are much broader than the peaks that are reported in Ref.[1], which are taken at $4 \mathrm{~K}$.
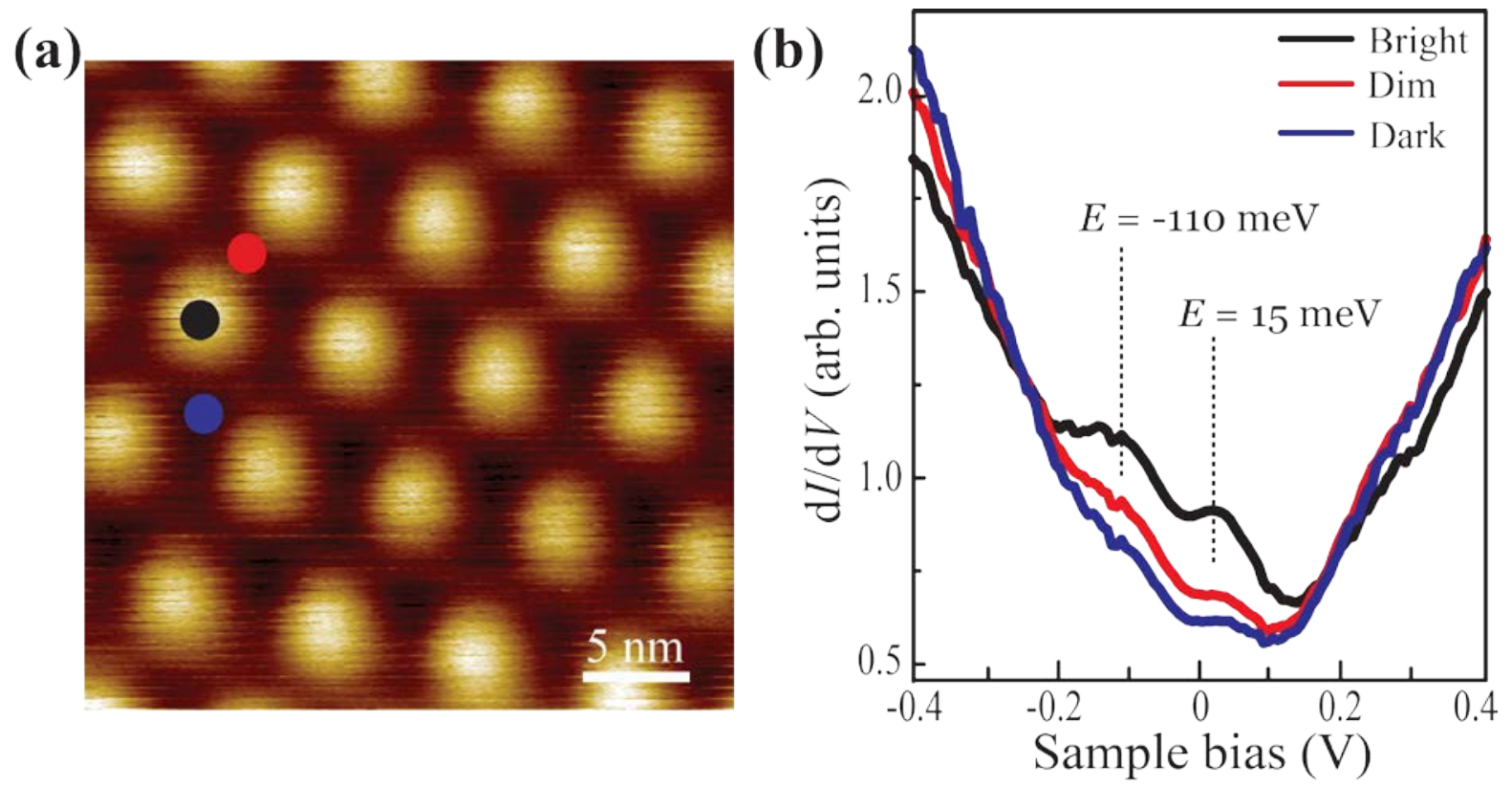

Figure 3.3: (a) Scanning tunneling microscopy image of twisted graphene. (b) Differential conductivity recorded at different locations (bright, dim and dark).

In order to understand the experimental observations shown in Figure 3.3b, we have performed theoretical calculations of the density of states by using the Slater-Koster tight-binding model for rotated bilayer and trilayer graphene, respectively. The numerical results of the integrated density of states are plotted in Figure 3.4.

It is clear that although the two Van Hove singularities are always present when there is a rotated graphene layer, one has to take into account the third layer in order to reproduce the significant electron-hole asymmetry and the finite density of the states in the vicinity of the Fermi level. The electron-hole asymmetry is enhanced if the interlayer hoppings between the top and the third layer are also included. Furthermore, by turning on the direct interactions between the top and the third layer, the whole energy spectrum is shifted to the hole direction, similar to the experimental observations. Here we want to emphasize that for a heterostructure consisting of a rotated graphene layer on top of graphite, it is not sufficient to only consider a rotated bilayer graphene in the theoretical studies. The influence of the third layer, either indirectly via the hoppings to the middle layer, or directly via the interactions to the top layer, is not negligible. 
It is therefore necessary to consider at least three layers in the calculations of the electronic structure and physical properties.

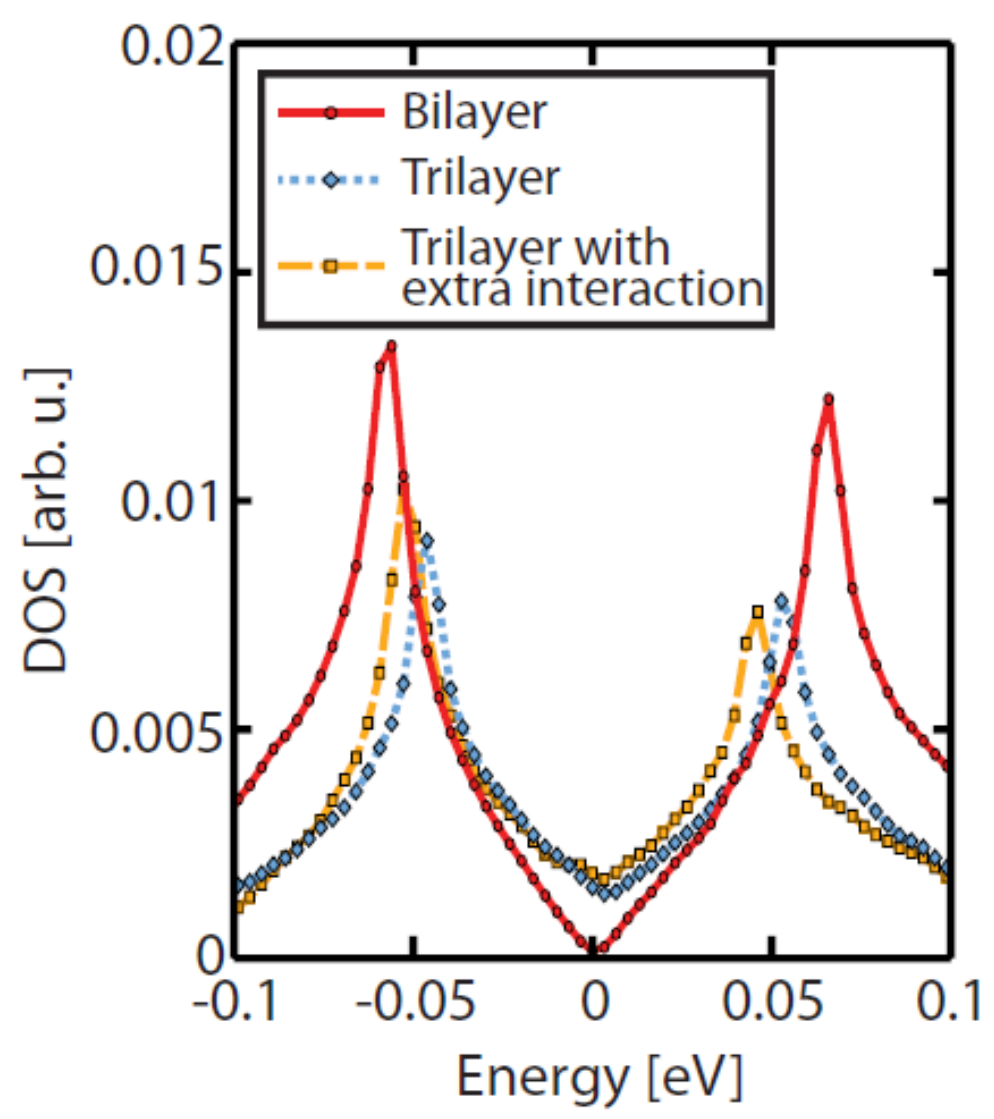

Figure 3.4: (b) Calculated total density of states for rotated bilayer and trilayer graphene $(\theta=$ $2.0^{\circ}$, for trilayer graphene with an extra interaction interlayer hoppings between the top and bottom layers with a maximum value of $0.1 \mathrm{eV}$ are included (for two sites on A-A stacking).

In the two middle panels of Figure 3.5, spatial maps of the differential conductivity are shown at various energies. The moiré pattern is present in the differential conductivity maps that are recorded near the Fermi level, but the structure fades away at larger energies. Also this observation is consistent with Ref. [1], albeit the sample bias range where we observe the moiré pattern in the $d I / d V$ signal is substantially larger.

With aim of understanding this strong energy dependence of the differential conductivity maps we have performed tight-binding calculations of a quasi-eigenstate, which is a superposition state of all degenerate eigenstates at a given energy ${ }^{[15]}$. The real-space distribution of the wave 
amplitude in a quasi-eigenstate corresponds to the local density of states measured in the scanning tunneling microscopy experiments ${ }^{[16]}$. In the left and right panels of Figure 3.5 we

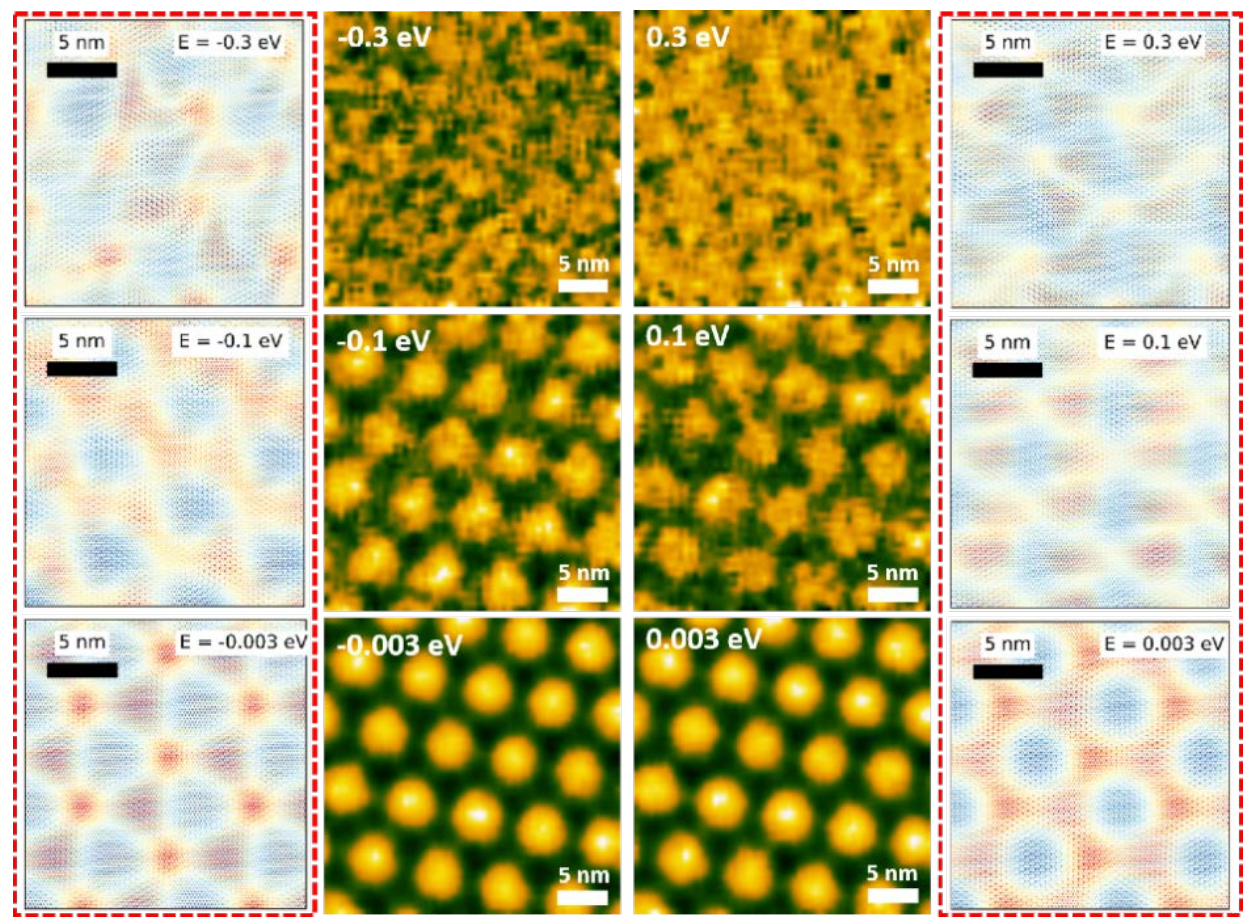

Figure 3.5: Middle panels: Spatial map of the differential conductivity at different bias voltages. The large bright spots in the $d I / d V$ maps correspond to the higher parts of the moiré pattern [see Figure 3.3a]. Left and right panels (in red dashed box): The real-space amplitude (logarithmic scale) of the calculated quasieigenstates for trilayer graphene with twisted top layer $\left(\theta=2.0^{\circ}\right)$. The results are obtained by averaging over 24 initial states to mimic the randomness introduced by the initial state. In each figure, blue and red correspond to the maximal and minimal intensity, respectively. For higher absolute energy this amplitude is lower.

show contour plots of several quasi-eigenstates for a layer of rotated graphene stacked on top of an AB-stacked bilayer graphene. Our theoretical calculations of this heterostructure consisting of three graphene layers show exactly the same tendency as the experimental data, i.e., the hexagonal structure in the density states is only present near the Fermi level and fades away at higher energies.

The fact that the differential conductivity only exhibits a density modulation near the Van Hove singularities is reminiscent of a charge density wave. One of the hallmarks of a charge density wave is that the electron density and the lattice positions are coupled. Charge density waves 
may be generated by an exchange-driven instability of a metallic Fermi surface (Fermi nesting), or by a lattice-dynamical instability leading to a static periodic lattice distortion. It is important to point out here that a periodic potential in a Dirac system will not result in the opening of a band gap, but rather in the creation of new Dirac points and Van Hove singularities ${ }^{[22,23]}$. The concept of charge density of waves needs therefore to be revisited for Dirac systems. The energy dependent electron density modulation that we measured for twisted graphene can be fully explained by tight-binding calculations. Since electron-phonon coupling is not included in these tight-binding calculations it remains to be seen whether we are dealing here with a charge density wave.

In Figure 3.6a, a high resolution spatial map of the differential conductivity of the strongest Van Hove singularity, which is located at $-110 \mathrm{meV}$, is shown. This spatial map is recorded with a lock-in amplifier (modulation voltage $20 \mathrm{mV}$ and frequency $1.9 \mathrm{kHz}$ ). The spatial $d I / d V$ map exhibits atomic resolution. Even the periodicity of the top graphene layer with a lattice constant of $0.246 \mathrm{~nm}$ is visible. For the sake of clarify we have inverted the color scale in Figure 3.6a, so dark regions refer to a high $d I / d V$ signal, whereas bright spots refer to a low $d I / d V$ signal. The honeycomb structure consists of two interpenetrating hexagonal sublattices. One hexagonal sublattice displays a substantially higher $d I / d V$ signal than the other hexagonal sublattice. The occurrence of these two hexagonal sublattices can be understood if one takes into account a third graphene layer that breaks the symmetry of a twisted bilayer graphene. The dominant stacking arrangement of HOPG is the Bernal (AB) stacking. Consequently, half of
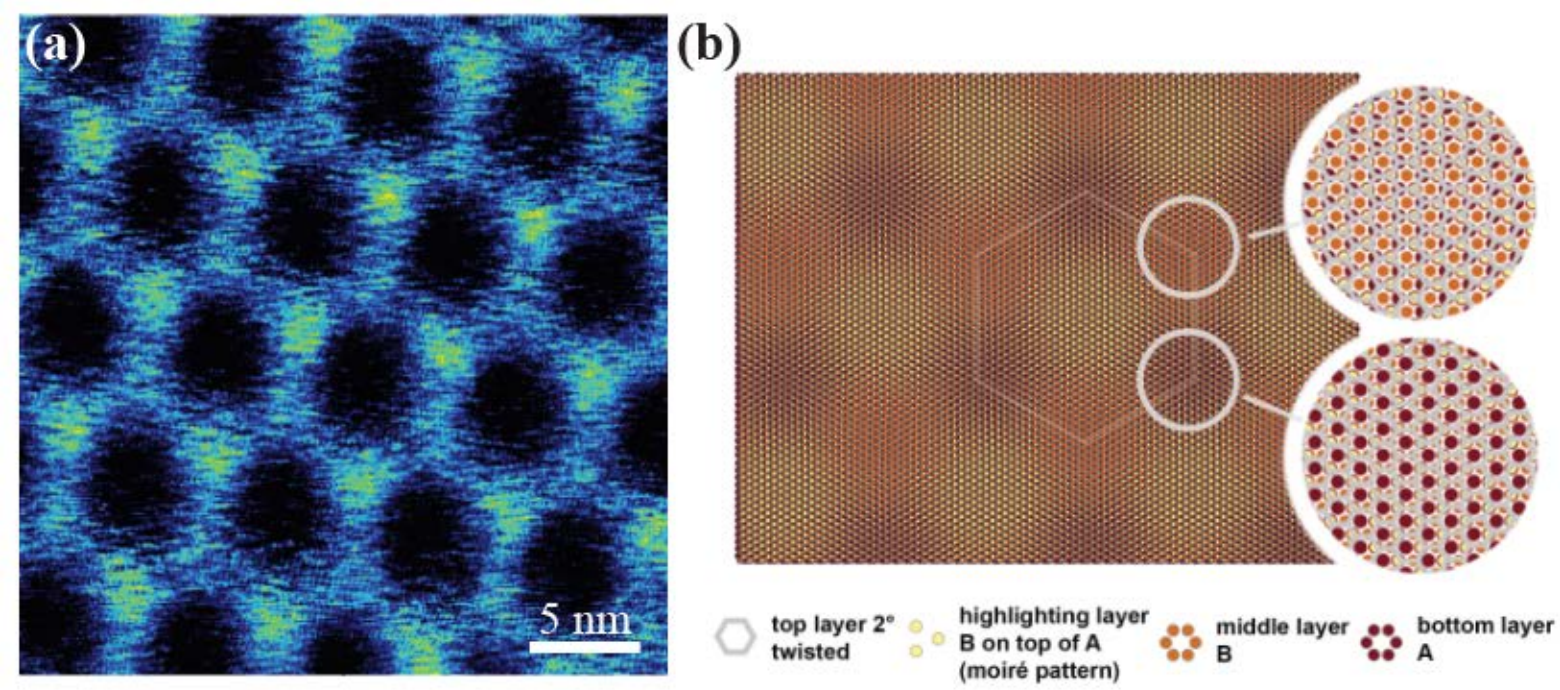

Figure 3.6: (a) Spatial map of the differential conductivity. (b) Structural model of trilayer graphene. 
the carbon atoms of the second graphene layer are located on top of a carbon atom of the bottom layer, whereas the other half of the second layer carbon atoms do not have a carbon atom underneath them. In Figure 3.6b the schematic diagram of the trilayer graphene is depicted: the two bottom graphene layers are AB stacked, whereas the top graphene layer is twisted by $2.0^{\circ}$ with respect to the second graphene layer. The honeycomb lattice of the moiré pattern in Figure $3.6 \mathrm{~b}$ is composed of two interpenetrating hexagonal sublattices. The highest $d I / d V$ signal is observed if the atoms in the second layer have atoms directly underneath them.

\subsection{Conclusions}

Spatially resolved scanning tunneling spectroscopy measurements of twisted graphene reveal a hitherto unnoticed variation of the density of states within the unit cell of the moiré pattern. A honeycomb pattern is found that is comprised of two inequivalent hexagonal sublattices. The symmetry of the honeycomb lattice of the moiré pattern is broken by a third graphene layer that is stacked in a Bernal configuration with respect to the second graphene layer. Our experimental findings are in excellent agreement with tight-binding calculations. 


\section{Bibliography}

[1] Li, G.; Luican, A.; Lopes dos Santos, J. M. B.; Castro Neto, A. H.; Reina, A.; Kong, J.; Andrei, E. Y. Observation of Van Hove Singularities in Twisted Graphene Layers. Nat. Phys. 2010, 6 (2), 109-113.

[2] Yin, L.-J.; Qiao, J.-B.; Wang, W.-X.; Chu, Z.-D.; Zhang, K. F.; Dou, R.-F.; Gao, C. L.; Jia, J.-F.; Nie, J.-C.; He, L. Tuning Structures and Electronic Spectra of Graphene Layers with Tilt Grain Boundaries. Phys. Rev. B 2014, 89 (20), 205410.

[3] Yan, W.; Liu, M.; Dou, R.-F.; Meng, L.; Feng, L.; Chu, Z.-D.; Zhang, Y.; Liu, Z.; Nie, J.C.; He, L. Angle-Dependent van Hove Singularities in a Slightly Twisted Graphene Bilayer. Phys. Rev. Lett. 2012, 109 (12), 126801.

[4] Yan, W.; Meng, L.; Liu, M.; Qiao, J.-B.; Chu, Z.-D.; Dou, R.-F.; Liu, Z.; Nie, J.-C.; Naugle, D. G.; He, L. Angle-Dependent van Hove Singularities and Their Breakdown in Twisted Graphene Bilayers. Phys. Rev. B 2014, 90 (11), 115402.

[5] Yin, L.-J.; Qiao, J.-B.; Zuo, W.-J.; Li, W.-T.; He, L. Experimental Evidence for NonAbelian Gauge Potentials in Twisted Graphene Bilayers. Phys. Rev. B 2015, 92 (8), 081406.

[6] Wong, D.; Wang, Y.; Jung, J.; Pezzini, S.; DaSilva, A. M.; Tsai, H.-Z.; Jung, H. S.; Khajeh, R.; Kim, Y.; Lee, J.; et al. Local Spectroscopy of Moirl'e-Induced Electronic Structure in Gate-Tunable Twisted Bilayer Graphene. Phys. Rev. B 2015, 92 (15), 155409.

[7] Lopes dos Santos, J. M. B.; Peres, N. M. R.; Castro Neto, A. H. Graphene Bilayer with a Twist: Electronic Structure. Phys. Rev. Lett. 2007, 99 (25), 256802.

[8] Ohta, T.; Bostwick, A.; Seyller, T.; Horn, K.; Rotenberg, E. Controlling the Electronic Structure of Bilayer Graphene. Science 2006, 313 (5789), 951-954.

[9] Castro, E. V.; Novoselov, K. S.; Morozov, S. V.; Peres, N. M. R.; dos Santos, J. M. B. L.; Nilsson, J.; Guinea, F.; Geim, A. K.; Neto, A. H. C. Biased Bilayer Graphene: 
Semiconductor with a Gap Tunable by the Electric Field Effect. Phys. Rev. Lett. 2007, 99 (21), 216802.

[10] Li, G.; Luican, A.; Andrei, E. Y. Scanning Tunneling Spectroscopy of Graphene on Graphite. Phys. Rev. Lett. 2009, 102 (17), 176804.

[11] Trambly de Laissardière, G.; Mayou, D.; Magaud, L. Localization of Dirac Electrons in Rotated Graphene Bilayers. Nano Lett. 2010, 10 (3), 804-808.

[12] Luican, A.; Li, G.; Reina, A.; Kong, J.; Nair, R. R.; Novoselov, K. S.; Geim, A. K.; Andrei, E. Y. Single-Layer Behavior and Its Breakdown in Twisted Graphene Layers. Phys. Rev. Lett. 2011, 106 (12), 126802.

[13] Brihuega, I.; Mallet, P.; González-Herrero, H.; Trambly de Laissardière, G.; Ugeda, M. M.; Magaud, L.; Gómez-Rodríguez, J. M.; Ynduráin, F.; Veuillen, J.-Y. Unraveling the Intrinsic and Robust Nature of van Hove Singularities in Twisted Bilayer Graphene by Scanning Tunneling Microscopy and Theoretical Analysis. Phys. Rev. Lett. 2012, 109 (19), 196802.

[14] Sboychakov, A. O.; Rakhmanov, A. L.; Rozhkov, A. V.; Nori, F. Electronic Spectrum of Twisted Bilayer Graphene. Phys. Rev. B 2015, 92 (7), 075402.

[15] Tang, M. S.; Wang, C. Z.; Chan, C. T.; Ho, K. M. Environment-Dependent TightBinding Potential Model. Phys. Rev. B 1996, 53 (3), 979-982.

[16] Yuan, S.; De Raedt, H.; Katsnelson, M. I. Modeling Electronic Structure and Transport Properties of Graphene with Resonant Scattering Centers. Phys. Rev. B 2010, 82 (11), 115448

[17] Yuan, S.; Wehling, T. O.; Lichtenstein, A. I.; Katsnelson, M. I. Enhanced Screening in Chemically Functionalized Graphene. Phys. Rev. Lett. 2012, 109 (15), 156601.

[18] Yıldız, D.; Şen, H. Ş.; Gülseren, O.; Gürlü, O. Apparent Corrugation Variations in Moirl'e Patterns of Dislocated Graphene on Highly Oriented Pyrolytic Graphite and the 
Origin of the van Hove Singularities of the Moirl'e System. ArXiv150200869 Cond-Mat 2015.

[19] Wijk, M. M. van; Schuring, A.; Katsnelson, M. I.; Fasolino, A. Relaxation of Moiré Patterns for Slightly Misaligned Identical Lattices: Graphene on Graphite. 2D Mater. 2015, 2 (3), 034010.

[20] Albrecht, T. R.; Mizes, H. A.; Nogami, J.; Park, S.; Quate, C. F. Observation of Tilt Boundaries in Graphite by Scanning Tunneling Microscopy and Associated Multiple Tip Effects. Appl. Phys. Lett. 1988, 52 (5), 362-364.

[21] Kuwabara, M.; Clarke, D. R.; Smith, D. A. Anomalous Superperiodicity in Scanning Tunneling Microscope Images of Graphite. Appl. Phys. Lett. 1990, 56 (24), 2396-2398.

[22] Park, C.-H.; Yang, L.; Son, Y.-W.; Cohen, M. L.; Louie, S. G. Anisotropic Behaviours of Massless Dirac Fermions in Graphene under Periodic Potentials. Nat. Phys. 2008, 4 (3), 213-217.

[23] Park, C.-H.; Yang, L.; Son, Y.-W.; Cohen, M. L.; Louie, S. G. New Generation of Massless Dirac Fermions in Graphene under External Periodic Potentials. Phys. Rev. Lett. 2008, 101 (12), 126804. 


\section{Intercalation of silicon in transition metal dichalcogenides}

\subsection{Introduction}

Since the isolation of graphene by Novoselov and Geim, ${ }^{[1]}$ two-dimensional (2D) materials have received a lot of attention. The method of delaminating graphite down to a single layer is facilitated by the crystal structure of graphite, which consists of 2D layers that are weakly bonded to each other via van der Waals forces. ${ }^{[2]}$ Besides graphite, other $2 \mathrm{D}$ materials such as the transition metal dichalcogenides (TMDs) also consist of these weakly van der Waals bonded layers. The chemical composition of each of these TMDs is $\mathrm{MX}_{2}$, where $\mathrm{M}$ refers to a transition metal, e.g., molybdenum or tungsten, and X stands for a chalcogen such as sulfur or selenium. Each TMD layer consists of three sheets with hexagonal symmetry that are covalently bonded to each other. In each of these triple layers, one layer of transition metal atoms is sandwiched in between two layers of chalcogen atoms. Since the TMDs have a similar layered structure as graphite, it is not surprising that a renewed interest in the TMDs aroused when it was found possible to also exfoliate these materials to a single layer. ${ }^{[3]}$ As such, it was found that the physical properties of TMDs such as the band gap ${ }^{[4]}$ and electron-phonon coupling ${ }^{[5]}$ depend on the number of TMD triple layers.

Pristine and free-standing graphene is gapless, and therefore this material cannot be used as the key material for the field-effect based devices. Many scientists have, however, tried to open a band gap in pristine graphene by breaking the sub-lattice symmetry. ${ }^{[6,7]}$ So far, these attempts have failed or resulted in a strong degradation of the charge carrier mobilities. A more suitable 2D material for realizing a field effect transistor is silicene, the silicon analogue of graphene, which naturally already displays a broken sub-lattice symmetry. The charge carriers in silicene have been predicted to behave similar to the massless Dirac fermions of graphene. ${ }^{[8]}$ Unlike graphite and the TMDs, silicene does not occur in nature and therefore it has to be synthesized. Several research groups have shown that silicene can be grown on $\operatorname{Ag}(111) .{ }^{[9-12]}$ Silicene synthesized on $\mathrm{Ag}(111)$ even displays a linear dispersion relation; ${ }^{[11]}$ however, the exact origin of this linear energy band is still under debate. ${ }^{[13]}$ Unfortunately, silicene has a strong electronic coupling with the $\operatorname{Ag}(111)$ substrate and as a result loses its Dirac fermion characteristics. ${ }^{[14]}$ To protect the unique electronic properties of silicene, the electronic coupling with the substrate should be reduced as much as possible. TMDs like $\mathrm{WSe}_{2}$ and $\mathrm{MoS}_{2}$, which have no dangling bonds, are atomically flat over large areas, and have a band gap, seem to fit all the requirements to support the growth of silicene as the interaction with the $2 \mathrm{D}$ adlayer is only via very weak 
van der Waals forces. ${ }^{[15]}$ This weak interaction will help to preserve the important electronic properties near the Fermi level. The growth of silicon on $\mathrm{MoS}_{2}$ has already been studied. ${ }^{[16,17]}$ Another appealing two-dimensional material is germanene. ${ }^{[18-21]}$ Recently, the synthesis of germanene on $\mathrm{MoS}_{2}$ has been reported. ${ }^{[22]}$ The density of states of germanene on $\mathrm{MoS}_{2}$ exhibits a well-defined V-shape, which is one of the hallmarks of a two-dimensional Dirac material. ${ }^{[23]}$ In this chapter, we report on the growth of submonolayers of silicon on tungsten diselenide (WSe 2 ). Upon deposition of silicon, the atomically flat $\mathrm{WSe}_{2}$ surface converts into a surface with a hill-and-valley structure. Guided by our high-resolution scanning tunneling microscopy (STM) and spectroscopy (STS) measurements, we provide compelling evidence that silicon does not grow on top of $\mathrm{WSe}_{2}$ but intercalates between the $\mathrm{WSe}_{2}$ layers.

\subsection{Experimental details}

The experiments were carried out in a system equipped with a room temperature Omicron STM (STM-1). The base pressure in the system is below $3 \times 10^{-11}$ mbar. All the STM and STS measurements were conducted at room temperature. The synthetic WSe 2 samples purchased from HQ Graphene were cleaned by mechanical exfoliation in ambient conditions after which they were immediately mounted on a sample holder and inserted into the ultra-high vacuum system. Limited contamination is expected because the samples are non-reactive. Silicon was deposited on the WSe2 samples via the resistive heating of a small piece of a silicon wafer. In order to calibrate the silicon source, we deposited a fraction of a monolayer of silicon on a $\mathrm{Ge}(001)$ substrate at room temperature. Subsequently, the $\mathrm{Ge}(001)$ substrate was mildly annealed at a temperature $450-500 \mathrm{~K}$. After that, we determined the coverage of the epitaxial silicon islands.

\subsection{Growth of silicon on tungsten diselenide}

Before the deposition of silicon, the samples of freshly cleaved WSe 2 are characterized by the constant current topography STM images. In this STM mode, the tip-sample distance is adjusted as to keep a constant tunneling current while the extension of the z-piezo is recorded. The lattice constant of the hexagonal lattice of WSe 2 is $3.28 \AA .{ }^{[24]}$ Even at larger length scales, the mechanically cleaved samples of WSe $\mathrm{W}_{2}$ are atomically flat, as can be seen in the inset of Figure 4.1. Only a few electronic defects can be observed in an area of $80 \times 80 \mathrm{~nm}^{2}$. The average terrace size of $\mathrm{WSe}_{2}$ is very large, and step edges are only rarely encountered in STM scans. 
After the deposition of about a quarter of a monolayer of silicon on clean $\mathrm{WSe}_{2}$, the WSe surface displays more roughness in the STM topography scans than the bare surface, as can be

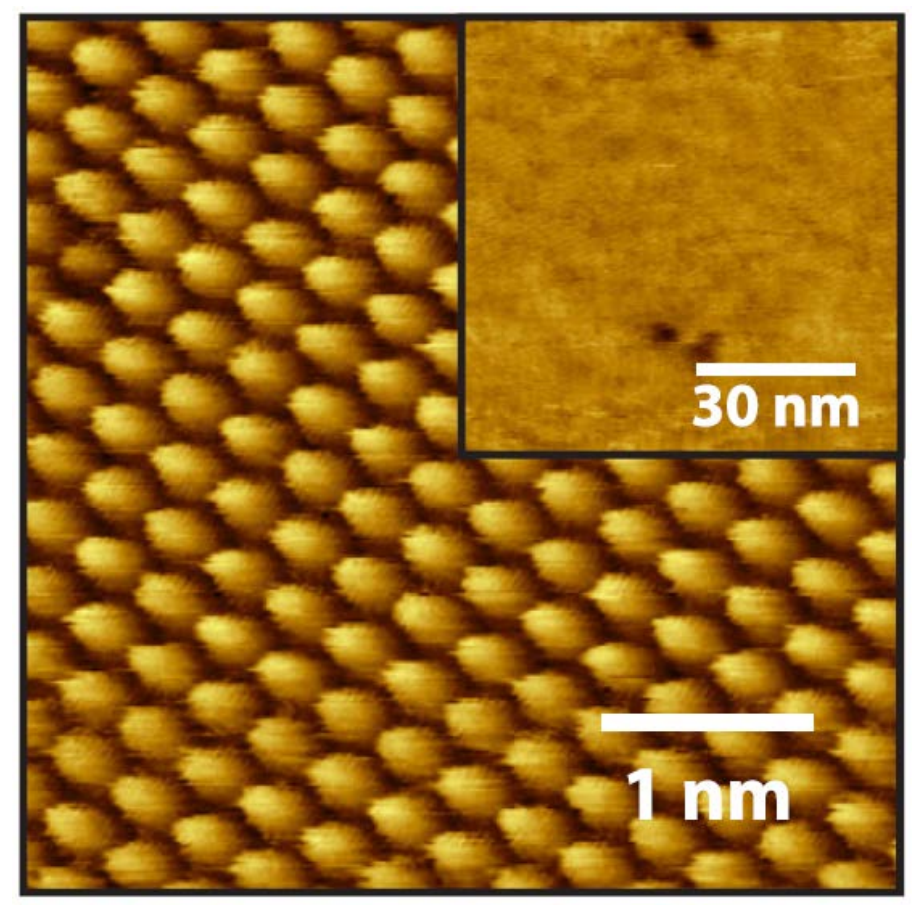

Figure 4.1: The clean atomically resolved surface of $\mathrm{WSe}_{2}(\mathrm{~V}=-0.6 \mathrm{~V}, \mathrm{I}=1.1 \mathrm{nA})$. Inset: Large area scan of WSe2 showing a few electronic defects.

seen in Figure 4.2a. A hill-and-valley structure can be observed in the topography scans, similar to the observations by Chiappe et al. ${ }^{[16]}$ and Molle et al. ${ }^{[17]}$ for the silicon on $\mathrm{MoS}_{2}$ system. Upon the deposition of more silicon, the surface becomes even rougher and after a few monolayers eventually 3D clusters are observed on the surface. It is not obvious at all that the growth does

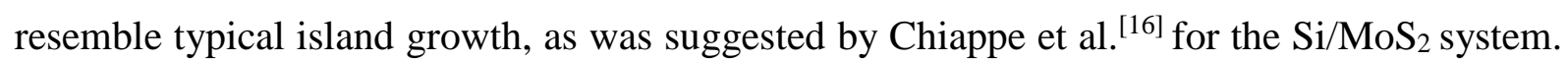
A firm argument against island growth is the fact that the lattice constant of the hill-and valley is exactly the same as the lattice constant of the bare $\mathrm{WSe}_{2}$ or $\mathrm{MoS}_{2}$ substrates. This is not compatible with the silicon island growth on top of $\mathrm{WSe}_{2}$ since the lattice constants of silicon or low-buckled silicene are substantially larger. ${ }^{[25]}$ Chiappe et al. ${ }^{[16]}$ interpreted the observed small lattice constant as the growth of strained high-buckled silicene. They argued that highbuckled silicene grows epitaxially on $\mathrm{MoS}_{2}$, i.e., the lattice constant of silicene adapts itself to the lattice constant of the $\mathrm{MoS}_{2}$ substrate. However, it should be pointed out that freestanding high buckled silicene is unstable because it has imaginary phonon modes in a large portion of the Brillouin zone. ${ }^{[26,27]}$ In addition, the interaction between the silicon adlayer and the substrate 
is governed by weak van der Waals forces and therefore the formation of a strained epitaxial silicon layer is highly unlikely. Actually, van der Waals heteroepitaxy has been demonstrated even for materials with a large lattice mismatch. ${ }^{[28]}$ Another strong argument against island growth is that the transition from a hill to a valley is very gradual, as can be seen in the line profile in Figure 4.2c. In the case of island growth on top of a substrate, one expects to encounter well-defined island edges, which show up as abrupt height variations in the constant current STM scans. The gradual transition from a hill to a valley does, however, support the idea of a buckled WSe 2 top layer.
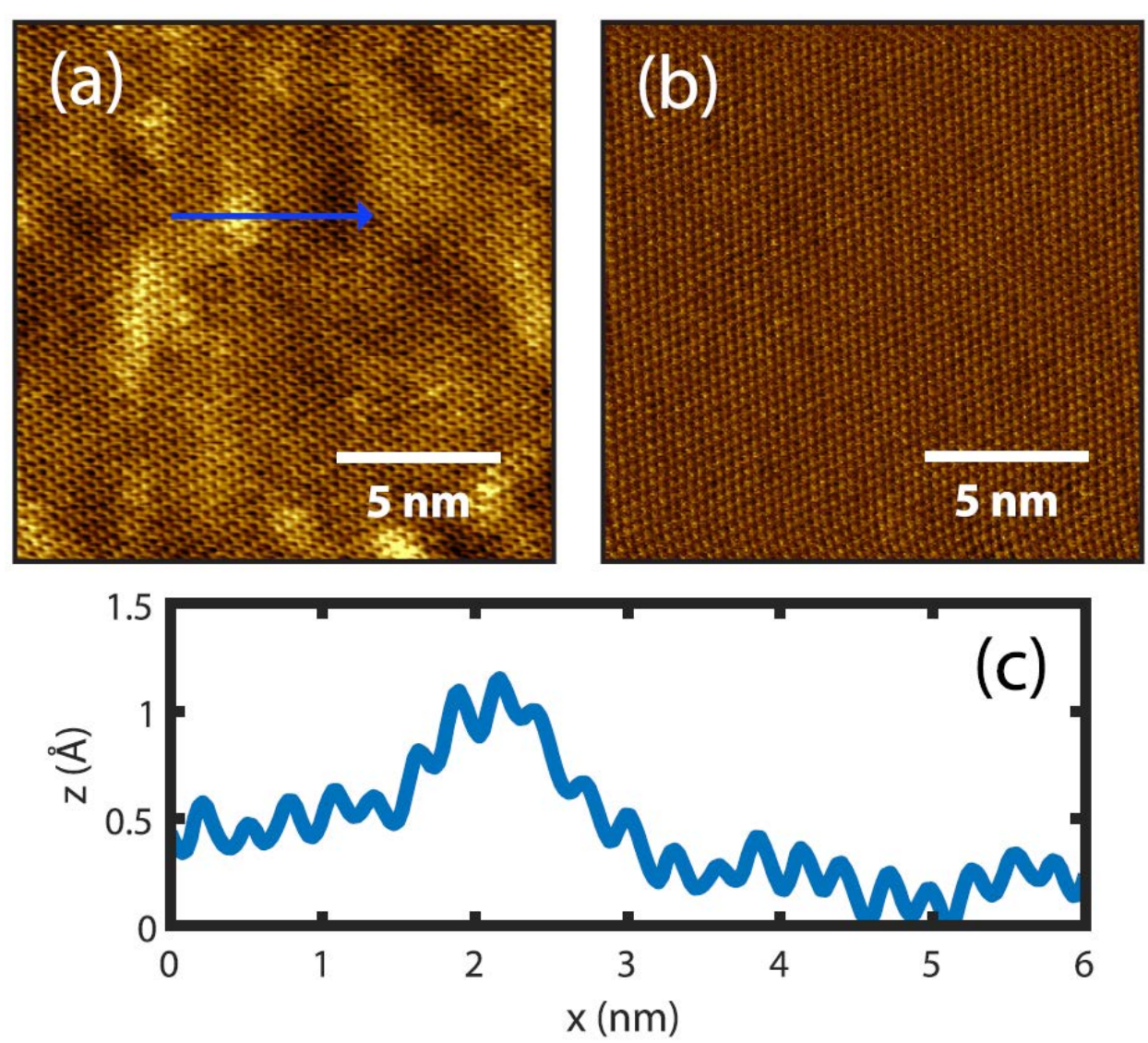

Figure 4.2: Constant current topography (a) and $d I / d z$ (b) maps of $\mathrm{WSe}_{2}$ after deposition of $0.25 \mathrm{ML}$ of silicon ( $\mathrm{V}=-0.8 \mathrm{~V}, \mathrm{I}=1.5 \mathrm{nA})$. The $d \mathrm{I} / \mathrm{dz}$ map is proportional to the local apparent barrier height. The maps are recorded simultaneously. The line profile indicated in (a) is displayed in panel (c).

At this point, it is still not clear what the hills and valleys in the topography scans exactly represent. This is because a constant current topography scan in the STM contains both topographic and electronic information. In order to determine the exact nature of the hill-andvalley structure, one needs to separate the topographic and electronic signals and obtain 
chemical sensitivity on the surface. In STM, this can be accomplished by operating the STM in the $d I / d z$ spectroscopy mode. The $d I / d z$ signal can be recorded simultaneously with the constant current topography. In the constant current mode, the z-piezo voltage is measured (the z-piezo voltage can be converted into a height $z_{0}$, which should be discriminated from, $z$, the tip-sample distance), whereas in the $d I / d z$ mode only the derivative of the current to $z$ is measured. The fact that the $d I / d z$ signal is not dependent on the local surface height variations can easily be understood within the framework of the Tersoff and Hamann approximation. ${ }^{[29]}$ The tunnel current $I$ is given by

$$
I=C \int_{0}^{e V} \rho_{t}(E-e V) \rho_{s}(E) T(V, E, z) d E,
$$

where $C$ is a proportionality constant, e the elementary charge, $V$ the applied voltage between tip and sample and $\rho_{t}$ and $\rho_{s}$ the density of states of the tip and sample, respectively. $T(V, E, z)$ is the tunneling probability and $E$ and $z$ are the electron energy and the tip-sample distance, respectively. The tunneling probability depends on the tip-sample distance $z$, but not $z_{0}$

$$
T(V, E, z)=\exp \left(-2 \frac{\sqrt{2 m}}{\hbar} z \sqrt{\varphi_{A}+\frac{e V}{2}-E}\right),
$$

with $m$ the electron mass and $\varphi_{A}$ the local apparent barrier height ${ }^{[30]}$ that is equal to $\left(\varphi_{s}+\varphi_{t}\right) / 2$, where $\varphi_{s}$ and $\varphi_{t}$ are the work functions of the sample and the tip, respectively. The derivative of this tunneling probability is given by

$$
\frac{d T}{d z}=T(V, E, z)\left(-2 \frac{\sqrt{2 m}}{\hbar} z \sqrt{\varphi_{A}+\frac{e V}{2}-E}\right)=A(V, E) T(V, E, z) .
$$

We emphasize that $A(V, E)$ does not depend on $z$. When we insert Equation (2) of $T(V, E, z)$ in the expression of the current $\mathrm{I}$ in Equation (1), $d I / d z$ is given by

$$
\frac{d I}{d z}=C \int_{0}^{e V} \rho_{t}(E-e V) \rho_{s}(E) \frac{T(V, E, z)}{d z} d E=A(V, E) I .
$$

Here, $d I / d z$ does not depend on $z_{0}$, i.e., the extension of the $z$-piezo, or $z$, the tip-sample distance. For small sample biases, i.e., $\mathrm{eV} / 2 \ll \varphi_{A}$, one finds

$$
\frac{1}{I} \frac{d I}{d z}=\frac{d \ln (I)}{d z} \approx \frac{2 \sqrt{2 m}}{\hbar} I \sqrt{\varphi_{A}} .
$$

The apparent barrier height is related to the local sample work function as stated earlier and is sensitive to the chemical composition of the surface. The measured apparent barrier height has experimentally been found to be independent of the tip-sample distance for reasonable tunnel gaps up until the point of contact. ${ }^{[31]}$ During constant current scanning, the height of the tip is constantly adjusted as to keep a constant tunneling current. To record these $d I / d z$ maps, a small sinusoidal signal (with a frequency that exceeds the bandwidth of the feedback loop) is applied 
to the z-piezo during scanning at constant current. The resulting oscillations in the current can be accurately detected by a lock-in technique.

Simultaneously with the topography in Figure 4.2a, the $d I / d z$ map as shown in Figure 4.2b was obtained. As can be seen, the $d I / d z$ map does not show any contrast besides the atomic corrugation. This atomic corrugation is a result of lateral variations of the decay length of the surface wave functions, as discussed in more detail by Wiesendanger. ${ }^{[30]}$ With the topographic information removed, it is even more clear that there is one continuous lattice. Also, since the topographic information is removed from this scan, we conclude that the electronic properties of the hills and valleys are identical. This would not be the case if the hills were silicon islands because this would result in a contrast variation of the measured apparent barrier height. Therefore, we have to conclude that there is actually no silicon at all on the surface of WSe2. The outer-most layer of the hill and valley structure is pure $\mathrm{WSe}_{2}$, and therefore silicon must have been intercalated in between the top layers of WSe 2 . These results are supported by $I(V)$ spectra recorded on the bare WSe 2 surface before and after the deposition of silicon in Figure 4.3. In order to compare the $I(V)$ traces of the bare $\mathrm{WSe}_{2}$ surface and hills and valleys that are found after deposition, we have taken all $I(V)$ traces with the same set points $(\mathrm{V}=-0.8 \mathrm{~V}$ and $\mathrm{I}=$ $1.5 \mathrm{nA})$. As can be seen in Figure 4.3, all three $I(V)$ curves share the same semiconductor

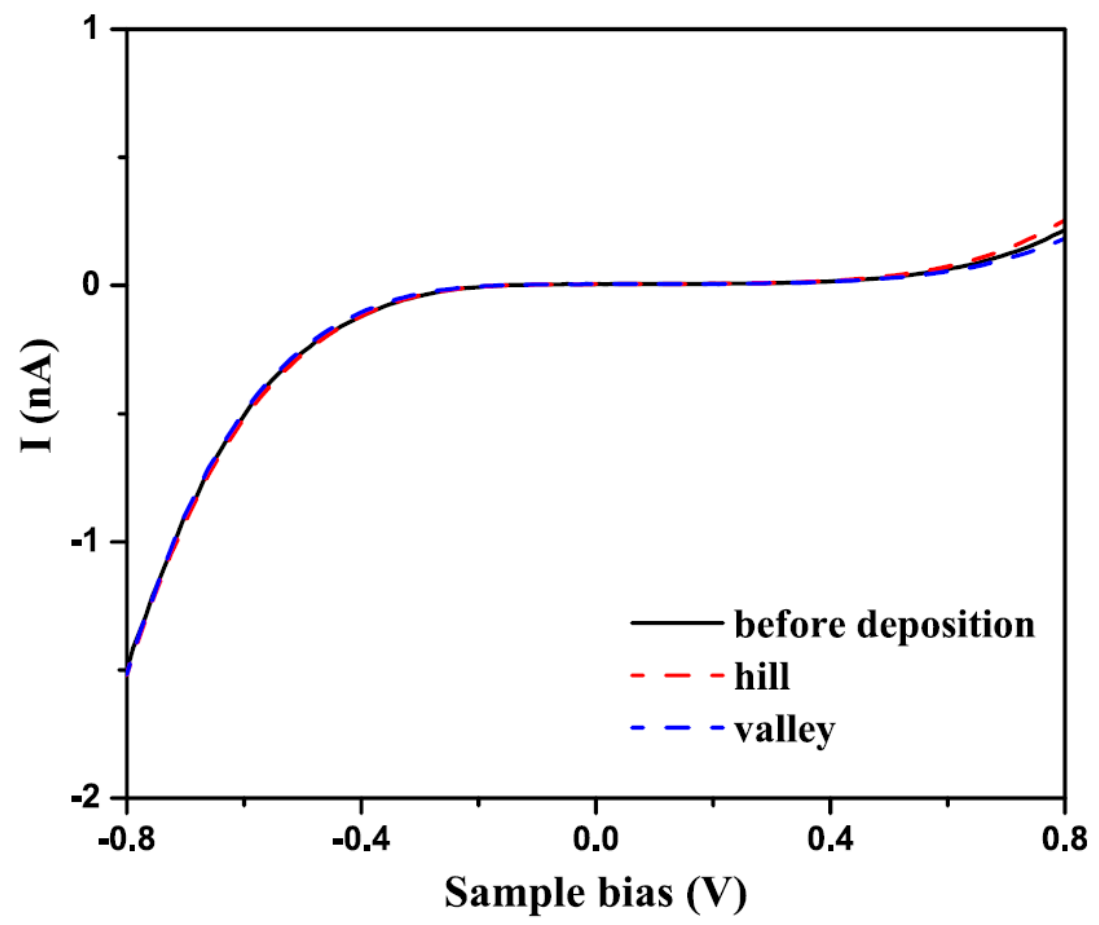

Figure 4.3: $I(V)$ curves recorded on $\mathrm{WSe}_{2}(\mathrm{~V}=-0.8 \mathrm{~V}$ and $\mathrm{I}=1.5 \mathrm{nA})$ before deposition (black curve), after the deposition of 0.25 monolayers of Si on a hill (red curve) and a valley (blue curve). 
characteristics with similar bandgap. Finally, it should be noted that intercalation is ubiquitous in TMDs because intercalated atoms can exchange charge more easily than atoms located on top of the TMD. ${ }^{[32]}$ Intercalation has been found to occur through defects or steps in the surface. $^{[33]}$ The diffusion barrier on van der Waals materials is very low, and therefore the adatoms can easily reach step edges or defects, even though the defect density is rather low. ${ }^{[34]}$ Intercalation in TMDs is the rule rather than the exception for small atoms such as sodium and lithium, ${ }^{[33,35]}$ but even large atoms, such as cesium and gold, can intercalate. ${ }^{[36-38]}$

\subsection{Conclusions}

In summary, the deposition of silicon on $\mathrm{WSe}_{2}$ has been studied with scanning tunneling microscopy and spectroscopy. Upon the deposition of silicon, a hill-and-valley structure develops that has a lattice constant that has the exact same value as the TMD substrate. Spatial maps of the $d I / d z$ provide compelling evidence that silicon does not grow on top of WSe $\mathrm{Cut}_{2}$ rather intercalates between the top layers of $\mathrm{WSe}_{2}$. 


\section{Bibliography}

[1] Novoselov, K. S.; Geim, A. K.; Morozov, S. V.; Jiang, D.; Zhang, Y.; Dubonos, S. V.; Grigorieva, I. V.; Firsov, A. A. Electric Field Effect in Atomically Thin Carbon Films. Science 2004, 306 (5696), 666-669.

[2] Rydberg, H.; Dion, M.; Jacobson, N.; Schröder, E.; Hyldgaard, P.; Simak, S. I.; Langreth, D. C.; Lundqvist, B. I. Van Der Waals Density Functional for Layered Structures. Phys. Rev. Lett. 2003, 91 (12), 126402.

[3] Xu, M.; Liang, T.; Shi, M.; Chen, H. Graphene-Like Two-Dimensional Materials. Chem. Rev. 2013, 113 (5), 3766-3798.

[4] Mak, K. F.; Lee, C.; Hone, J.; Shan, J.; Heinz, T. F. Atomically Thin MoS2: A New DirectGap Semiconductor. Phys. Rev. Lett. 2010, 105 (13), 136805.

[5] Li, H.; Zhang, Q.; Yap, C. C. R.; Tay, B. K.; Edwin, T. H. T.; Olivier, A.; Baillargeat, D. From Bulk to Monolayer $\mathrm{MoS}_{2}$ : Evolution of Raman Scattering. Adv. Funct. Mater. 2012, 22 (7), 1385-1390.

[6] Balog, R.; Jørgensen, B.; Nilsson, L.; Andersen, M.; Rienks, E.; Bianchi, M.; Fanetti, M.; Lægsgaard, E.; Baraldi, A.; Lizzit, S.; et al. Bandgap Opening in Graphene Induced by Patterned Hydrogen Adsorption. Nat. Mater. 2010, 9 (4), 315-319.

[7] Park, J.-S.; Choi, H. J. Band-Gap Opening in Graphene: A Reverse-Engineering Approach. Phys. Rev. B 2015, 92 (4), 045402.

[8] Guzmán-Verri, G. G.; Lew Yan Voon, L. C. Electronic Structure of Silicon-Based Nanostructures. Phys. Rev. B 2007, 76 (7), 075131.

[9] Lalmi, B.; Oughaddou, H.; Enriquez, H.; Kara, A.; Vizzini, S.; Ealet, B.; Aufray, B. Epitaxial Growth of a Silicene Sheet. Appl. Phys. Lett. 2010, 97 (22), 223109. 
[10] Jamgotchian, H.; Colignon, Y.; Hamzaoui, N.; Ealet, B.; Hoarau, J. Y.; Aufray, B.; Bibérian, J. P. Growth of Silicene Layers on Ag(111): Unexpected Effect of the Substrate Temperature. J. Phys. Condens. Matter 2012, 24 (17), 172001.

[11] Vogt, P.; De Padova, P.; Quaresima, C.; Avila, J.; Frantzeskakis, E.; Asensio, M. C.; Resta, A.; Ealet, B.; Le Lay, G. Silicene: Compelling Experimental Evidence for Graphenelike Two-Dimensional Silicon. Phys. Rev. Lett. 2012, 108 (15), 155501.

[12] Enriquez, H.; Vizzini, S.; Kara, A.; Lalmi, B.; Oughaddou, H. Silicene Structures on Silver Surfaces. J. Phys. Condens. Matter 2012, 24 (31), 314211.

[13] Chen, M. X.; Weinert, M. Revealing the Substrate Origin of the Linear Dispersion of Silicene/Ag(111). Nano Lett. 2014, 14 (9), 5189-5193.

[14] Lin, C.-L.; Arafune, R.; Kawahara, K.; Kanno, M.; Tsukahara, N.; Minamitani, E.; Kim, Y.; Kawai, M.; Takagi, N. Substrate-Induced Symmetry Breaking in Silicene. Phys. Rev. Lett. 2013, 110 (7), 076801.

[15] Gao, N.; Li, J. C.; Jiang, Q. Tunable Band Gaps in Silicene--MoS 2 Heterobilayers. Phys. Chem. Chem. Phys. 2014, 16 (23), 11673-11678.

[16] Chiappe, D.; Scalise, E.; Cinquanta, E.; Grazianetti, C.; Broek, B. van den; Fanciulli, M.; Houssa, M.; Molle, A. Two-Dimensional Si Nanosheets with Local Hexagonal Structure on a $\mathrm{MoS}_{2}$ Surface. Adv. Mater. 2014, 26 (13), 2096-2101.

[17] Molle, A.; Lamperti, A.; Rotta, D.; Fanciulli, M.; Cinquanta, E.; Grazianetti, C. Electron Confinement at the Si/MoS 2 Heterosheet Interface. Adv. Mater. Interfaces 2016, 3 (10), 1500619.

[18] Li, L.; Lu, S.; Pan, J.; Qin, Z.; Wang, Y.; Wang, Y.; Cao, G.; Du, S.; Gao, H.-J. Buckled Germanene Formation on Pt(111). Adv. Mater. 2014, 26 (28), 4820-4824. 
[19] Dávila, M. E.; Xian, L.; Cahangirov, S.; Rubio, A.; Lay, G. L. Germanene: A Novel Two-Dimensional Germanium Allotrope Akin to Graphene and Silicene. New J. Phys. 2014, 16 (9), 095002.

[20] Bampoulis, P.; Zhang, L.; Safaei, A.; Gastel, R. van; Poelsema, B.; Zandvliet, H. J. W. Germanene Termination of $\mathrm{Ge}_{2} \mathrm{Pt}$ Crystals on Ge(110). J. Phys. Condens. Matter 2014, 26 (44), 442001.

[21] Acun, A.; Zhang, L.; Bampoulis, P.; Farmanbar, M.; van Houselt, A.; Rudenko, A. N.; Lingenfelder, M.; Brocks, G.; Poelsema, B.; Katsnelson, M. I.; et al. Germanene: The Germanium Analogue of Graphene. J. Phys. Condens. Matter 2015, 27 (44), 443002.

[22] Zhang, L.; Bampoulis, P.; Rudenko, A. N.; Yao, Q.; van Houselt, A.; Poelsema, B.; Katsnelson, M. I.; Zandvliet, H. J. W. Structural and Electronic Properties of Germanene on $\mathrm{MoS}_{2}$. Phys. Rev. Lett. 2016, 116 (25), 256804.

[23] Zhang, L.; Bampoulis, P.; van Houselt, A.; Zandvliet, H. J. W. Two-Dimensional Dirac Signature of Germanene. Appl. Phys. Lett. 2015, 107 (11), 111605.

[24] Schutte, W. J.; De Boer, J. L.; Jellinek, F. Crystal Structures of Tungsten Disulfide and Diselenide. J. Solid State Chem. 1987, 70 (2), 207-209.

[25] Lebègue, S.; Eriksson, O. Electronic Structure of Two-Dimensional Crystals from Ab Initio Theory. Phys. Rev. B 2009, 79 (11), 115409.

[26] Cahangirov, S.; Topsakal, M.; Aktürk, E.; Şahin, H.; Ciraci, S. Two- and OneDimensional Honeycomb Structures of Silicon and Germanium. Phys. Rev. Lett. 2009, 102 (23), 236804.

[27] Roome, N. J.; Carey, J. D. Beyond Graphene: Stable Elemental Monolayers of Silicene and Germanene. ACS Appl. Mater. Interfaces 2014, 6 (10), 7743-7750.

[28] Koma, A.; Sunouchi, K.; Miyajima, T. Fabrication and Characterization of Heterostructures with Subnanometer Thickness. Microelectron. Eng. 1984, 2 (1), 129-136. 
[29] Tersoff, J.; Hamann, D. R. Theory of the Scanning Tunneling Microscope. Phys. Rev. B 1985, 31 (2), 805-813.

[30] Wiesendanger, R.; Roland, W. Scanning Probe Microscopy and Spectroscopy: Methods and Applications; Cambridge University Press, 1994.

[31] Olesen, L.; Brandbyge, M.; Sørensen, M. R.; Jacobsen, K. W.; Lægsgaard, E.; Stensgaard, I.; Besenbacher, F. Apparent Barrier Height in Scanning Tunneling Microscopy Revisited. Phys. Rev. Lett. 1996, 76 (9), 1485-1488.

[32] Whittingham, M. S.; Chianelli, R. R. Layered Compounds and Intercalation Chemistry: An Example of Chemistry and Diffusion in Solids. J. Chem. Educ. 1980, 57 (8), 569.

[33] Xiong, F.; Wang, H.; Liu, X.; Sun, J.; Brongersma, M.; Pop, E.; Cui, Y. Li Intercalation in $\mathrm{MoS}_{2}$ : In Situ Observation of Its Dynamics and Tuning Optical and Electrical Properties. Nano Lett. 2015, 15 (10), 6777-6784.

[34] Petrović, M.; Šrut Rakić, I.; Runte, S.; Busse, C.; Sadowski, J. T.; Lazić, P.; Pletikosić, I.; Pan, Z.-H.; Milun, M.; Pervan, P.; et al. The Mechanism of Caesium Intercalation of Graphene. Nat. Commun. 2013, 4, 2772.

[35] Brauer, H. E.; Starnberg, H. I.; Holleboom, L. J.; Strocov, V. N.; Hughes, H. P. Electronic Structure of Pure and Alkali-Metal-Intercalated VSe2. Phys. Rev. B 1998, 58 (15), 10031-10045.

[36] Pettenkofer, C.; Jaegermann, W.; Schellenberger, A.; Holub-Krappe, E.; Papageorgopoulos, C. A.; Kamaratos, M.; Papageorgopoulos, A. Cs Deposition on Layered

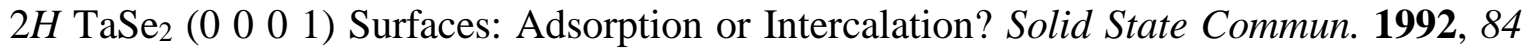
(9), 921-926.

[37] Starnberg, H. I.; Brauer, H. E.; Holleboom, L. J.; Hughes, H. P. 3D-to-2D Transition by Cs Intercalation of VSe 2 . Phys. Rev. Lett. 1993, 70 (20), 3111-3114. 
[38] Iyikanat, F.; Sahin, H.; Senger, R. T.; Peeters, F. M. Ag and Au Atoms Intercalated in Bilayer Heterostructures of Transition Metal Dichalcogenides and Graphene. APL Mater. 2014, 2 (9), 092801. 


\section{Effect of substrate and hydrogenation on germanene}

\subsection{Introduction}

The rise of graphene ${ }^{[1,2]}$ has triggered many scientists to synthesize and study other twodimensional elemental materials. Silicene, germanene, and stanene, i.e., the silicon, germanium, and tin analogues of graphene, are among these two-dimensional materials that have received quite some attention owing to their similarity with graphene. ${ }^{[3-7]}$ Theoretical calculations have revealed that silicene, germanene, and stanene exhibit linear dispersing energy bands in the vicinity of $\mathrm{K}$ and $\mathrm{K}$ ' points of the Brillouin zone. ${ }^{[8-10]}$ These materials are, just as graphene, semimetals that host massless Dirac fermions. In contrast to graphene, these elemental twodimensional materials do not occur in nature, and therefore, they have to be synthesized. Another disadvantage of silicene, germanene, and stanene is that they oxidize, i.e., they are not stable at ambient conditions. Despite these disadvantages, there are also several advantages: (1) the honeycomb lattices of silicene, germanene, and stanene are not planar, as in graphene, but buckled, paving the way for the opening of a bandgap by, for instance, applying an external electric field and (2) the spin-orbit coupling in these materials is much larger than that in graphene, making these two-dimensional materials appealing candidates for spintronic based applications.

\subsubsection{Charge inhomogeneities in 2D material}

An ideal graphene sheet, i.e., a sheet that is undoped, perfectly flat, and completely free from defects and charged impurities, has its charge neutrality point, also referred to as the Dirac point, located at the Fermi level. The density of states of this ideal graphene sheet vanishes at the Dirac point. Recent experiments have revealed that graphene placed on a substrate often exhibits a spatially varying Dirac point, resulting in electron-hole puddles. The exact origin of these charge puddles is still under debate. Possible candidates for the occurrence of these charge puddles are (1) charged impurities in the substrate on which the graphene is placed, ${ }^{[11,12]}(2)$ charged species which are intercalated between the graphene sheet and the substrate, ${ }^{[13]}$ and (3) the local curvature of the graphene sheet. ${ }^{[14-16]}$ The first experimental evidence for the occurrence of charge puddles in two-dimensional materials dates back to 2008. Martin et al. ${ }^{\text {[11] }}$ used a scanning single-electron transistor to spatially map out the charge inhomogeneities in graphene on $\mathrm{SiO}_{2}$. One year later, Zhang et al. ${ }^{[12]}$ used a scanning tunneling microscope to map out the charge density inhomogeneities in graphene on $\mathrm{SiO}_{2}$ by a technique, referred to as Dirac- 
point mapping, with a charge density spatial resolution that is substantially higher than what can be achieved by the scanning single-electron transistor. These authors arrived at the conclusion that the electron-hole puddles originate from charge donating defects and/or impurities in the $\mathrm{SiO}_{2}$ substrate. As demonstrated by Martin et al., . ${ }^{[13]}$ also atoms or molecules intercalated between graphene and its support can result in the formation of charge puddles. Gibertini et al. ${ }^{[15,16]}$ used density functional theory calculations to show that structural corrugations are in principle sufficient to explain the formation of charge puddles in graphene. They also pointed out that the locations of these electron-hole puddles, which have a typical dimension of a few nanometers, do not exhibit a clear correlation with the topography of the graphene sheet. It is worth mentioning that electron-hole puddles are also found in bilayer graphene. ${ }^{[17]}$ So far, charge puddles have only been found in graphene and not in any other elemental two-dimensional material.

In this chapter, we have investigated the electronic disorder of germanene sheets grown on bulk $\mathrm{MoS}_{2}$ by using scanning tunneling microscopy. Inspired by the observation of charge puddles in graphene sheets, we have scrutinized if such charge inhomogeneities are also present in germanene. In order to map out the charge density of the germanene sheet, we have used the aforementioned Dirac point mapping technique.

\subsubsection{From germanene to germanane}

Graphene exhibits many interesting and appealing properties, but unfortunately the material cannot be used for field-effect based electronic devices because it is gapless. In principle, a bandgap can be opened in graphene; however, this usually goes at the expense of the high charge carrier mobilities in graphene. Silicene and germanene are, provided that the rather small spin-orbit gap is ignored, also semimetals. Hydrogenated silicene and germanene, usually referred to as silicane and germanane, respectively, exhibit a sizeable bandgap and still have appreciable charge carrier mobilities. ${ }^{[18-20]}$ In 2013, the first successful synthesis of germanane was reported by Bianco et al. ${ }^{[18]}$ These authors synthesized germanane via the topochemical deintercalation of $\mathrm{CaGe}_{2}$. Germanane sheets can be obtained by exfoliation since germanane belongs to the family of layered van der Waals materials. At ambient conditions, germanane turns out to be very stable and the material only oxidizes in a time span of several months. This stability is an important prerequisite for the usage of germanane in any technological application. The strong potential of germanane for technological applications is fueled by theoretical calculations, which predict a direct bandgap of $1.5 \mathrm{eV}$ and an electron mobility that 
is about five times larger than that of bulk germanium. ${ }^{[19,20]}$ The existence of a bandgap in germanane was confirmed experimentally by Bianco et al. ${ }^{[18]}$ Inspired by the results of Bianco et al., Madhushankar et al. ${ }^{[21]}$ realized a germanane based field-effect transistor. These authors showed that their germanane field effect transistor, which involved a $60 \mathrm{~nm}$ thick stack of germanane layers, exhibited ambipolar transport, but the charge carrier mobilities were much lower $\left(\sim 70 \mathrm{~cm}^{2} / \mathrm{V}\right.$ s at room temperature and $\sim 150 \mathrm{~cm}^{2} / \mathrm{V} \mathrm{s}$ at $\left.77 \mathrm{~K}\right)$ than the theoretical predicted value $\left(20000 \mathrm{~cm}^{2} / \mathrm{V} \mathrm{s}\right)$.

Here, we aim to realize a single germanane layer by hydrogenating germanene synthesized in an ultra-high vacuum environment. The germanene sheets are grown on a substrate, and therefore, only one side of the germanene sheet will be exposed to atomic hydrogen. Since only the Ge atoms of the upward buckled hexagonal sub-lattice will be hydrogenated, the maximum coverage of one monolayer refers to this situation. In this chapter, our scanning tunneling spectroscopy measurements reveal that full hydrogenation results in the opening of a bandgap of about $0.5 \mathrm{eV}$. Further exposure to atomic hydrogen leads to roughening of the germanane layer, which we ascribe to intercalation of the atomic hydrogen.

\subsection{Experimental details}

\subsubsection{Growth of germanene on $\mathrm{MoS}_{2}$}

The scanning tunneling microscopy (STM) and spectroscopy (STS) experiments have been performed at room temperature with an ultra-high vacuum STM (Omicron STM-1). The base pressure of the ultra-high vacuum system is $3 \times 10^{-11}$ mbar. $\mathrm{MoS}_{2}$ samples were freshly cleaved from synthesized $2 \mathrm{H}-\mathrm{MoS}_{2}$ (purchased from 2D Semiconductors) before inserting into the vacuum system. The $\mathrm{MoS}_{2}$ samples are mounted on a Mo sample holder. Germanium was deposited onto the $\mathrm{MoS}_{2}$ substrate, which was held at room temperature, by resistively heating a small piece of a $\mathrm{Ge}(001)$ wafer at a temperature of $\sim 1150 \mathrm{~K}$. Prior to the deposition process, the $\mathrm{Ge}(001)$ wafer was cleaned by outgassing at a temperature of $700 \mathrm{~K}$ for about $24 \mathrm{~h}$ followed by several cycles of argon ion bombardment at $800 \mathrm{eV}$ and annealing at $1100 \mathrm{~K} .{ }^{[22]}$ The Ge source was located at a distance of $\sim 10 \mathrm{~mm}$ from the $\mathrm{MoS}_{2}$ substrate. After the deposition of germanium, the $\mathrm{MoS}_{2}$ sample was inserted into the STM. 


\subsubsection{Hydrogenation of germanene terminated on Ge2Pt nanocrystal}

The experiments are performed in an ultra-high vacuum system that is equipped with a room temperature scanning tunneling microscope (Omicron STM1). The base pressure of the ultrahigh vacuum system is $3 \times 10^{-11} \mathrm{mbar}$. The Ge(110) substrates are nearly intrinsic and nominally flat and have dimensions of $10 \times 4 \times 0.5 \mathrm{~mm}$. The substrates are mounted on a sample holder that only contains molybdenum, tantalum, and aluminum oxide components. After introducing the Ge(110) samples, via a load lock system, into the ultra-high vacuum system, they are carefully degassed at a temperature of $800 \mathrm{~K}$ for at least $24 \mathrm{~h}$. Subsequently, the samples are cleaned by several cycles of argon ion bombardment at $500 \mathrm{eV}$ and annealing at $1100 \mathrm{~K} \cdot{ }^{[22]} \mathrm{Pt}$ is deposited on the $\mathrm{Ge}(110)$ substrate by resistively heating a W wire wrapped with high purity $\mathrm{Pt}(99.995 \%)$. After Pt-deposition, the sample is flash annealed at $1100( \pm 25) \mathrm{K}$ and subsequently cooled down to room temperature before inserting it into the scanning tunneling microscope for imaging. The aforementioned procedure results in a $\mathrm{Ge}(110)$ surface that contains $\mathrm{Ge}_{2} \mathrm{Pt}$ clusters with typical dimensions of a few hundreds of nanometers. ${ }^{[23]}$ There are two types of $\mathrm{Ge}_{2} \mathrm{Pt}$ clusters: pyramids and flat-topped clusters. The flat-topped clusters exhibit a buckled honeycomb lattice with a lattice constant of $4.2 \AA .{ }^{[23]}$ Scanning tunneling spectroscopy measurements show that the germanene sheet possesses a V-shaped density of states, which is one of the hallmarks of a two-dimensional Dirac material. ${ }^{[24]}$ The steps of the germanene sheets on the $\mathrm{Ge}_{2} \mathrm{Pt}$ clusters are quantized in units of $5.6 \pm 0.1 \AA$, i.e., twice the germanene interlayer spacing. ${ }^{[23]}$ The latter suggest that we are dealing with germanene bilayers or multiples thereof, rather than a single germanene layer. This would also explain why the top germanene layer is electronically decoupled from the $\mathrm{Ge}_{2} \mathrm{Pt}$ cluster.

The hydrogen adsorption experiments were performed by exposing the sample at room temperature to high-purity molecular hydrogen at a pressure in the range of $1 \times 10^{-7}-3 \times 10^{-5} \mathrm{mbar}$. The molecular hydrogen was decomposed into atomic hydrogen using a hot tungsten filament, which was heated to a temperature of about $2000 \mathrm{~K}$. The sample was located in the field of view of the hot tungsten filament at a distance of a few centimeters, which is substantially smaller than the mean free path of the atomic hydrogen.

\subsection{Charge puddles in germanene}

In Figure 5.1a and 5.1b, a large-scale scanning tunneling microscopy image and a scanning tunneling spectroscopy spectrum of the pristine $\mathrm{MoS}_{2}$ surface are shown. By examining several 
large-scale scanning tunneling microscopy images, similar to the one shown in Figure. 5.1a, we have found a defect density of about $3 \times 10^{-3} \mathrm{~nm}^{-2}$. This defect density, which includes the dark and the dimmer defects shown in Figure 5.1a, compares well with a previous study of the defects of $\mathrm{MoS}_{2}$ using conductive atom force microscopy by Bampoulis et al. ${ }^{[25]}$ The dark features in Figure 5.1a are defects in the first $\mathrm{MoS}_{2}$ trilayer, whereas the dimmer features are defects located in the second $\mathrm{MoS}_{2}$ trilayer. ${ }^{[25]}$ As shown by Bampoulis et al., ${ }^{[25]}$ both the dark and dim defects are electronic in nature since they only show up in conductive atomic force microscopy images and not in topographic atomic force microscopy images. The differential conductivity spectrum displayed in Figure 5.1b demonstrates that the $\mathrm{MoS}_{2}$ substrate has a sizeable bandgap. The defects in $\mathrm{MoS}_{2}$ have been extensively studied (see, e.g., Ref. 26 and references therein). $\mathrm{MoS}_{2}$ exhibits n-type and p-type defects, which are explained by sulfurdeficient and sulfur-rich regions, respectively. ${ }^{[26]}$
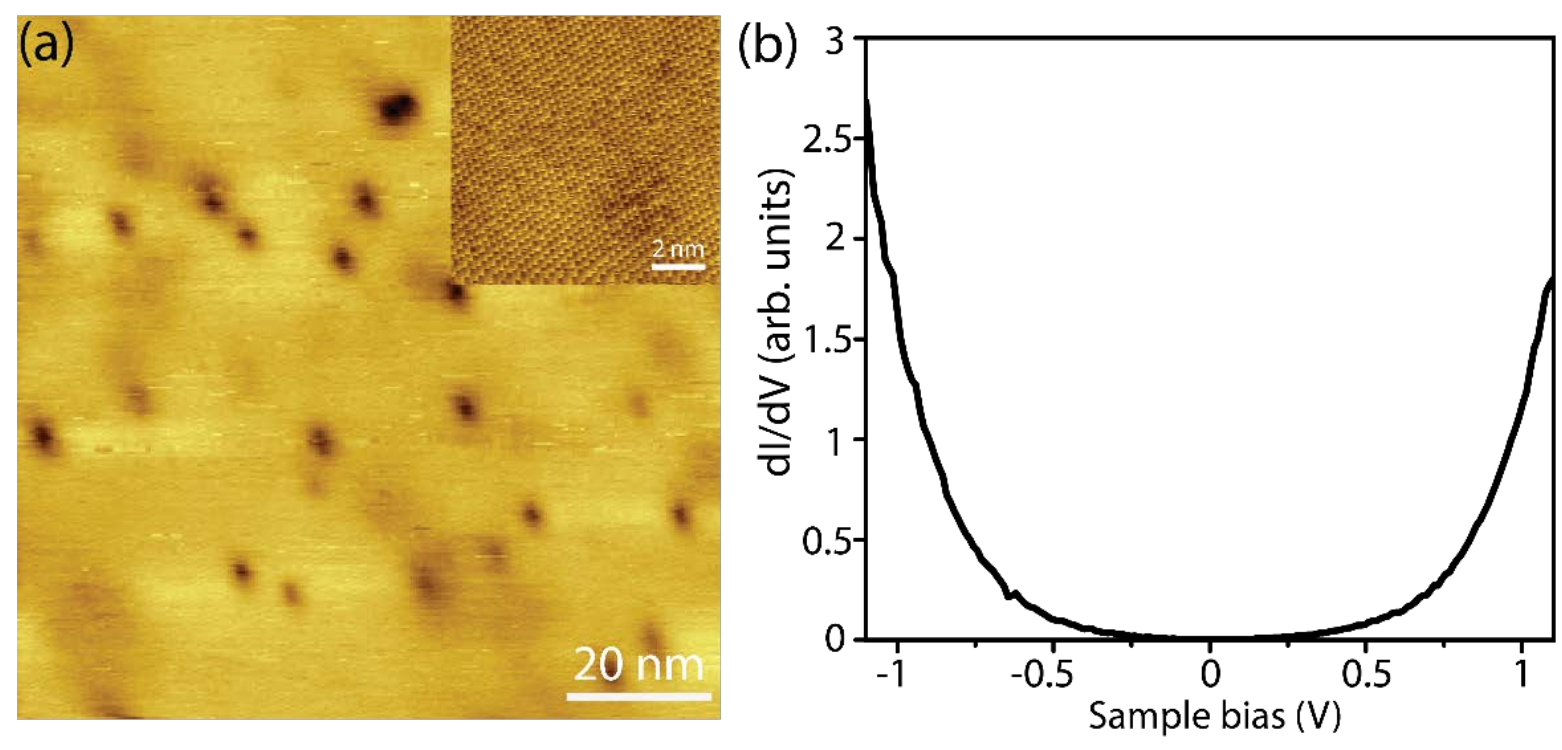

Figure 5.1: (a) Scanning tunneling microscopy image of the pristine $\mathrm{MoS}_{2}$ surface. Inset: small scale scanning tunneling microscopy image. The sample bias is $-0.3 \mathrm{~V}$, and the tunnel current is $500 \mathrm{pA}$. (b) $d I / d V$ spectrum of the pristine $\mathrm{MoS}_{2}$ surface. The set point values are $-1.1 \mathrm{~V}$ and 500 pA.

In Figure 5.2a, a scanning tunneling microscopy image of a germanene layer grown on $\mathrm{MoS}_{2}$ is shown. As we have already shown in a recent study, ${ }^{[5]} \mathrm{Ge}$ atoms deposited on the $\mathrm{MoS}_{2}$ substrate nucleate at pre-existing defects of the $\mathrm{MoS}_{2}$ surface. The germanene islands have a height of $3.2 \AA$ and exhibit a hexagonal symmetry with a lattice constant of 3.8-3.9 $\AA$, which 
is about 20\% larger than the lattice constant of pristine $\mathrm{MoS}_{2}$. This relatively large interlayer spacing of $3.2 \AA$ between the germanene sheet and the $\mathrm{MoS}_{2}$ substrate hints to a relatively weak interaction between the layers as one expects for materials that are held together by Van der Waals interactions. The deposition of more Ge eventually leads to a complete and very flat germanene layer [see Figure 5.2a]. ${ }^{[5]}$ Owing to the large buckling of germanene on $\mathrm{MoS}_{2}$, only one of the two triangular sub-lattices is visible in the scanning tunneling microscopy images [see the inset of Figure 5.2a]. The electronic properties of the germanene layer have been studied by recording grid $I(V)$ curves with the feedback loop disabled. The typical grid size is $60 \times 60$ for a $100 \mathrm{~nm}$ by $100 \mathrm{~nm}$ scan. The differential conductivity ( $d I / d V)$, which is proportional
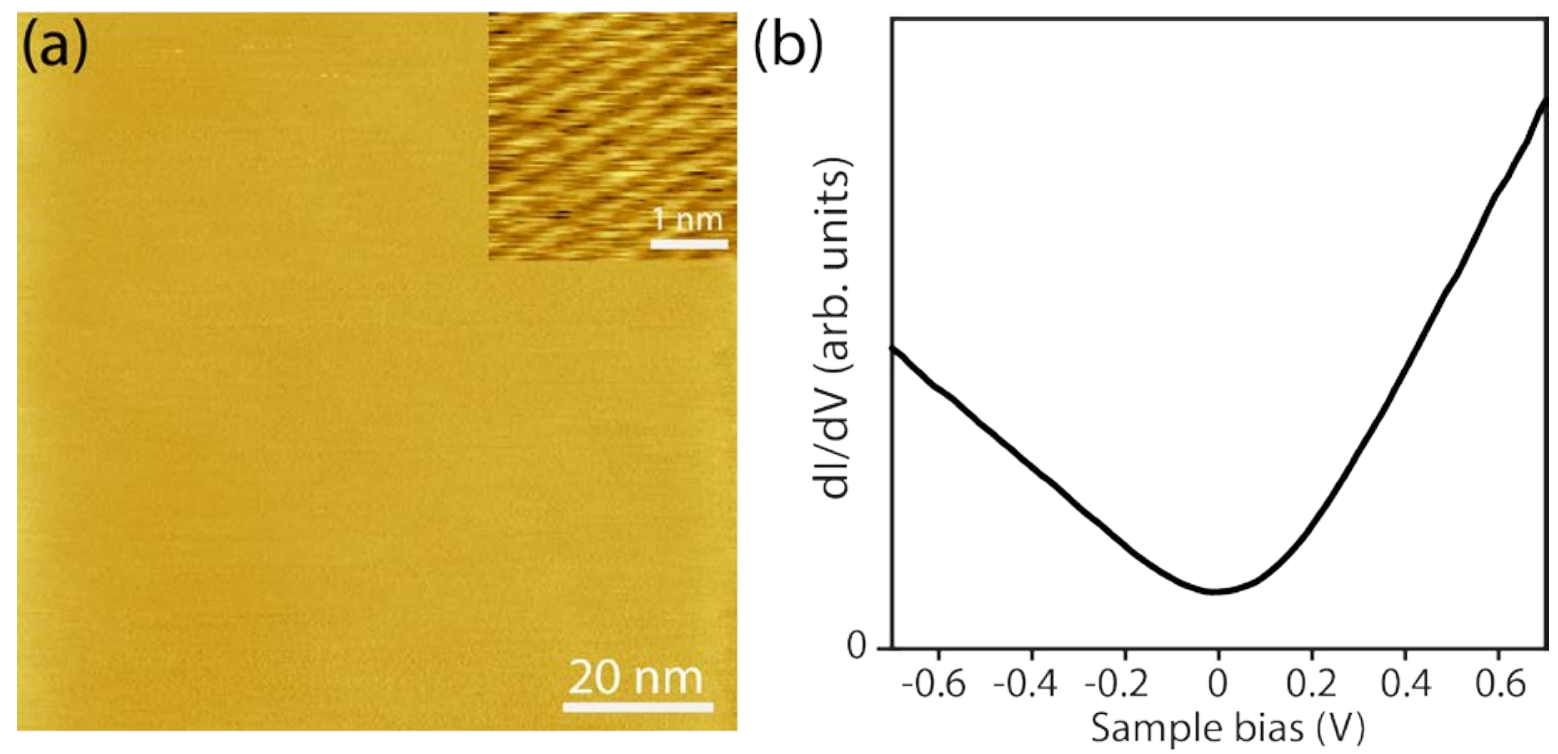

Figure 5.2: (a) Large scale scanning tunneling microscopy image (100 nm by $100 \mathrm{~nm}$ ) of a complete germanene layer grown on $\mathrm{MoS}_{2}$. Inset: small scale scanning tunneling microscopy image of the germanene layer. The sample bias is $-1 \mathrm{~V}$, and the tunnel current is $300 \mathrm{pA}$. (b) Differential conductivity of germanene. The set point values are $-1.4 \mathrm{~V}$ and $600 \mathrm{pA}$.

to the density of states, is obtained by numerically differentiating the $I(V)$ curves. The averaged $d I / d V$ curve is shown in Figure 5.2b. The differential conductivity displays a V-shape, which is one of the signatures of a two-dimensional Dirac material. The density of states does not completely vanish as the Dirac point. The $d I / d V$ curve is asymmetric, a feature that we have observed before. ${ }^{[5]}$ The asymmetry of the $\mathrm{dI} / \mathrm{dV}$ curve could be caused by the electronic structure of the STM tip. In addition, it should be noted that the rounded shape near the Dirac point cannot be fully explained by thermal broadening. A much improved fit is, however, 
obtained by introducing a bandgap of about $25 \mathrm{meV}$. This bandgap could be due to the presence of a spin-orbit gap in germanene. ${ }^{[27]}$ Density functional theory calculations shown in Ref. 5 reveal that besides the linear bands at the $\mathrm{K}$ and $\mathrm{K}$ ' points of the Brillouin zone, there are also two parabolic bands in the vicinity of the Fermi level at the $\Gamma$ point. It is very well possible that these two parabolic bands near the $\Gamma$ point are responsible for the non-zero density of states at the Dirac point; however, the non-zero density of states could also be caused by the electronic structure of the scanning tunneling microscopy tip.

Since the $d I / d V$ curve shown in Figure 5.2b is averaged over 3600 different positions on the surface, it is worthwhile to have a detailed look at the spatial variation of the $d I / d V$ curves. In order to do this, we have determined the exact position of the minimum of all individual $d I / d V$ curves. The minimum of the $\mathrm{V}$-shaped $d I / d V$ curve is located at the charge neutrality point, i.e., the Dirac point. As we will show below, the Dirac point provides information on the local charge density. The dispersion relation of a two-dimensional Dirac material is $E=\hbar v_{F}|k|$, where $\hbar$ is the reduced Planck constant, $v_{F}$ the Fermi velocity, $E$ the energy of the electron, and $k$ the momentum of the electron. The density of states of a two-dimensional Dirac material is given by

$$
D(E)=\frac{2\left|E-E_{D}\right|}{\pi \hbar^{2} v_{F}^{2}}
$$

where $E_{D}$ is the Dirac point energy. The map of the Dirac point energy can be converted into a map of the charge density by using

$$
\rho(x, y)=\int_{0}^{E_{D}} \frac{2\left|E-E_{D}\right|}{\pi \hbar^{2} v_{F}^{2}} d E=-\frac{E_{D}{ }^{2}}{\pi \hbar^{2} v_{F}^{2}} \operatorname{sign}\left(E_{D}\right) .
$$

In Figure 5.3a and 5.3b, spatial maps of the Dirac and charge character of a $100 \mathrm{~nm}$ by $100 \mathrm{~nm}$ germanene/MoS 2 are shown, respectively. The images consist of $60 \times 60$ pixels, and each pixel corresponds to a single $I(V)$ curve. It should be emphasized here that we are dealing with raw data, and we have not applied any smoothening of the $I(V)$ curves in order to determine the minimum of each $I(V)$ curve. The spatial maps of the variation of the Dirac point and charge character reveal that the germanene sheet consists of electron and hole puddles with a typical radius of 10-20 nm embedded in an intrinsic, i.e., undoped, background. The electrostatic screening in two-dimensional Dirac materials is significantly different from electrostatic screening in conventional two-dimensional electron systems. The Dirac point varies from -30 

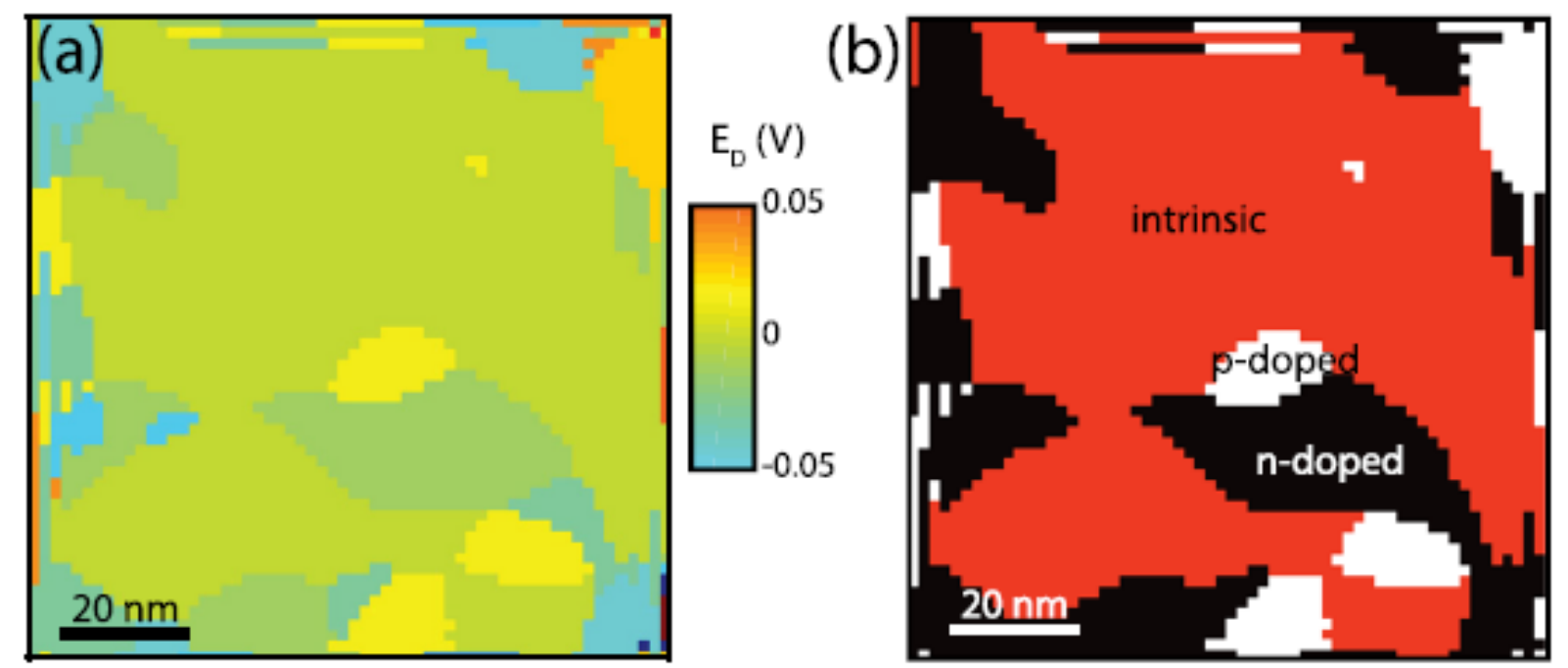

Figure 5.3: (a) Spatial map (60×60 pixels) of the Dirac point of germanene/MoS 2 . The image size is $100 \mathrm{~nm}$ by $100 \mathrm{~nm}$. The color code refers to the position of the Dirac point. (b) Spatial map (60×60 pixels) of the charge character of germanene/MoS 2 (red: intrinsic, black: n-type, and white: p-type). The image size is $100 \mathrm{~nm}$ by $100 \mathrm{~nm}$.

$\mathrm{meV}$ to $15 \mathrm{meV}$ corresponding to a charge fluctuation of $2.6 \times 10^{-3}$ electrons $/ \mathrm{nm}^{2}$ to $6.6 \times 10^{-4}$ holes $/ \mathrm{nm}^{2}$, assuming a Fermi velocity of $5 \times 10^{5} \mathrm{~m} / \mathrm{s} .{ }^{[28]}$ Since the germanene sheet is atomically flat on a length scale exceeding the typical size of the charge puddles, we have to rule out the possibility that charge puddles are caused by structural corrugations or bending of the germanene sheet. The germanene sheet is grown and subsequently studied at ultra-high vacuum conditions, and therefore, we can also rule out the intercalation of atoms or molecules as being the cause of the charge puddles. The only remaining sources are charge donating impurities in the $\mathrm{MoS}_{2}$ substrate. By analyzing several large-scale scanning tunneling microscopy images of our pristine and freshly cleaved $\mathrm{MoS}_{2}$ substrate, we find a defect density of $3 \times 10^{-3} \mathrm{~nm}^{-2}$. This value compares favorably well with the charge puddle density we have determined for the germanene sheet. Based on these findings, we suggest that the charge puddles are caused by charge donating defects of the $\mathrm{MoS}_{2}$ substrate. The typical radius of the charge puddles in germanene is about 10-20 nm. A crude estimate for the size of the charge puddle is the ThomasFermi screened length. In conventional two-dimensional electron systems, the density of states in energy space is constant, and therefore, the Thomas-Fermi screening length, which is inversely proportional to the density of states at the Fermi level, is constant. ${ }^{[29-32]}$ In twodimensional Dirac systems, however, the density of states is proportional to the energy. This results in a Thomas-Fermi screening length of $2 \pi \kappa \hbar v_{F} / 4 e^{2} \sqrt{\pi n}$, where $\mathrm{n}$ is the charge 
density. ${ }^{[29]}$ Assuming a Fermi velocity of $5 \times 10^{5} \mathrm{~m} / \mathrm{s}$ (Refs. 26 and 33) and a charge density of $\sim 3 \times 10^{-3} \mathrm{~nm}^{-2}$ (one charge carrier per defect), we find for germanene on molybdenum disulfide a Thomas-Fermi length of $\sim 10 \mathrm{~nm}$, which is somewhat smaller than our experimental observations.

In addition, the size of the charge puddles in germanene is very comparable to the size of the charge puddles found in graphene at a comparable charge density (see Ref. 12). Please note that the charge density in Ref. 13 is substantially larger than in our case and Ref. 12, resulting in, as expected, smaller charge puddles. In a previous study, ${ }^{[5]}$ we found a qualitative good agreement between the $d I / d V$ spectrum and the density functional theory calculations. There is, however, an overall energy shift of about $0.3 \mathrm{eV}$ between the experimental and theoretical spectra. In Ref. 5 , we tentatively ascribed this energy shift to the presence of defects and/or impurities with an acceptor character. In this work, we have not found any evidence for the presence of these acceptor types of defects and/or impurities in the germanene layer, and therefore, we have to discard this interpretation.

\subsection{Bandgap opening in hydrogenated germanene}

In Figure 5.4a, a scanning tunneling microscopy image of a flat-topped $\mathrm{Ge}_{2} \mathrm{Pt}$ cluster coated with a germanene layer is shown. In the inset, a small scale image of the flat-topped part of the $\mathrm{Ge}_{2} \mathrm{Pt}$ cluster is shown. The lattice constant of the hexagonal structure is $4.2 \pm 0.1 \AA$, which agrees well with the findings of Bampoulis et al. ${ }^{[23]}$ [see Figure 5.4b]. Unfortunately, the resolution of this image is insufficient to resolve the downward buckled Ge atoms. The differential conductivity is shown in Figure 5.4c. The observed V-shaped differential conductivity is in good agreement with the results obtained by Zhang et al. ${ }^{[24]}$

Upon the exposure of the germanene sheet to $100 \mathrm{~L}$ of atomic hydrogen at room temperature, the $(1 \times 1)$ hexagonal structure of germanene changes into a $(2 \times 2)$ hexagonal structure [see Figure. $5.5 \mathrm{a}]$. The $(2 \times 2)$ hexagonal structure still exhibits some defects and adsorbates. The line scan displayed in Figure. 5.5b shows a lattice constant of $0.84 \mathrm{~nm}$, i.e., two times the lattice constant of germanene. The differential conductivity is shown in Figure 5.5c. The hydrogenation results in the opening of a small bandgap of about $0.2 \mathrm{eV}$. Based on the observed $(2 \times 2)$ structure and the small bandgap opening, we suggest that only half of the upward buckled Ge atoms are hydrogenated, resulting in a hydrogen coverage of 1/2 monolayer (at 1 monolayer coverage, all the Ge atoms of the upward buckled hexagonal sub-lattice of the germanene sheet 
are hydrogenated). Upon further exposure to atomic hydrogen, the $(2 \times 2)$ structure vanishes and the bandgap opens further.

(a)

(b)
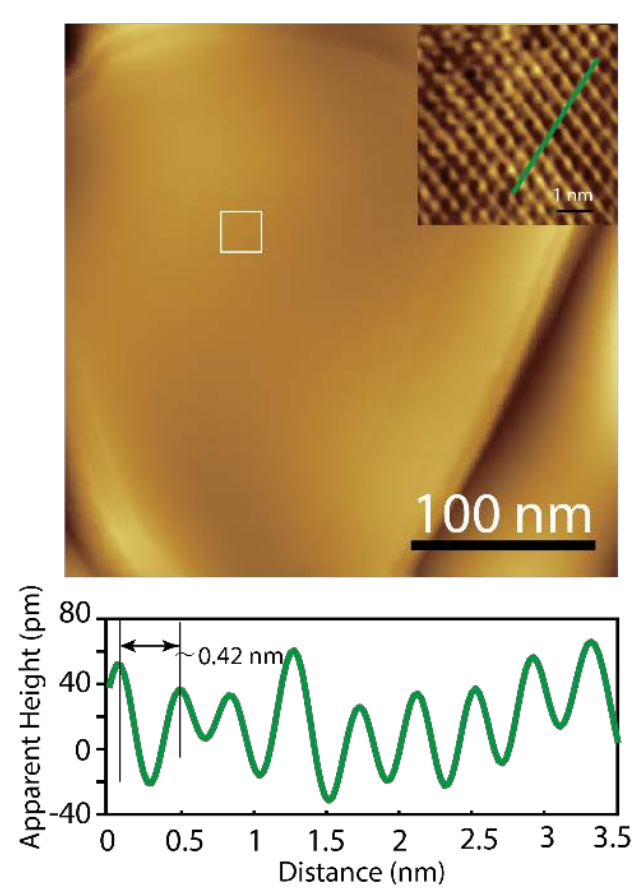

(c)

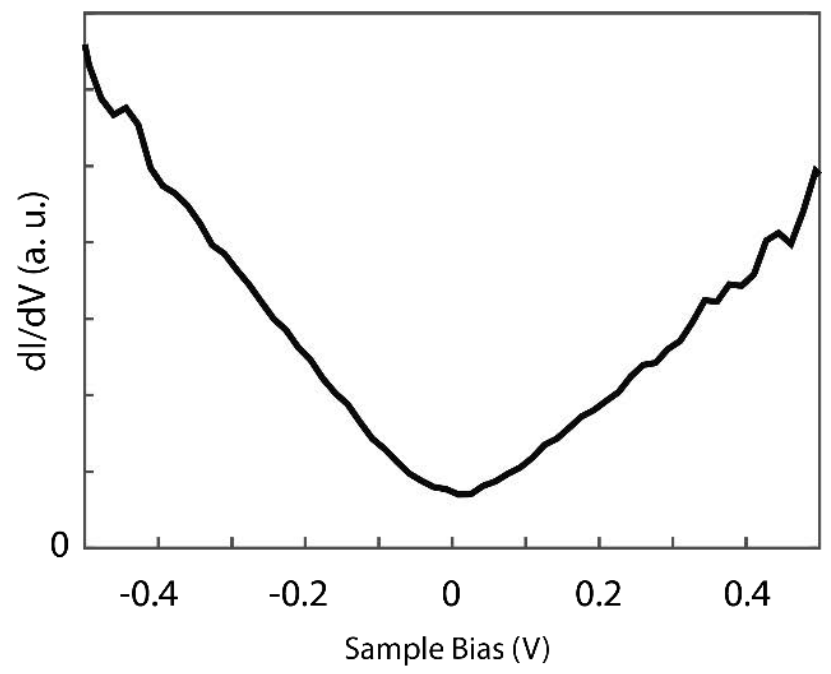

Figure 5.4: (a) Scanning tunneling microscopy image of a germanene coated flat-topped $\mathrm{Ge}_{2} \mathrm{Pt}$ cluster. Sample bias: $-1.5 \mathrm{~V}$ and tunneling current: $0.6 \mathrm{nA}$. Inset: small scale image of the flattopped part of the germanene coated flat-topped $\mathrm{Ge}_{2} \mathrm{Pt}$ cluster. Sample bias: $0.4 \mathrm{~V}$ and tunneling current: $0.4 \mathrm{nA}$. (b) Line profile of the line shown in the inset of graph (a). (c) Differential conductivity $(d I / d V)$ of the germanene coated flat-topped $\mathrm{Ge}_{2}$ Pt cluster. Set points: sample bias $-1.5 \mathrm{~V}$ and tunneling current $1.0 \mathrm{nA}$. 
(a)

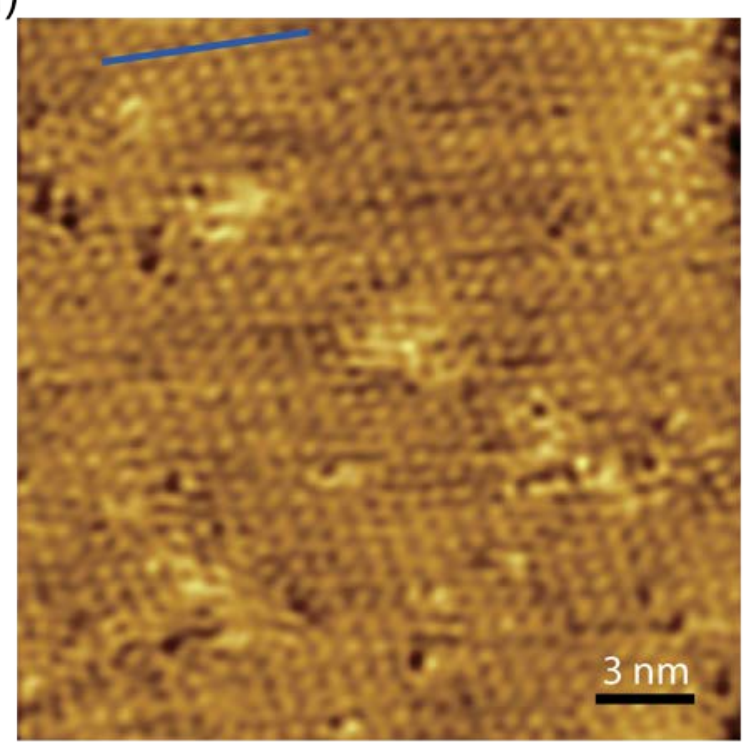

(b)

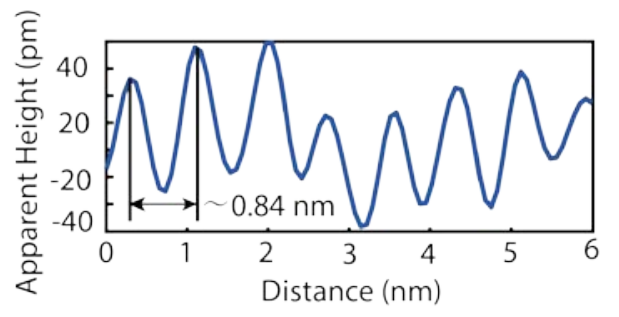

(c)

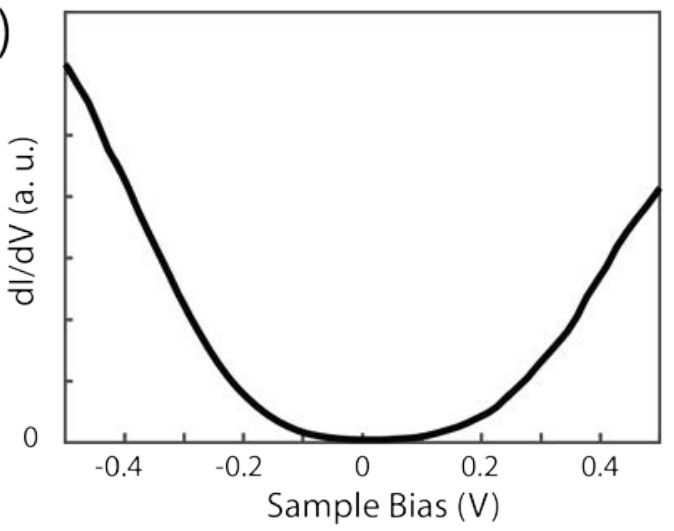

Figure 5.5: (a) Scanning tunneling microscopy image of a partly hydrogenated germanene sheet. The lattice constant of the hexagonal structure is $0.84 \mathrm{~nm}$, i.e., twice the value of the pristine germanene sheet. Sample bias: $-1.5 \mathrm{~V}$ and tunneling current: $1.0 \mathrm{nA}$. Hydrogen exposure of 100 L. (b) Line profile of the line shown in graph (a). (c) Differential conductivity of the partly hydrogenated germanene. Set points: sample bias $-1.0 \mathrm{~V}$ and tunneling current 0.9 nA.

In Figure 5.6b the differential conductivity of the hydrogenated germanene after an exposure of $9000 \mathrm{~L}$ is shown. The bandgap opening amounts about $0.5 \mathrm{eV}$, and the hydrogenated germanene has become slightly n-type. The surface structure exhibits an increase in roughness and disorder, which we ascribe to the intercalation of hydrogen atoms. An scanning tunneling microscopy image is shown in Figure 5.6a. The height variation in this image is about $1 \mathrm{~nm}$. Despite numerous attempts, we were unable to achieve atomically resolved scanning tunneling microscopy images, which is probably caused by the increase in roughness and disorder. The exposure to more atomic hydrogen leads to a further increase in the roughness and size of the bandgap. As a final remark, we would like to emphasize that we have not observed any significant spatial variation of the bandgap, indicating that the bandgap cannot be explained by a spatially varying Dirac point. ${ }^{[3]}$ The hydrogenation of germanene and silicene has been studied quite extensively, see Refs. 20 and 34-36 and Refs. 37 and 38 for several theoretical and experimental articles, respectively. Houssa et al. ${ }^{[20]}$ calculated the electronic band structure 
(a)

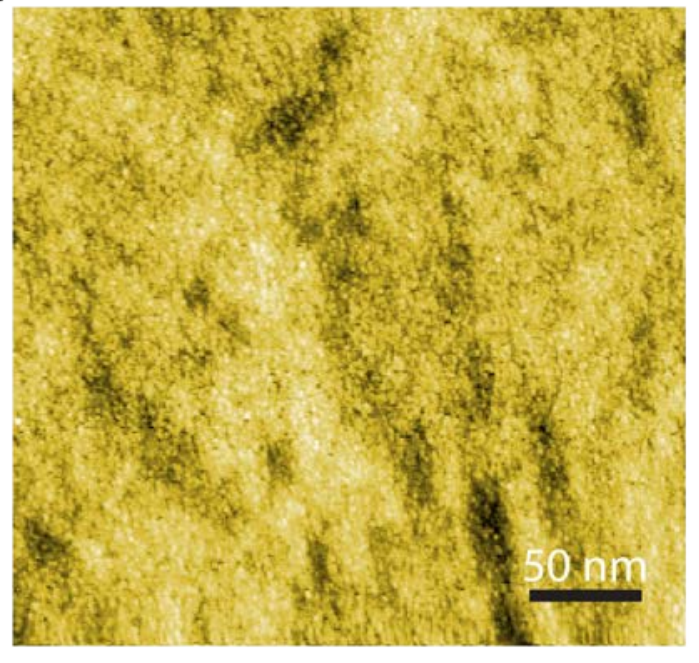

(b)

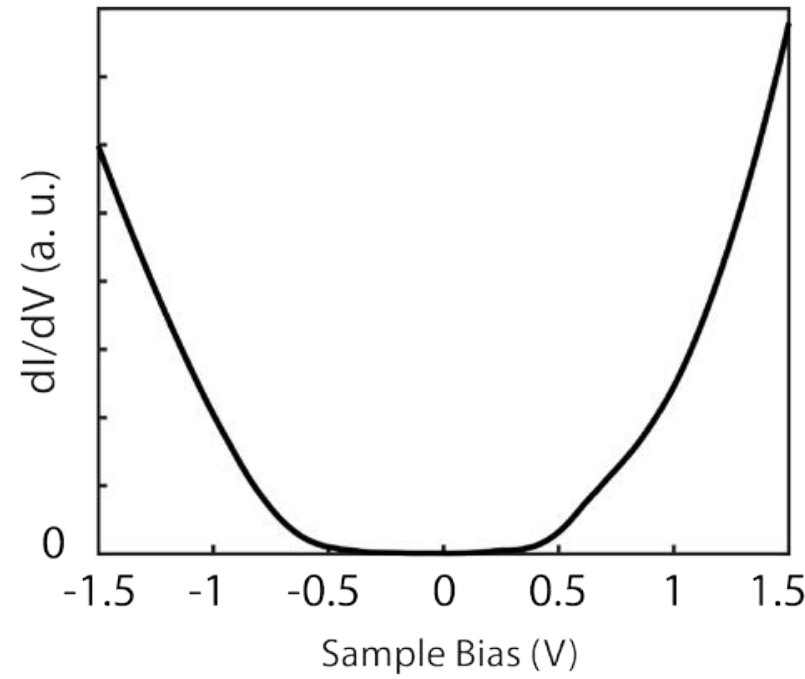

Figure 5.6: (a) Scanning tunneling microscopy image of the fully hydrogenated germanene sheet. Image size $300 \mathrm{~nm} \times 300 \mathrm{~nm}$, sample bias $-0.8 \mathrm{~V}$, and tunnel current $0.9 \mathrm{nA}$. (b) Differential conductivity of a fully hydrogenated germanene (1 monolayer of hydrogen). Set points: sample bias $-1.5 \mathrm{~V}$ and tunneling current $0.9 \mathrm{nA}$. The hydrogen exposure is $9000 \mathrm{~L}$.

of germanane using first-principles total energy calculations based on the density functional theory. They found that the bandgap in germanane is direct, independent of the exact atomic configuration, making this material an appealing candidate for optoelectronic applications. Wang et al. ${ }^{[35]}$ theoretically studied the properties of half-hydrogenated germanene. These authors found that half-hydrogenated germanene is stable and has a direct bandgap. They also pointed out that the buckling as well as the lattice constant of germanene increases upon hydrogenation. Nijamudheen et al. ${ }^{[36]}$ demonstrated that the buckling of germanene results in an enhanced chemical reactivity of germanene for hydrogen. To date, there are, unfortunately, no experimental reports on the hydrogenation of a single sheet of germanene. Luckily, a few experimental studies on the hydrogenation of silicene have been performed recently. The first experimental report on the hydrogenation is by Qiu et al. ${ }^{[37]}$ These authors studied the hydrogenation of a silicene layer synthesized on a $\mathrm{Ag}(111)$ substrate using scanning tunneling microscopy and density functional theory calculations. They focused on the $(3 \times 3)$ silicene structure [a (3×3) silicene supercell is commensurable with a $(4 \times 4)$ cell of the $\mathrm{Ag}(111)$ surface]. Six out of the $18 \mathrm{Si}$ atoms of a (3×3) silicene unit cell are located on-top, or almost on-top, of a Ag atom and are therefore found in the upward buckled position. The other 12 atoms are found in a downward buckled position. Upon hydrogenation, the regular $(3 \times 3)$ cell, also 
referred to as the $\alpha-(3 \times 3)$ structure, converts to a $\gamma-(3 \times 3)$ structure. This $\gamma-(3 \times 3)$ structure is composed of two distinctly different half unit cells, where one half unit cell has 6 and the other half unit cell has only one upward buckled Si atom. ${ }^{[37}$ The hydrogen atoms only adsorb on these upward buckled Si atoms, resulting in a saturation coverage of 7/18 monolayer. The hydrogen adsorption favors the $\mathrm{sp}^{3}$ hybridization, leading to an enhancement of the upward buckling and lattice constant. In a second study, Qiu et al. ${ }^{[38]}$ studied the hydrogenation of the $(2 \sqrt{3} \times 2 \sqrt{3}) \mathrm{R} 30^{\circ}$ silicene phase, which is also commonly found on the $\mathrm{Ag}(111)$ surface. In this case, the hydrogen atoms only adsorb on one of the two sublattices of silicene, yielding a perfect half-hydrogenated $(1 \times 1)$ structure. Based on our experimental results and the available theoretical and experimental data, we arrive at the description of the hydrogenation process of germanene coated $\mathrm{Ge}_{2} \mathrm{Pt}$ crystals. At small hydrogen exposures, the hydrogen atoms only occupy the upward buckled Ge atoms. The adsorption of the hydrogen atom on an upward buckled Ge atom leads to an increase in the buckling as well as a small expansion of the surface lattice constant of germanene. The adsorption of hydrogen leads to the development of a compressive surface stress in the germanene sheet. In order to relieve this surface stress, it is energetically favorable to maximize the next-nearest distance of the hydrogenated Ge atoms. At a coverage of $1 / 2$ monolayer, this results in a $(2 \times 2)$ structure. The next phase of the hydrogenation (1/2 to 1 monolayer) process proceeds substantially slower since now also the energetically unfavorable upward buckled Ge atoms need to be hydrogenated. This results in a further increase in the compressive surface stress. Eventually, the germanene sheet becomes fully hydrogenated and exhibits a sizeable bandgap of $0.5 \mathrm{eV}$. Upon further exposure to hydrogen, we anticipate that hydrogen atoms start to intercalate underneath the halfhydrogenated germanene sheet, leading to an increase in the roughness and a further opening of the bandgap.

\subsection{Conclusions}

we have studied the charge inhomogeneities of germanene grown on $\mathrm{MoS}_{2}$. The charge fluctuations in germanene have been mapped out by using a Dirac point map. Spatial maps of the Dirac point and the charge character reveal that the germanene sheet consists of electrons and hole puddles with a typical radius of 10-20 nm embedded in an intrinsic, i.e., undoped, background. The typical charge per puddle is of the order of one electron or hole. The defect density of $\mathrm{MoS}_{2}$ is determined by the analysis of several large-scale scanning tunneling microscopy images and compares very well with the charge density of the germanene sheet. 
We found strong indications that the charge puddles in germanene are caused by chargedonating defects and impurities in the $\mathrm{MoS}_{2}$ substrate. Our results demonstrate that even in van der Waals heteroepitaxy, the quality of the substrate plays a key role. Charge donating defects or impurities result in electronic inhomogeneities in the two-dimensional material. In addition, hydrogenation of germanene, synthesized on $\mathrm{Ge}_{2} \mathrm{Pt}$, results in the opening of a bandgap of about $0.5 \mathrm{eV}$. At a fractional hydrogen coverage, the $(1 \times 1)$ germanene structure converts to a $(2 \times 2)$ structure with a bandgap opening of about $0.2 \mathrm{eV}$. A further increase in the hydrogen exposure leads to vanishing of the $(2 \times 2)$ structure and an increase in the bandgap opening to about 0.5 $\mathrm{eV}$. Eventually, the roughness and disorder of the germanane sheet increase, which we ascribe to the intercalation of atomic hydrogen. 


\section{Bibliography}

[1] Novoselov, K. S.; Geim, A. K.; Morozov, S. V.; Jiang, D.; Zhang, Y.; Dubonos, S. V.; Grigorieva, I. V.; Firsov, A. A. Electric Field Effect in Atomically Thin Carbon Films. Science 2004, 306 (5696), 666-669.

[2] Geim, A. K.; Novoselov, K. S. The Rise of Graphene. Nat. Mater. 2007, 6 (3), 183-191.

[3] Vogt, P.; De Padova, P.; Quaresima, C.; Avila, J.; Frantzeskakis, E.; Asensio, M. C.; Resta, A.; Ealet, B.; Le Lay, G. Silicene: Compelling Experimental Evidence for Graphenelike Two-Dimensional Silicon. Phys. Rev. Lett. 2012, 108 (15), 155501.

[4] Fleurence, A.; Friedlein, R.; Ozaki, T.; Kawai, H.; Wang, Y.; Yamada-Takamura, Y. Experimental Evidence for Epitaxial Silicene on Diboride Thin Films. Phys. Rev. Lett. 2012, 108 (24), 245501.

[5] Zhang, L.; Bampoulis, P.; Rudenko, A. N.; Yao, Q.; van Houselt, A.; Poelsema, B.; Katsnelson, M. I.; Zandvliet, H. J. W. Structural and Electronic Properties of Germanene on $\mathrm{MoS}_{2}$. Phys. Rev. Lett. 2016, 116 (25), 256804.

[6] Acun, A.; Zhang, L.; Bampoulis, P.; Farmanbar, M.; van Houselt, A.; Rudenko, A. N.; Lingenfelder, M.; Brocks, G.; Poelsema, B.; Katsnelson, M. I.; et al. Germanene: The Germanium Analogue of Graphene. J. Phys. Condens. Matter 2015, 27 (44), 443002.

[7] Zhu, F.; Chen, W.; Xu, Y.; Gao, C.; Guan, D.; Liu, C.; Qian, D.; Zhang, S.-C.; Jia, J. Epitaxial Growth of Two-Dimensional Stanene. Nat. Mater. 2015, 14 (10), 1020-1025.

[8] Takeda, K.; Shiraishi, K. Theoretical Possibility of Stage Corrugation in Si and Ge Analogs of Graphite. Phys. Rev. B 1994, 50 (20), 14916-14922.

[9] Guzmán-Verri, G. G.; Lew Yan Voon, L. C. Electronic Structure of Silicon-Based Nanostructures. Phys. Rev. B 2007, 76 (7), 075131. 
[10] Cahangirov, S.; Topsakal, M.; Aktürk, E.; Şahin, H.; Ciraci, S. Two- and OneDimensional Honeycomb Structures of Silicon and Germanium. Phys. Rev. Lett. 2009, 102 (23), 236804.

[11] Martin, J.; Akerman, N.; Ulbricht, G.; Lohmann, T.; Smet, J. H.; von Klitzing, K.; Yacoby, A. Observation of Electron-Hole Puddles in Graphene Using a Scanning SingleElectron Transistor. Nat. Phys. 2008, 4 (2), 144-148.

[12] Zhang, Y.; Brar, V. W.; Girit, C.; Zettl, A.; Crommie, M. F. Origin of Spatial Charge Inhomogeneity in Graphene. Nat. Phys. 2009, 5 (10), 722-726.

[13] Martin, S. C.; Samaddar, S.; Sacépé, B.; Kimouche, A.; Coraux, J.; Fuchs, F.; Grévin, B.; Courtois, H.; Winkelmann, C. B. Disorder and Screening in Decoupled Graphene on a Metallic Substrate. Phys. Rev. B 2015, 91 (4), 041406.

[14] Deshpande, A.; Bao, W.; Miao, F.; Lau, C. N.; LeRoy, B. J. Spatially Resolved Spectroscopy of Monolayer Graphene on $\$\{\backslash \operatorname{text}\{\mathrm{SiO}\}\}_{-}\{2\} \$$. Phys. Rev. B 2009, 79 (20), 205411.

[15] Gibertini, M.; Tomadin, A.; Polini, M.; Fasolino, A.; Katsnelson, M. I. Electron Density Distribution and Screening in Rippled Graphene Sheets. Phys. Rev. B 2010, 81 (12), 125437.

[16] Gibertini, M.; Tomadin, A.; Guinea, F.; Katsnelson, M. I.; Polini, M. Electron-Hole Puddles in the Absence of Charged Impurities. Phys. Rev. B 2012, 85 (20), 201405.

[17] Rutter, G. M.; Jung, S.; Klimov, N. N.; Newell, D. B.; Zhitenev, N. B.; Stroscio, J. A. Microscopic Polarization in Bilayer Graphene. Nat. Phys. 2011, 7 (8), 649-655.

[18] Bianco, E.; Butler, S.; Jiang, S.; Restrepo, O. D.; Windl, W.; Goldberger, J. E. Stability and Exfoliation of Germanane: A Germanium Graphane Analogue. ACS Nano 2013, 7 (5), $4414-4421$

[19] Lew Yan Voon, L. C.; Sandberg, E.; Aga, R. S.; Farajian, A. A. Hydrogen Compounds of Group-IV Nanosheets. Appl. Phys. Lett. 2010, 97 (16), 163114. 
[20] Houssa, M.; Scalise, E.; Sankaran, K.; Pourtois, G.; Afanas’ev, V. V.; Stesmans, A. Electronic Properties of Hydrogenated Silicene and Germanene. Appl. Phys. Lett. 2011, 98 (22), 223107.

[21] Madhushankar, B. N.; Kaverzin, A.; Giousis, T.; Potsi, G.; Gournis, D.; Rudolf, P.; Blake, G. R.; van der Wal, C. H.; van Wees, B. J. Electronic Properties of Germanane FieldEffect Transistors. 2D Mater. 2017, 4 (2), 021009.

[22] Zandvliet, H. J. W. The Ge(001) Surface. Phys. Rep. 2003, 388 (1), 1-40.

[23] Bampoulis, P.; Zhang, L.; Safaei, A.; Gastel, R. van; Poelsema, B.; Zandvliet, H. J. W. Germanene Termination of $\mathrm{Ge}_{2} \mathrm{Pt}$ Crystals on Ge(110). J. Phys. Condens. Matter 2014, 26 (44), 442001.

[24] Zhang, L.; Bampoulis, P.; van Houselt, A.; Zandvliet, H. J. W. Two-Dimensional Dirac Signature of Germanene. Appl. Phys. Lett. 2015, 107 (11), 111605.

[25] Bampoulis, P.; van Bremen, R.; Yao, Q.; Poelsema, B.; Zandvliet, H. J. W.; Sotthewes, K. Defect Dominated Charge Transport and Fermi Level Pinning in $\mathrm{MoS}_{2}$ /Metal Contacts. ACS Appl. Mater. Interfaces 2017, 9 (22), 19278-19286.

[26] Addou, R.; Colombo, L.; Wallace, R. M. Surface Defects on Natural MoS 2. ACS Appl. Mater. Interfaces 2015, 7 (22), 11921-11929.

[27] Liu, C.-C.; Feng, W.; Yao, Y. Quantum Spin Hall Effect in Silicene and TwoDimensional Germanium. Phys. Rev. Lett. 2011, 107 (7), 076802.

[28] Liu, C.-C.; Jiang, H.; Yao, Y. Low-Energy Effective Hamiltonian Involving Spin-Orbit Coupling in Silicene and Two-Dimensional Germanium and Tin. Phys. Rev. B 2011, 84 (19), 195430.

[29] Das Sarma, S.; Adam, S.; Hwang, E. H.; Rossi, E. Electronic Transport in TwoDimensional Graphene. Rev. Mod. Phys. 2011, 83 (2), 407-470. 
[30] Samaddar, S.; Yudhistira, I.; Adam, S.; Courtois, H.; Winkelmann, C. B. Charge Puddles in Graphene near the Dirac Point. Phys. Rev. Lett. 2016, 116 (12), 126804.

[31] Katsnelson, M. I. Nonlinear Screening of Charge Impurities in Graphene. Phys. Rev. B 2006, 74 (20), 201401.

[32] John, R.; Merlin, B. Optical Properties of Graphene, Silicene, Germanene, and Stanene from IR to Far UV - A First Principles Study. J. Phys. Chem. Solids 2017, 110, 307-315.

[33] Ezawa, M. Monolayer Topological Insulators: Silicene, Germanene, and Stanene. J. Phys. Soc. Jpn. 2015, 84 (12), 121003.

[34] Zhang, Y.; Brar, V. W.; Wang, F.; Girit, C.; Yayon, Y.; Panlasigui, M.; Zettl, A.; Crommie, M. F. Giant Phonon-Induced Conductance in Scanning Tunnelling Spectroscopy of Gate-Tunable Graphene. Nat. Phys. 2008, 4 (8), 627-630.

[35] Wang, X.-Q.; Li, H.-D.; Wang, J.-T. Induced Ferromagnetism in One-Side Semihydrogenated Silicene and Germanene. Phys. Chem. Chem. Phys. 2012, 14 (9), 30313036.

[36] Nijamudheen, A.; Bhattacharjee, R.; Choudhury, S.; Datta, A. Electronic and Chemical Properties of Germanene: The Crucial Role of Buckling. J. Phys. Chem. C 2015, 119 (7), 3802-3809.

[37] Qiu, J.; Fu, H.; Xu, Y.; Oreshkin, A. I.; Shao, T.; Li, H.; Meng, S.; Chen, L.; Wu, K. Ordered and Reversible Hydrogenation of Silicene. Phys. Rev. Lett. 2015, 114 (12), 126101.

[38] Qiu, J.; Fu, H.; Xu, Y.; Zhou, Q.; Meng, S.; Li, H.; Chen, L.; Wu, K. From Silicene to Half-Silicane by Hydrogenation. ACS Nano 2015, 9 (11), 11192-11199. 


\section{Impact of defects and air-stability on $\mathrm{HfSe}_{2}$}

\subsection{Introduction}

Since the first single-layer $\mathrm{MoS}_{2}$ transistor was fabricated and characterized in $2011,{ }^{[1]}$ it has triggered many scientists to search for transition metal dichalcogenides (TMDs)-based applications. Two-dimensional TMDs have been widely studied because of their appealing physical (1T or $2 \mathrm{H}$ phase) and electrical properties (ranging from metallic to half-metallic to semiconducting and even superconducting). ${ }^{[2-4]}$ For instance, $\mathrm{MoS}_{2}$ has a thickness dependent bandgap, with a transition of an indirect to direct bandgap in case that the thickness is reduced to a single layer. ${ }^{[5]}$ In addition, superconductivity has been observed in both bulk $1 \mathrm{~T}-\mathrm{MoS}_{2}{ }^{[6]}$ and pristine $2 \mathrm{H}_{\mathrm{a}}-\mathrm{MoS}_{2}{ }^{[7]}$. However, compared to Mo or W chalcogenides, HfSe 2 , has not been received that much of attention. Single-layer $\mathrm{HfSe}_{2}$ is a material with an octahedral structure with an indirect band gap of $\sim 1 \mathrm{eV},{ }^{[8-10]}$ which makes it an appealing candidate for semiconductor device applications.

$\mathrm{HfSe}_{2}$-based prototype transistors have been realized and investigated by several groups. ${ }^{[9,11-13]}$ A high on/off current ratio, exceeding $10^{6}$, was found, satisfying the requirement for effective switching in digital logic transistors. ${ }^{[11]}$ The current density of a trilayer $\mathrm{HfSe}_{2}$ was up to 30 $\mu \mathrm{A} / \mu \mathrm{m} .{ }^{[9]}$ Moreover, a phototransistor based on $\mathrm{HfSe}_{2}$ has superior optoelectronic properties with an ultrafast response time and high photocurrent, which is comparable with other TMDs phototransistors. ${ }^{[12]}$ However, its carrier mobility is rather low, ranging from $\sim 0.3$ to $\sim 6.5$ $\mathrm{cm}^{2} \cdot \mathrm{V}^{-1} \cdot \mathrm{S}^{-1}$, i.e., three orders of magnitude lower than the predicted value of $\sim 3500 \mathrm{~cm}^{2} \cdot \mathrm{V}^{-1} \cdot \mathrm{S}^{-}$ ${ }^{1}$ at room temperature. ${ }^{[14]}$

Besides device optimization, such as the inclusion of dielectric layers and the selection of proper metal contacts, the quality of the material should be also improved, in order to obtain properly operating devices. It is known that TMD materials suffer from a large number of intrinsic defects, located either in the transition metal layer or the chalcogenide layer. ${ }^{[15-17]}$ Moreover, the air stability of $\mathrm{HfSe}_{2}$ needs to be investigated too. These key issues, i.e. intrinsic defects and air stability, have to be considered in order to properly evaluate the application prospects of $\mathrm{HfSe}_{2}$.

Defects often play a significant role in determining the electronic behavior in TMDs. ${ }^{[18-23]}$ Intrinsic defects dominate the contact resistance between metal contacts and TMDs ${ }^{[19]}$ and can act as scattering centers ${ }^{[20]}$, degrading the charge carrier mobility. Defects are also expected to 
dominate the surface conductivity of $\mathrm{HfSe}_{2}$ leading to the very low carrier mobilities. It is therefore essential to scrutinize the structural and electronic characteristics of $\mathrm{HfSe}_{2}$ and explore the reasons of the poor device performance. We have used scanning tunneling microscopy and spectroscopy (STM/STS) to study variations in the structure and electronic local density of states (LDOS) of the HfSe 2 surface. We have found several types of defects located either in the uppermost Se layer or Hf layer. Furthermore, we have used conductive atomic force microscopy (C-AFM) to measure the influence of these defects on the Schottky barrier height formed with different metal contacts and possible partial Fermi level pinning effects, following the same approach as in ref [21] for $\mathrm{MoS}_{2}$.

In addition, $\mathrm{HfSe}_{2}$ crystals are not stable against oxidation at ambient conditions, implying this air-sensitive $\mathrm{HfSe}_{2}$ surface is detrimental for many applications. Gioele et al. ${ }^{[24]}$ found a preferential reaction of oxygen with the Hf atoms rather than with the Se atoms, leading to the formation of a $\mathrm{HfO}_{2}$ layer. In principle the poor air stability of $\mathrm{HfSe}_{2}$ should limit its application in electronic devices. However, $\mathrm{HfO}_{2}$ is a high- $\kappa$ dielectric oxide, which has been frequently used as a top gate layer for field-effect transistors. ${ }^{[1,25]}$ In particular, native $\mathrm{HfO}_{2}$ layer can effectively suppress the interfacial charge trap states at the $\mathrm{HfSe} / \mathrm{HfO}_{2}$ interface, which is very beneficial for device performance. ${ }^{[9]}$ Therefore, it is important to properly understand the oxidation of $\mathrm{HfSe}_{2}$. Unlike earlier Raman and XPS studies ${ }^{[8,13,24]}$, the combination of STM and C-AFM allow us to study the oxidation dynamics and evolution of the electrical properties of $\mathrm{HfSe}_{2}$ with a high spatial resolution.

\subsection{Experimental Details}

The $\mathrm{HfSe}_{2}$ crystal was purchased from HQ Graphene (Groningen, The Netherlands). The samples were cleaned by mechanical exfoliation and subsequently transferred to vacuum system as soon as possible for STM and C-AFM measurements. All the STM/STS data were obtained by room temperature scanning tunneling microscopy (Omicron STM1). The base pressure of the ultra-high vacuum system is $3 \times 10^{-11}$ mbar. In order to obtain $I(V)$ signals by AFM, we applied the bias voltages between the conductive tip (grounded) and $\mathrm{HfSe}_{2}$ surface to read out the current values. Different conductive tips, including the Pt tips, the PtSi tips and the n-type Si tips, were used for the measurements. The resonance frequency is $4.5,15$, and 160 $\mathrm{kHz}$ for the Pt, PtSi, and n-type Si tips, respectively. 


\subsection{Surface topography and local electronic properties}

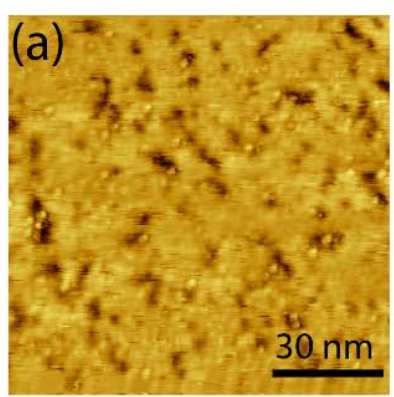

(e)
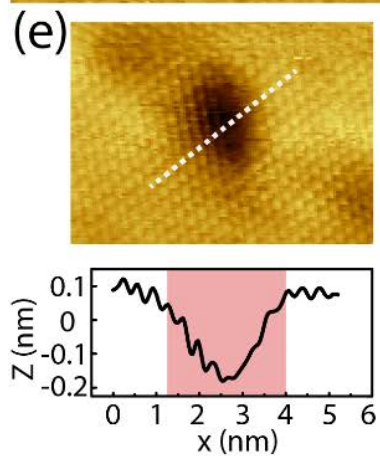
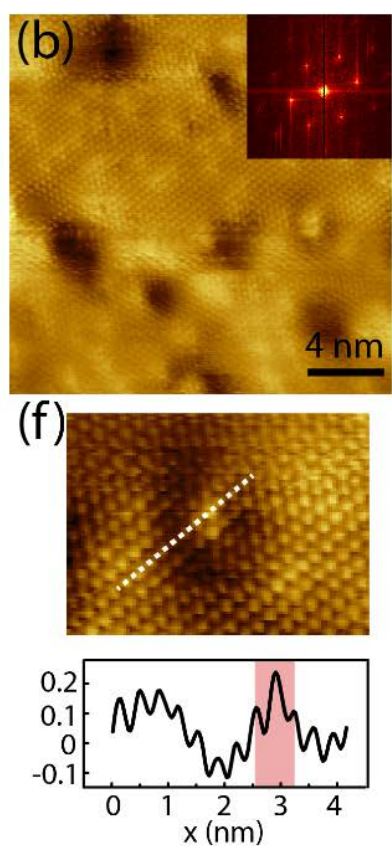

(c) top view

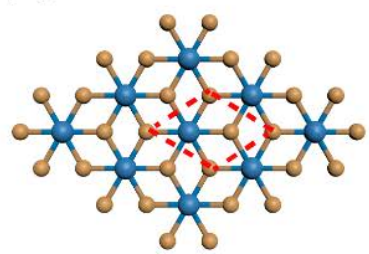

(d) side view

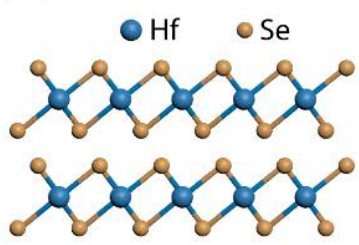

(g)
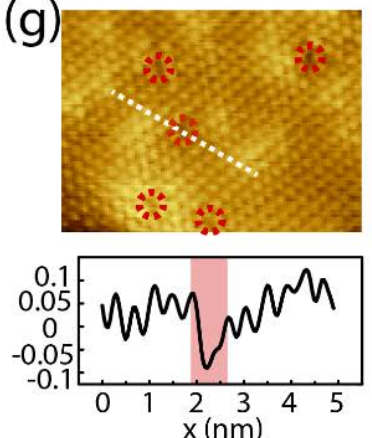
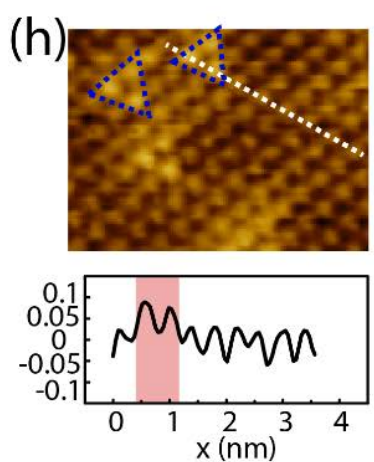

Figure 6.1: (a) Large-scale STM topography image on the HfSe 2 crystal $\left(100 \mathrm{~nm} \times 100 \mathrm{~nm}\right.$; $I_{\mathrm{t}}=$ $\left.0.6 \mathrm{nA}, V_{\mathrm{s}}=-0.3 \mathrm{~V}\right)$. (b) An atomically resolved image of the HfSe 2 surface $\left(20 \mathrm{~nm} \times 20 \mathrm{~nm} ; I_{\mathrm{t}}=\right.$ $0.6 \mathrm{nA}, V_{\mathrm{s}}=-0.4 \mathrm{~V}$ ). The inset is the corresponding FFT image. (c) Top view, and (d) side view sketches of a bilayer HfSe 2 . (e-h) Atomic scale STM images and line scans of defects on $\mathrm{HfSe}_{2}$ $\left(I_{\mathrm{t}}=0.6 \mathrm{nA}, V_{\mathrm{s}}=-0.4 \mathrm{~V}\right)$.

Because of the poor air instability of $\mathrm{HfSe}_{2}$, the mechanical exfoliation was done in-situ in the load lock of the STM system at a base pressure of $1 \times 10^{-8}$ mbar. The freshly cleaved sample was then transferred to the main chamber of the STM for imaging. Figures 6.1a shows a large-scale STM topography image of the freshly cleaved $\mathrm{HfSe}_{2}$ surface. It is clear that the surface of $\mathrm{HfSe}_{2}$ is inhomogeneous, displaying a large number of defects. The density of these defects across the whole sample is about $9 \times 10^{-11} \mathrm{~cm}^{-2}$, which is substantially higher than the defect densities of $\mathrm{MoS}_{2}$ or WSe $2,{ }^{[26,27]}$ see Figure 6.2. 

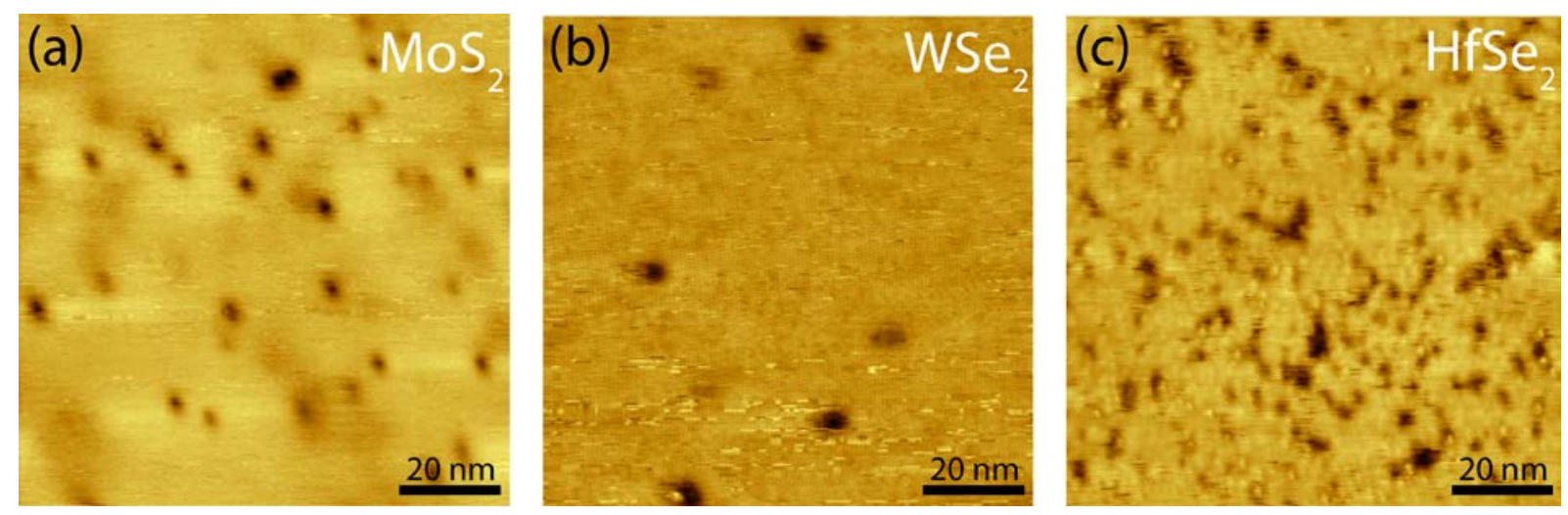

Figure 6.2: STM topography images of three different TMD surfaces, obtained by the same scanning tunneling microscopy settings. The image size is $100 \mathrm{~nm} \times 100 \mathrm{~nm}$, sample bias voltage is $-0.3 \mathrm{~V}$ and tunnel current is $0.6 \mathrm{nA}$.

Such a high density of defects makes it very difficult to find nanometer sized defect-free regions. By zooming in at parts where the surface structure is still intact, we have acquired the atomic lattice of the surface, as shown in Figure 6.1b. The lattice constant, as extracted from the images is $0.378 \mathrm{~nm}$ (see the inset in Figure 6.1b for a fast Fourier transform). This value is in good agreement with the previously reported lattice constant. ${ }^{[28]}$ Figure $6.1 \mathrm{c}$ and $6.1 \mathrm{~d}$ shows the structural model of bilayer $\mathrm{HfSe}_{2}$. The interlayer distance of the $\mathrm{HfSe}_{2}$ crystal is $\sim 0.614 \mathrm{~nm} .^{\text {[29] }}$ The single-layer $\mathrm{HfSe}_{2}$ is constituted of a top-Se, center-Hf, and bottom-Se atom layer, respectively. These three atom layers acquire an octahedral configuration. In our atomically resolved STM images only the top-Se atom layer is observed. The unit cell formed by the nearest four neighbor Se atoms corresponds to the red dashed contour marked in the top view of the structural model. 
(a)

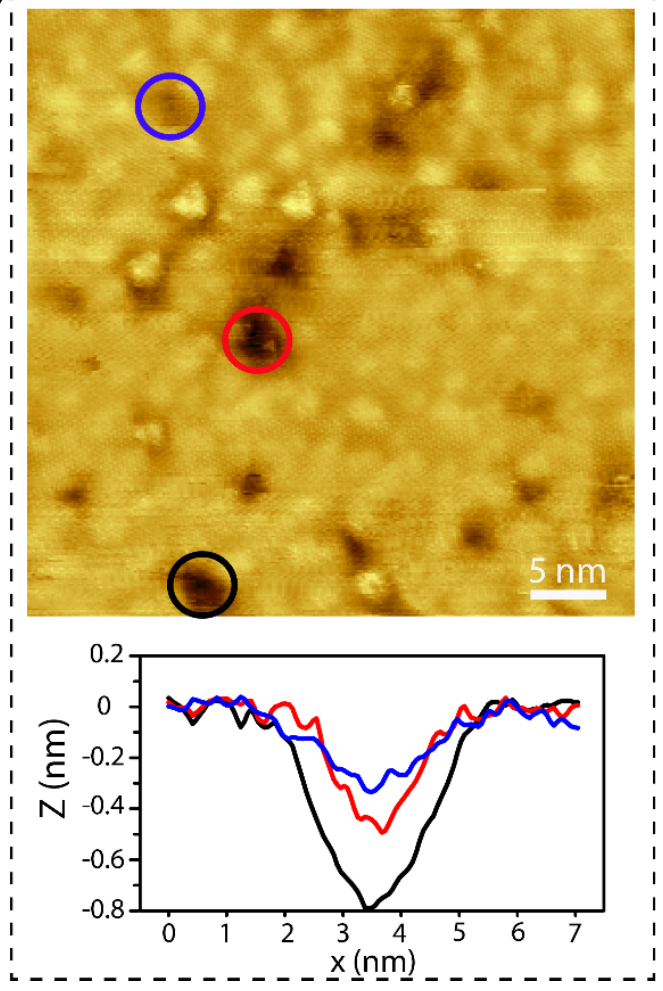

(b)
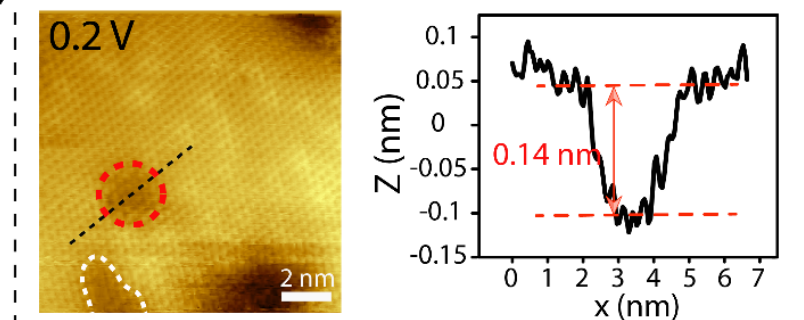

$0.05 \mathrm{~V}$

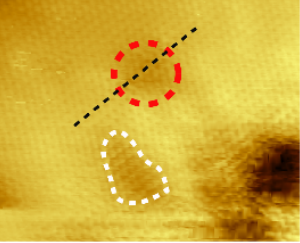

$-0.1 \mathrm{~V}$
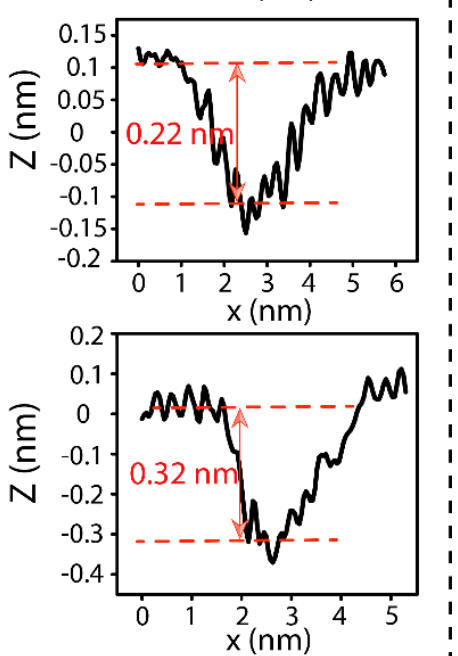

Figure 6.3: Height of the depression for dark defects. Panel (a): three different positions, marked by the blue, red, and black rings. The heights are $0.3,0.5$, and $0.8 \mathrm{~nm}$, respectively. Panel (b): The exact height of the same dim dark defect marked by the dashed red contour, depends on the sample bias voltage.

The most abundant defects are shown in Figure 6.1e-f, which are dark defects, bright defects, and atom vacancies, respectively. The dark defect in Figure 6.1e has a depth measured by the line profile of around $0.3 \mathrm{~nm}$. Depending on the defect position and sample bias voltage, the height of this depression varies from around $0.1 \mathrm{~nm}$ to $0.8 \mathrm{~nm}$, as described in detail in the Figure 6.3. It is worth noting that three types of dark defects have been identified based on their topographical features: 1 ) a very dark hole-like defect with the depression depth of $\geq 0.7 \mathrm{~nm}$, 2) a less dark defect with the depression depth ranging from $0.5 \mathrm{~nm}$ to $0.7 \mathrm{~nm}$ and 3) a dim dark defect with a visible continuous lattice periodicity and the depression depth of $\leq 0.5 \mathrm{~nm}$, see Figure 6.4). The third type of defects (dim dark) have a density of $5 \times 10^{11} \mathrm{~cm}^{-2}$. Defects with a similar appearance are also reported to exist on other TMD crystals, either on pristine surfaces or after ion bombardment. ${ }^{[26,27,30-32]}$ Since our HfSe 2 sample was only treated with scotch-tape cleavage before scanning, the ion bombardment induced defects can be ignored here. The dark defects have been interpreted to be caused by poor growth conditions. Its appearance is the 
result of electron depletion caused by the Coulomb repulsive potential around the Se atom complex or the acceptor impurity near the surface. ${ }^{[27,33]}$ The continuation of the lattice periodicity suggests that this type of feature is due to the absence of a Se atom.
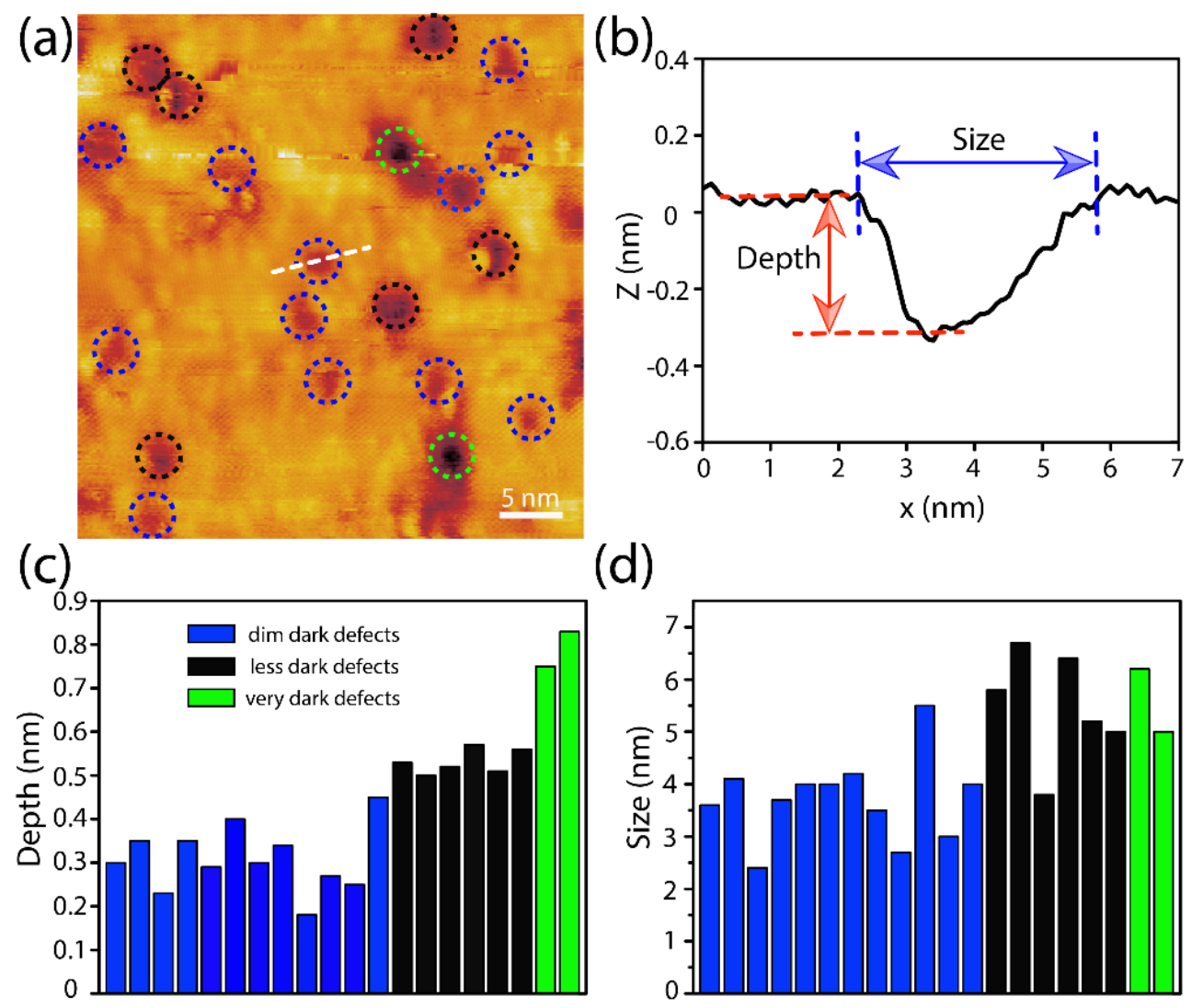

(d)

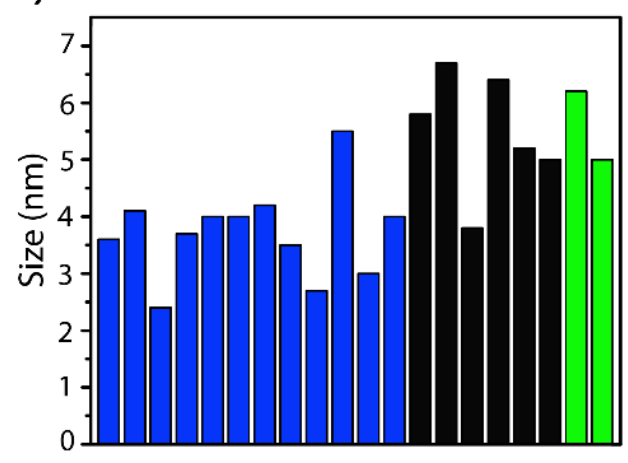

Figure 6.4: The three types of dark defects. (a) STM topography image of $\mathrm{HfSe}_{2}$ surface, the image size is $40 \mathrm{~nm} \times 40 \mathrm{~nm}$, sample bias voltage is $-0.4 \mathrm{~V}$ and tunnel current is $0.6 \mathrm{nA}$. The three types of defects, marked by the blue, black, and green contours, are corresponding to the dim dark defect, less dark defects and very dark defects, respectively; (b) The depth and size of the defect marked by the dashed white line; (c) Depth of the dark defects from (a); (d) Size of the dark defects from (a).

In addition to these abundant dark defects, we have found several other types of defects on the surface. Figure 6.1f presents a hillock-like defect with a density of $\sim 2.5 \times 10^{11} \mathrm{~cm}^{-2}$, probably caused by an adatom. The adatom could be for instance a Se atom, or another impurity atom such as $\mathrm{Li}, \mathrm{Na}, \mathrm{K}$ and $\mathrm{Re} .^{[32,34]}$ Moreover, several single-atom vacancies are also found, as shown in Figure 1g. The corresponding cross section reveals the absence of an atom in the lattice. Such single atom vacancy has a density of $\sim 0.5 \times 10^{11} \mathrm{~cm}^{-2}$. We also report a new type of defect with a triangular shape, shown in Figure 1 h. It has a similar appearance as the $3 \times 3$ 
charge density wave superlattice observed in $\mathrm{NbSe}_{2} \cdot{ }^{[35]}$ Since the charge density wave transition temperature of $\mathrm{HfSe}_{2}$ is rather low we can exclude this interpretation here. It has been shown that $\mathrm{HfSe}_{2}$ is a suitable host material for extrinsic dopant atoms, ${ }^{[36]}$ suggesting that the bright defects might be a result of the intercalation of impurities.

(a)

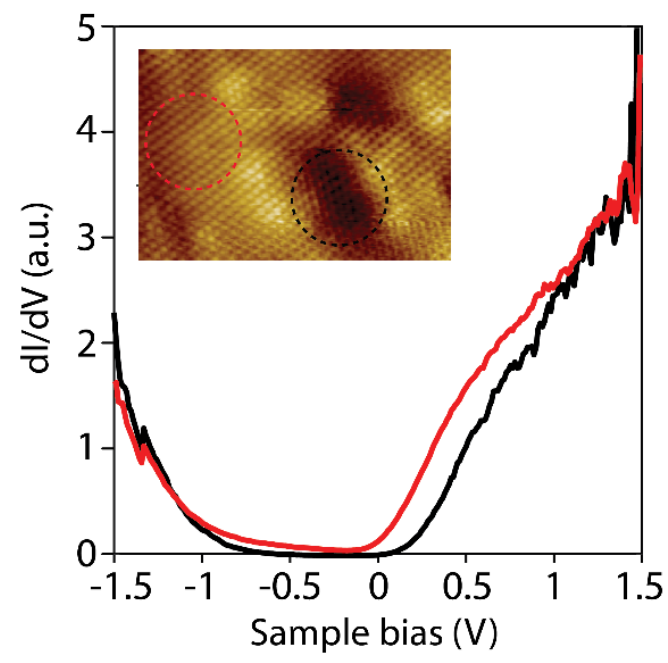

(b)

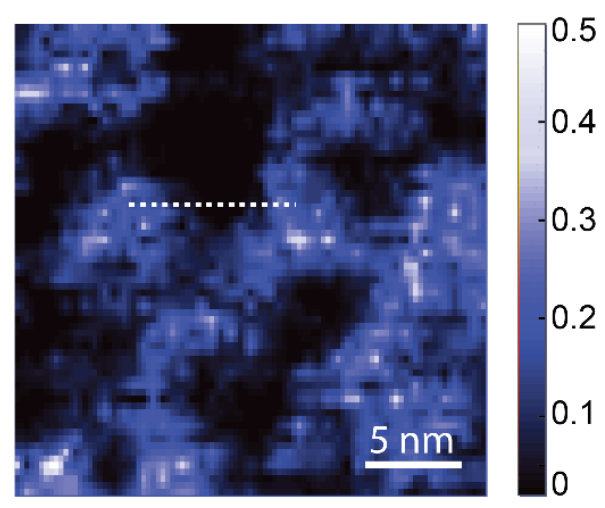

(c)

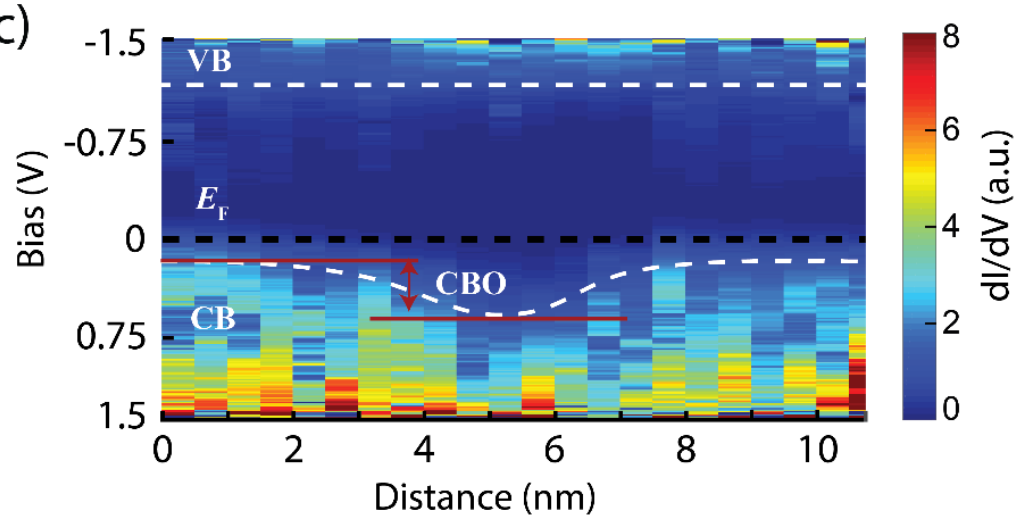

Figure 6.5: Scanning tunneling spectroscopy results of the HfSe 2 surface (a) $d I / d V$ spectra at different regions, Inset: STM image showing the locations where the spectroscopy data were taken from. (b) $d I / d V$ map of $\mathrm{HfSe}_{2}\left(100 \mathrm{~nm} \times 100 \mathrm{~nm} ; I_{\mathrm{t}}=0.6 \mathrm{nA}, V_{\mathrm{s}}=-0.3 \mathrm{~V}\right)$. (c) Colourcoded rendering of the real space imaging of the band profile plotted in terms of $d I / d V$ taken along the dashed white line in Figure 2b.

In the following section, we investigate the influence of the aforementioned defects on the local density of states of the $\mathrm{HfSe}_{2}$ surface using scanning tunneling spectroscopy (STS). Figure 6.5a shows the local tunneling conductance $(d I / d V)$ spectra taken at the red and black dashed circles, which correspond to the dim dark defect and the pristine region, respectively. The $d I / d V$ spectra, which are proportional to the electronic local density of states (LDOS) near the Fermi level for 
small sample biases, are obtained by numerical differentiating 3600 current-voltage curves recorded in a $60 \times 60$ grid. Both recorded curves exhibit an n-type semiconducting behavior, in good agreement with previous STM and electrical transport measurements. ${ }^{[8,11-12]}$ The Fermi level is much closer to the conduction band (CB) than to the valence band (VB) because of the strong doping from the Se atom vacancies in the crystal. The sharp increases in the $d I / d V$ spectra correspond to the CB and VB band edges. The bandgap as extracted from these curves is about $1 \mathrm{eV}$ for the pristine $\mathrm{HfSe}_{2}$ surface and $1.25 \mathrm{eV}$ for the dim dark defects, respectively. In addition, the $d I / d V$ map in Figure 6.5b illustrates the large fluctuation of the LDOS of $\mathrm{HfSe}_{2}$, induced by the presence of the high density of intrinsic defects on $\mathrm{HfSe}_{2}$ surface. The $d I / d V$ spectra along the dashed white line in Figure 6.5b are taken to determine the real space band profiles. As shown in Figure 6.5c, there is a conduction band offset (CBO) between the conduction band minima of the defect and the pristine surface that amounts to $\sim 0.2$ $\mathrm{eV}$. In principle, $\mathrm{S}$ or Se atom vacancies in TMDs can lead to enhancement of the LDOS in the conduction band because of the broken covalent bonds, leading to a reduction of the CBM. ${ }^{[37 \text {, }}$ ${ }^{38]}$ In contrast, we observe an upward shift of the CBM. The atomically resolved STM topography images of the dim dark defects (shown in Figure 6.1e) show an intact lattice periodicity, indicating that these defects are buried under the surface. Therefore, the possible mechanism for the shift of the CBM might involve substitutional dopants, like Mo, W or Sn atoms. The presence of these substitutional atoms could be caused by the crystal growth process.. The brighter LDOS signal in Figure 6.5b is most probably related to the presence of triangular defects, however their considerable smaller size does not allow for accurately capturing their influence on the surface.

Spatial maps of the LDOS recorded at various energies on the same $\mathrm{HfSe}_{2}$ surface location as discussed in Figure 6.5b, are shown in Figure 6.6. These $d I / d V$ maps are recorded simultaneously with the topographic image. The dark (red arrows) and bright (green arrows) regions correspond to the dark defects and the pristine areas, respectively. In the vicinity of the Fermi level ( $-0.15 \mathrm{eV} \leq E \leq 0.15 \mathrm{eV})$, large inhomogeneities of the LDOS are clearly observed. The pristine $\mathrm{HfSe}_{2}$ has a higher conductance, which is in accordance with the $d I / d V$ curves shown in Figure 6.5a. For energies is far away from Fermi level, the distribution of the LDOS becomes more and more uniform. 

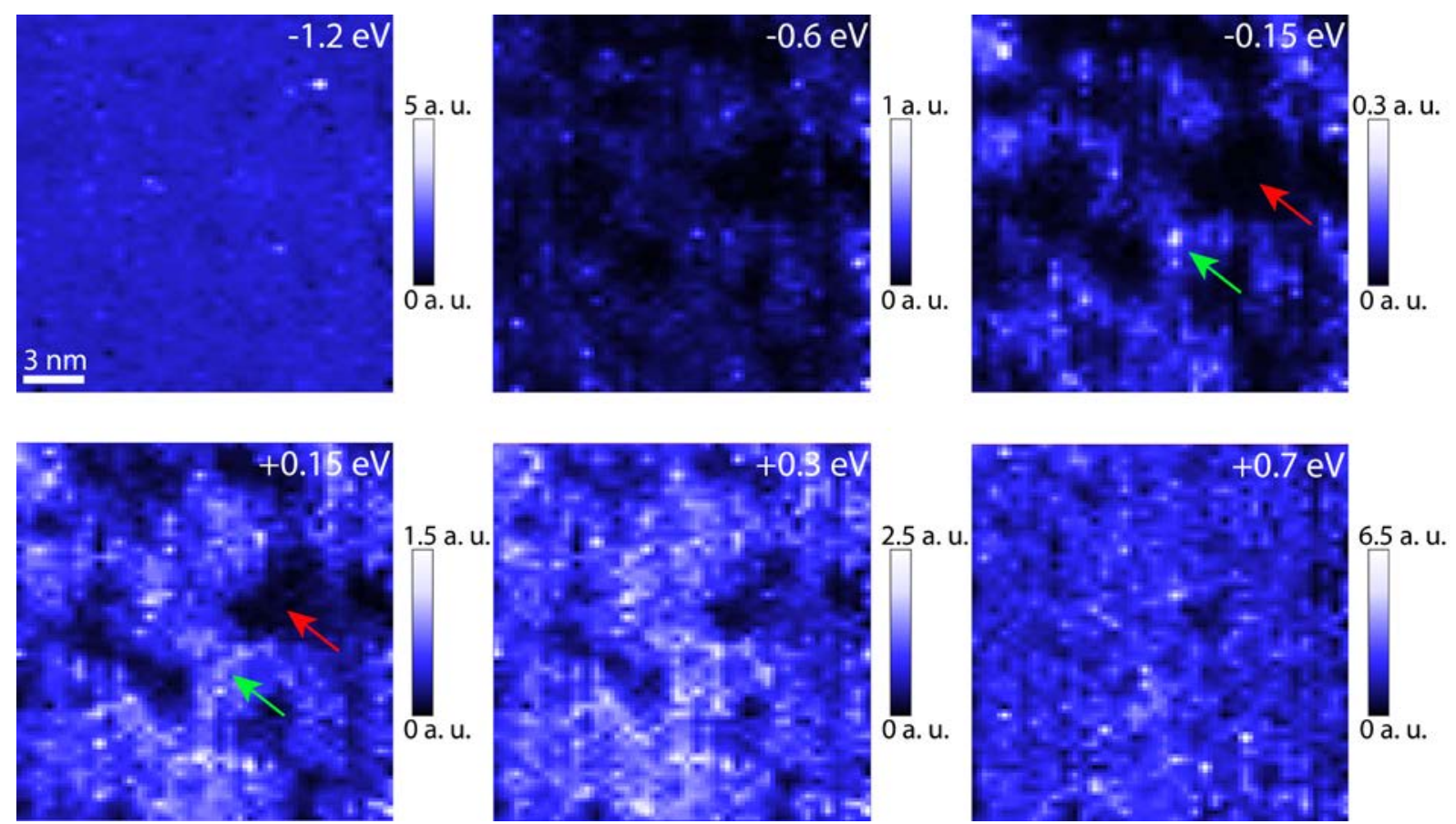

Figure 6.6: Spatially resolved density-of-state maps obtained on $\mathrm{HfSe}_{2}$ surface at different energies $\left(20 \mathrm{~nm} \times 20 \mathrm{~nm}, I_{\mathrm{t}}=0.5 \mathrm{nA}\right)$, taken at $-1.2 \mathrm{~V},-0.6 \mathrm{~V},-0.15 \mathrm{~V},+0.15 \mathrm{~V},+0.3 \mathrm{~V}$ and +0.7 $\mathrm{V}$, respectively.

\subsection{Schottky barrier height and Fermi level pinning of the $\mathrm{HfSe}_{2}$ crystal}

Owing to the moderate bandgap of around $1 \mathrm{eV}$, which is comparable to silicon, HfSe 2 -based field effect transistors have been investigated. However, the mobility obtained in transport measurements is much lower than the theoretically predicted one. ${ }^{[9,11,13]}$ The following reasons for the low charge carrier mobility have been put forward. Firstly, the high anisotropic electrical resistivity of $\mathrm{HfSe}_{2}$ can suppress the vertical charge transport in the Ohmic regime. Secondly, the barrier at non-ideal Ohmic contacts induces large contact resistances and further limits the mobility. Thirdly, the environmental instability of $\mathrm{HfSe}_{2}$ can cause degradation of the device. In addition, the existence of possible interfacial scattering and interlayer resistance could be also responsible for the low charge carrier mobility. Although a slow decay time of photocurrent in Au-contacted $\mathrm{HfSe}_{2}$ phototransistor was shown to be correlated to defects and charge impurity states ${ }^{[12]}$ the effect of defects on the charge transport of $\mathrm{HfSe}_{2}$ has not yet been studied. In our STM images we have confirmed the high-density of these defects and impurities. The strong influence of the dark defects in the LDOS indicates local variations in electrical contacts. 


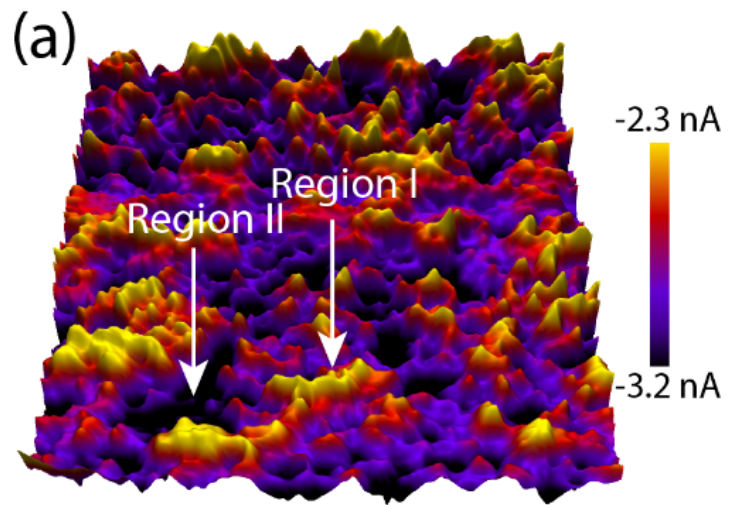

(c)

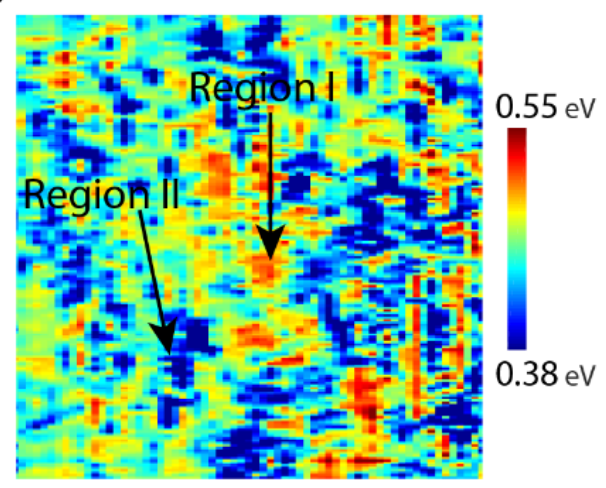

(b)

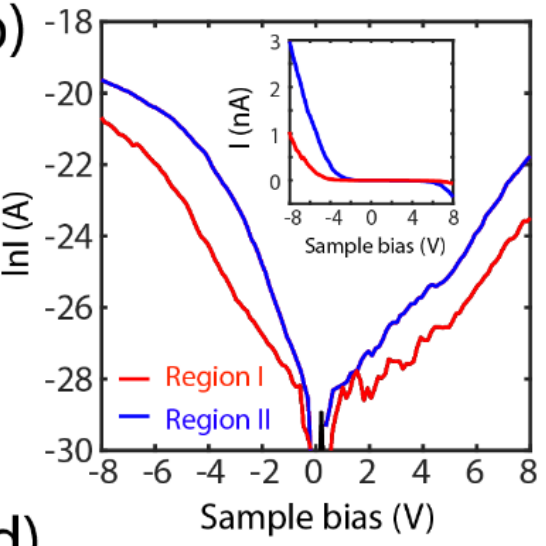

(d)

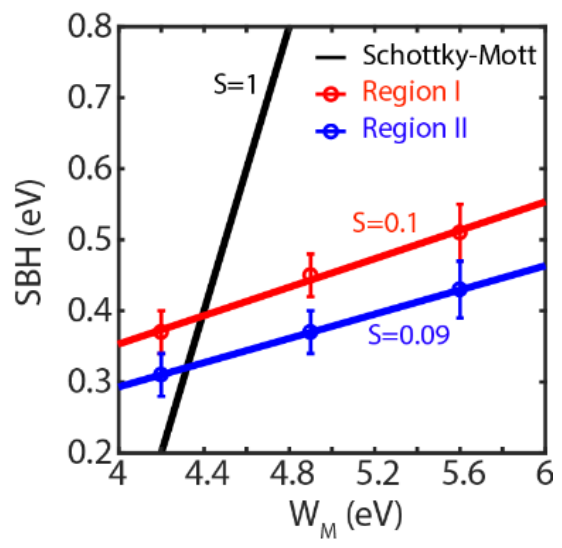

Figure 6.7: (a) A current image $(600 \mathrm{~nm} \times 600 \mathrm{~nm})$ of the $\mathrm{HfSe}_{2}$ recorded with C-AFM at a sample bias of $4.7 \mathrm{~V}$. A highly doped Si tip (work function $4.2 \mathrm{eV}$ ) has been used. (b) The semi-logarithmic contact $I(V)$ curves for region I and region II, as indicated in panel (a). Inset: the corresponding contact $I(V)$ curves. (c) The extracted Schottky barrier height (SBH) map $(200 \mathrm{~nm} \times 200 \mathrm{~nm})$ of the surface extracted from $I(V)$ curves, as shown in panel (b). The apparent SBH variations are attributed to the presence of defects on the sample. (d) The Schottky barrier heights of region I (red) and region II (blue) as a function of the tip's work functions( $\left.W_{\mathrm{M}}\right)$. The pinning factor $S$ is extracted using eq 6 . The black line refers to the standard Schottky-Mott rule (see eq 5).

Since a C-AFM tip can be used as a metal nanocontact to measure simultaneously the topography image and $I(V)$ curves, it is possible to make a metal-semiconductor (tip-HfSe 2 ) junction and obtain information on the variation in electrical resistance and contact properties induced by defects on the HfSe 2 . Figure 6.7a shows the current map recorded with C-AFM using a highly n-doped Si tip with a work function of about $4.2 \mathrm{eV}$ and a radius of curvature of $2 \mathrm{~nm}$. The inhomogeneous current distribution is consistent with STM topography images. 
Moreover, voltage-dependent current spectra are recorded in $128 \times 128$ grid fashion, the average curves recorded in region I and region II are shown in Figure 6.7b. We find different charge transport characteristics in region I (red line) and region II (blue line), with region II being much more conductive than region I, especially in the negative sample bias regime (see inset in Figure 6.7b). Furthermore, the $I(V)$ traces reveal a rectifying behavior with an n-type Schottky barrier. The observation of an n-type Schottky barrier is reminiscent of the n-type doping of $\mathrm{HfSe}_{2}$ surface. In order to understand the difference between region I and region II, we have considered thermionic emission as the main transport process. The thermionic emission current $I$ is given by,

$$
I=I_{0}\left[\exp \left(\frac{q V}{\eta \kappa_{\mathrm{B}} T}\right)-1\right]
$$

with

$$
I_{0}=A A^{*} T^{2} \exp \left(-\frac{q \phi_{\mathrm{B}}}{\kappa_{\mathrm{B}} T}\right)
$$

where $I_{0}$ is the saturation current, $V$ is the applied bias voltage, $q$ is the electron charge, and $T$ is the sample temperature. A is the AFM tip contact area, calculated using the Hertz model. ${ }^{[39]}$ $\kappa_{\mathrm{B}}$ and $A^{*}$ are the Boltzmann constant and the Richardson constant, respectively. Then, the ideality factor $(\eta)$ and the Schottky barrier height $\left(\Phi_{\mathrm{B}}\right)$ are obtained by

and

$$
\eta=\frac{q}{\kappa_{\mathrm{B}}} \frac{d V}{d(\ln I)}
$$

The calculated Schottky barrier height map is shown in Figure 6.7c, two distinct regions matching region I and region II of the current image are observed, with region I having a larger $\Phi_{\mathrm{B}}$ than region II. In the map, the highest barrier height is about $0.55 \mathrm{eV}$, while the lowest one is around $0.38 \mathrm{eV}$. Combined with the STS results of Figure 6.5, we assign region I to be a defect-dominated area. In principle, the Schottky barrier height expected from the SchottkyMott rule should be $\sim 0.2 \mathrm{eV}$ according to the following equation:

$$
\Phi_{\mathrm{B}}=W_{m}-\chi
$$

The work function $W_{m}$ of the tip is around $4.2 \mathrm{eV}$, and the electron affinity $\chi$ of the $\mathrm{HfSe}_{2}$ is 4.0 $\mathrm{eV} \cdot{ }^{[8]}$ The measured barrier height is larger than the expected value by about $0.18-0.35 \mathrm{eV}$. We attribute this discrepancy to partial Fermi level pinning. The difference in the $\Phi_{\mathrm{B}}$ between region I and region II can be understood by the observed difference in the location of the CBM at the two regions (see the STM/STS measurements). The lower CBM (by about $0.2 \mathrm{eV}$ ) in 
region II leads to a smaller barrier height as compared to region I. Moreover, the ideality factors obtained in both regions are between 2-4, indicating that thermionic emission is not the sole contributor to the current, but tunnelling across the barrier also plays a role.

In order to investigate the partial Fermi level effect, we have investigated the charge transport characteristics for tips of different work function (PtSi of $4.8 \mathrm{eV}$ and Pt of $5.6 \mathrm{eV}$ ). The $\Phi_{\mathrm{B}}$ determined at the two regions for each tip are then plotted as a function of the work function, Figure 6.7d. The pinning factor is then quantified by the slope $S$ of the curve, and given by

$$
S=d \Phi_{\mathrm{B}} / d W_{m}
$$

Here, $S$ takes values between 0 and $1 . S=1$ indicates an unpinned interface and the SchottkyMott rule is fully applicable. For $S=0$, the $\Phi_{\mathrm{B}}$ is independent of the metal work function and the Fermi level is fully pinned. For $S$ between 0 and 1 partial Fermi level pinning occurs. Here we found that in region I $S$ is equal to 0.1 , while in region II $S$ is equal to 0.09 , both of these values signify a strong Fermi level pinning. A similar behavior has been also observed in metal/MoS${ }_{2}{ }^{[40,41]}$ Two mechanisms are suggested for the pinning effect in HfSe 2 . Firstly, distortion of the crystal surface to a large extent results in a notable charge redistribution at the interface between $\mathrm{HfSe}_{2}$ and the contact metal. This can reduce the width of Schottky barrier and enhance the orbital overlap, which could then cause the weak dependence of $W_{m}$ on $\Phi_{\mathrm{B}}$. Secondly, the Se atom vacancies on $\mathrm{HfSe}_{2}$ surface (see Figure 6.1g) that act as metallic defects can induce the formation of additional gap states, and subsequently strengthen the Fermi level pinning. 


\subsection{Effect of oxidation on $\mathrm{HfSe}_{2}$ Surface}
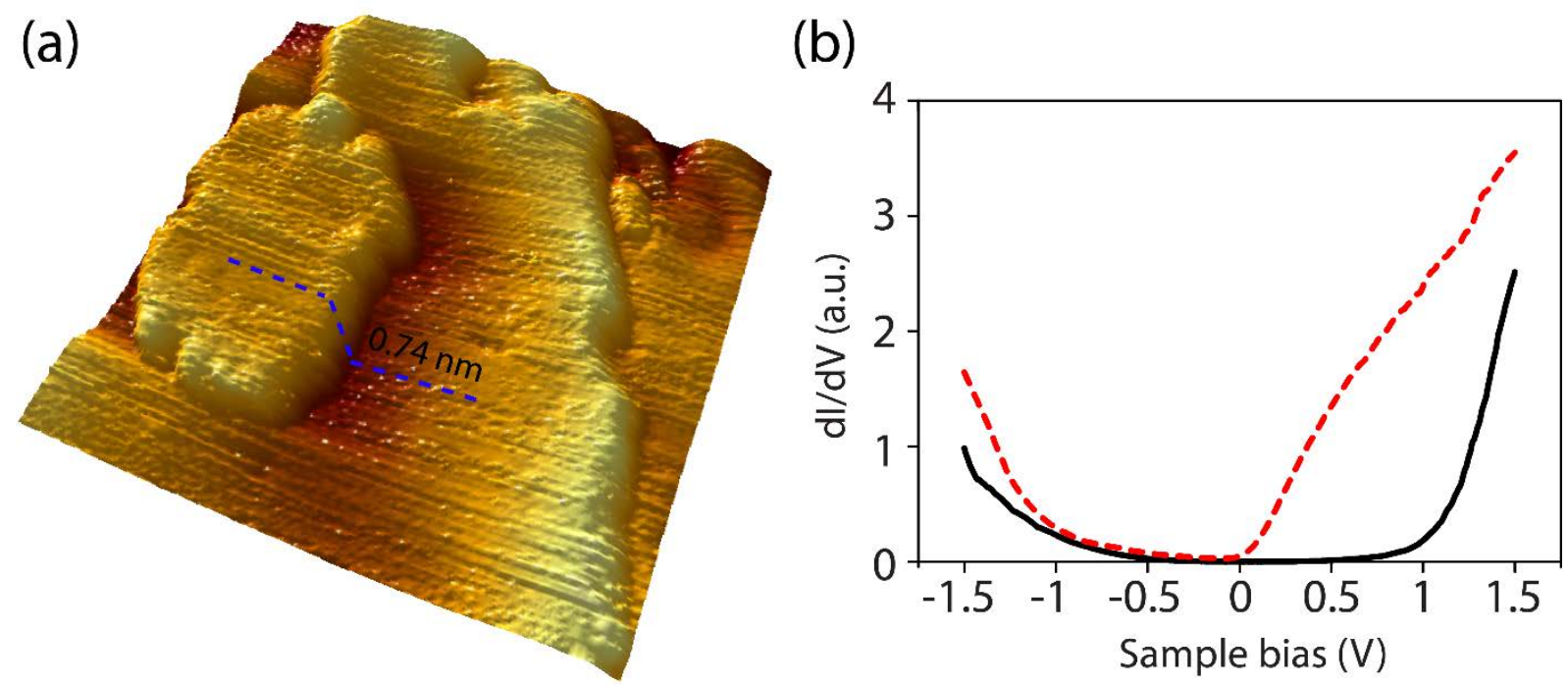

Figure 6.8. (a) Three dimensional (3D) image of HfSe 2 surface after air exposure for 2 days. The blue dashed line profiles the flake height, $I_{\mathrm{t}}=0.6 \mathrm{nA}, V_{\mathrm{s}}=-2.5 \mathrm{~V}$; (b) dI/dV spectra of the oxidized surface (black curve) and pristine $\mathrm{HfSe}_{2}$ (red dashed curve) measured with the same STM tip, $I_{\mathrm{t}}=0.4 \mathrm{nA}, V_{\mathrm{s}}=-1.5 \mathrm{~V}$.

The oxidation of the $\mathrm{HfSe}_{2}$ crystal was first investigated by STM. The 3D STM topography with a size of $25 \mathrm{~nm} \times 25 \mathrm{~nm}$ in Figure 6.8a presents the $\mathrm{HfSe}_{2}$ surface after 2 days of air exposure. Small flakes with thickness of $\sim 0.74 \mathrm{~nm}$ are readily found on the surface, i.e., a value about $0.13 \mathrm{~nm}$ larger than the interlayer distance of $\mathrm{HfSe}_{2}$. The surface of the material appears to be much rougher than the freshly cleaved surface. Because of this roughness, atomic resolution imaging of the crystal's lattice was not possible. The $d I / d V$ spectra recorded on the pristine and air-exposed $\mathrm{HfSe}_{2}$ surfaces are shown in Figure 6.8b. The bandgap of the air exposed surface as measured by STS is about $2 \mathrm{eV}$, which is almost two times larger than that of $\mathrm{HfSe}_{2}$. However, the expected bandgap of fully oxidized $\mathrm{HfO}_{2}$ is about $5.8 \mathrm{eV}{ }^{[42]}$ This difference suggests that the outermost $\mathrm{HfSe}_{2}$ layer is only partly oxidized and some of the HfSe bonds are still preserved. 
(a)

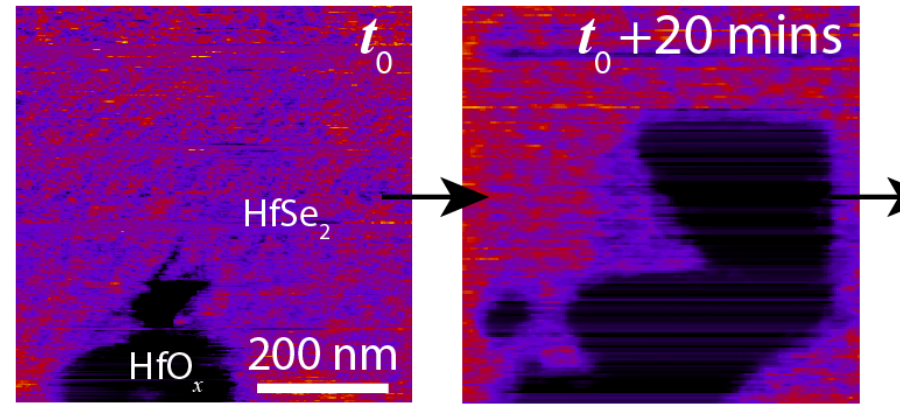

(b)

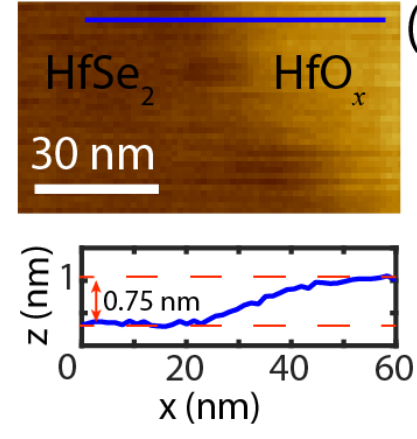

(c)

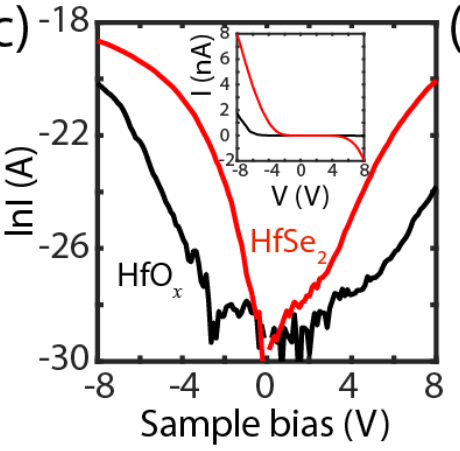

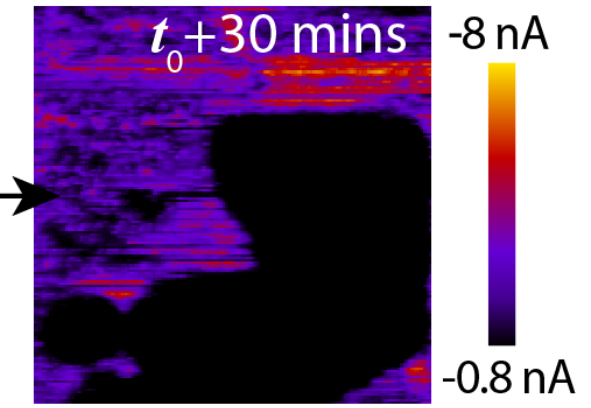

(d)

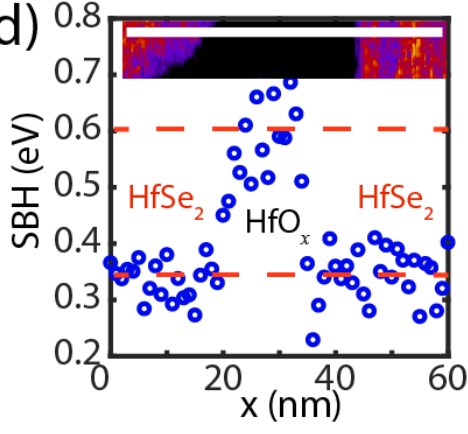

Figure 6.9. (a) The oxidation evolution of the $\mathrm{HfSe}_{2}$ as measured with C-AFM, which are taken at $t_{0}, t_{0}+20$ mins and $t_{0}+30$ mins, respectively. The sample bias is $4.7 \mathrm{~V}$ (b) AFM topography of the transition between an unoxidized to an oxidized region. The blue line shows the apparent height of $\mathrm{HfO}_{\mathrm{x}}$ layer. (c) The semi-logarithmic contact $\mathrm{I}(\mathrm{V})$ curves for $\mathrm{HfSe}_{2}$ and $\mathrm{HfO}_{\mathrm{x}}$. Inset: the corresponding contact I(V) curves. (d) Schottky barrier height cross section showing a $\mathrm{HfSe}_{2}-\mathrm{HfO}_{\mathrm{x}}-\mathrm{HfSe}_{2}$ transition.

The air stability of $\mathrm{HfSe}_{2}$ was also investigated with C-AFM. In contrast to the UHV measurements in the STM experiments, the C-AFM measurements were done in a very low humidity- $\mathrm{N}_{2}$ environment to exclude the influence from water. ${ }^{[43]}$ The sample, however, is still exposed to a certain amount of oxygen allowing us to study in-situ the oxidation dynamics of the uppermost $\mathrm{HfSe}_{2}$ layers. Figure 6.9a shows the time-dependent oxidation process of $\mathrm{HfSe}_{2}$ during a C-AFM measurement. At $t_{0}$, a small oxidized area was detected at the bottom side of the image by the distinct current intensity, which was one order of magnitude lower than that of the pristine surface. After 20 minutes of scanning of the same region, the oxidized part has spread along all directions, as shown in the second panel of Figure 6.9a. At $t_{0}+30$ minutes, the oxidized region occupies more than half of the image. The height of the $\mathrm{HfO}_{\mathrm{x}}$ flake was measured to be around $0.75 \mathrm{~nm}$, as shown in Figure 6.9b, in line with the STM result. The current-voltage characteristics averaged from several curves at the pristine and oxidized regions are shown in Figure 6.9c. As compared to the pristine $\mathrm{HfSe}_{2}$, the oxidized regions are significantly less conductive most probably due to the larger bandgap of these regions. The 
Schottky barrier height of this $\mathrm{HfO}_{\mathrm{x}}$ layer is determined to be $\sim 0.61 \mathrm{eV}$, i.e., about $44 \%$ larger than that of the unoxidized $\mathrm{HfSe}_{2}$ surface. More importantly, the ideality factor across these two regions has increased from approximately 4 at the unoxidized region to about 20 at the oxidized region. This huge increase in the ideality factor suggests that the current is not solely due to thermionic emission, but that also tunneling plays an important role. To this regard, the oxidized upper flake may act as a tunneling barrier. The measured structural and electrical variations confirm the air-sensitivity of $\mathrm{HfSe}_{2}$. $\mathrm{HfSe}_{2}$-based devices operated at ambient conditions will require the use of a protective coating layer in order to avoid oxidation. Another approach is to use the uppermost layer as a dielectric layer, complete oxidation of the uppermost layer is expected to slow down the oxidation of the underlying layers. To that extent new device architectures need to be considered to account for the effects observed here.

\subsection{Conclusions}

In summary, HfSe 2 crystal surface has been investigated in detail by STM and C-AFM. Atomically resolved STM images of the surface lattice structure demonstrates a high density of intrinsic defects, located both in the Se layer and Hf layer. The surface distortion and high density of defects indicate a very poor quality of the $\mathrm{HfSe}_{2}$ crystal, detrimental for many device applications. This poor quality most probably explains the low carrier mobilities found in $\mathrm{HfSe}_{2}$-based field effect transistors. A prominent defect with a dim dark appearance, localized at the Hf-layer with a density of $5 \times 10^{11} \mathrm{~cm}^{-2}$ locally increases the bandgap by about $25 \%$, changing it from $1 \mathrm{eV}$ to $1.25 \mathrm{eV}$. Moreover, the presence of defects reduces the local barrier height with metal contacts. Furthermore, the small dependence of the measured barrier height on the metal's work function indicates partial Fermi level pinning. We have also investigated the oxidation dynamics of $\mathrm{HfSe}_{2}$. After exposure of the crystal to ambient conditions, we find that oxidized layers grow fast and have a bandgap of $\sim 2 \mathrm{eV}$. The large increase of the Schottky barrier height and ideality factor of the oxidized parts when contacting with a metal tip, confirms our interpretation. Therefore, in order to obtain the predicted performance for $\mathrm{HfSe}_{2}-$ based electronic devices, the influence of defects and oxidation has to be well-controlled or eliminated. 


\section{Bibliography}

[1] Radisavljevic, B.; Radenovic, A.; Brivio, J.; Giacometti, V.; Kis, A. Single-Layer MoS2 Transistors. Nat. Nanotechnol. 2011, 6, 147-150.

[2] Butler, S. Z.; Hollen, S. M.; Cao, L.; Cui, Y.; Gupta, J. A.; Gutierrez, H. R.; Heinz, T. F.; Hong, S. S.; Huang, J.; Ismach, A. F., et al. Progress, Challenges, and Opportunities in TwoDimensional Materials Beyond Graphene. ACS Nano 2013, 7, 2898-2926.

[3] Jariwala, D.; Sangwan, V. K.; Lauhon, L. J.; Marks, T. J.; Hersam, M. C. Emerging Device Applications for Semiconducting Two-Dimensional Transition Metal Dichalcogenides. ACS Nano 2014, 8, 1102-1120.

[4] Duong, D. L.; Yun, S. J.; Lee, Y. H. van der Waals Layered Materials: Opportunities and Challenges. ACS Nano 2017, 11, 11803-11830.

[5] Splendiani, A.; Sun, L.; Zhang, Y.; Li, T.; Kim, J.; Chim, C.; Galli, G.; Wang, F. Emerging Photoluminescence in Monolayer MoS 2 . Nano Lett. 2010, 10, 1271-1275.

[6] Fang, Y.; Pan, J.; He, J.; Luo, R.; Wang, D.; Che, X.; Bu, K.; Zhao, W.; Liu, P.; Mu, G.; Zhang, H.; Lin, T.; Huang, F. Structure Re-Determination and Superconductivity Observation of Bulk 1T MoS 2 . Angew. Chem. 2018, 130, 1246-1249.

[7] Chi, Z.; Chen, X.; Yen, F.; Peng, F.; Zhou, Y.; Zhu, J.; Zhang, Y.; Liu, X.; Lin, C.; Chu, S.; Li, Y.; Zhao, J.; Kagayama, T.; Ma, Y.; Yang, Z. Superconductivity in Pristine $2 \mathrm{H}_{\mathrm{a}}-\mathrm{MoS}_{2}$ at Ultrahigh Pressure. Phys. Rev. Lett. 2018, 120, 037002.

[8] Yue, R.; Barton, A. T.; Zhu, H.; Azcatl, A.; Pena, L. F.; Wang, J.; Peng, X.; Lu, N.; Cheng, L.; Addou, R., et al. HfSe2 Thin Films: 2D Transition Metal Dichalcogenides Grown by Molecular Beam Epitaxy. ACS Nano 2015, 9, 474-480.

[9] Mleczko, M. J.; Zhang, C.; Lee, H. R.; Kuo, H.; Magyari-Kope, B.; Moore, R. G.; Shen, Z.; Fisher, I. R.; Nishi, Y.; Pop. HfSe 2 and $\mathrm{ZrSe}_{2}$ : Two-Dimensional Semiconductors with Native High-к Oxides. Sci. Adv. 2017, 3, e1700481. 
[10] Rasmussen, F. A.; Thygesen, K. S. Computational 2D Materials Database: Electronic Structure of Transition-Metal Dichalcogenides and Oxides. J. Phys. Chem. C 2015, 119, 13169-13183.

[11] Kang, M.; Rathi, S.; Lee, I.; Lim, D.; Wang, J.; Li, L.; Khan M. A.; Kim, G. Electrical Characterization of Multilayer HfSe 2 Field-Effect Transistors on $\mathrm{SiO}_{2}$ Substrate. Appl. Phys. Lett. 2015, 106, 143108.

[12] Yin, L.; Xu, K.; Wen, Y.; Wang, Z.; Huang, Y.; Wang, F.; Shifa, T. A.; Cheng, R.; Ma, H.; He, J. Ultrafast and Ultrasensitive Phototransistors Based on Few-Layered HfSe2. Appl. Phys. Lett. 2016, 109, 213105.

[13] Kang, M.; Rathi, S.; Lee, I.; Li, L.; Khan, M. A.; Lim, D.; Lee, Y.; Park, J.; Yun, S. J.; Youn, D.; Jun, C.; Kim, G. Tunable Electrical Properties of Multilayer $\mathrm{HfSe}_{2}$ Field Effect Transistors by Oxygen Plasma Treatment. Nanoscale 2017, 9, 1645-1652.

[14] Zhang, W.; Huang, Z.; Zhang, W.; Li, Y. Two-Dimensional Semiconductors with Possible High Room Temperature Mobility. Nano Res. 2014, 7, 1731-1737.

[15] Zhou, W.; Zou, X.; Najmaei, S.; Liu, Z.; Shi, Y.; Kong, J.; Lou, J.; Ajayan, P. M.; Yakobson, B. I.; Idrobo, J. Intrinsic Structural Defects in Monolayer Molybdenum Disulfide. Nano Lett., 2013, 13, 2615-2622.

[16] Liu, H.; Zheng, H.; Yang, F.; Jiao, L.; Chen, J.; Ho, W.; Gao, C.; Jia, J.; Xie, M. Line and Point Defects in $\mathrm{MoSe}_{2}$ Bilayer Studied by Scanning Tunneling Microscopy and Spectroscopy. ACS Nano 2015, 9, 6619-6625.

[17] Zhang, S.; Wang, C.; Li, M.; Huang, D.; Li, L.; Ji, W.; Wu, S. Defect Structure of Localized Excitons in a WSe 2 Monolayer. Phys. Rev. Lett. 2017, 119, 046101.

[18] Wu, Z.; Luo, Z.; Shen, Y.; Zhao, W.; Wang, W.; Nan, H.; Guo, X.; Sun, L.; Wang, X.; You, Y.; Ni, Z. Defects as a Factor Limiting Carrier Mobility in $\mathrm{WSe}_{2}$ : A Spectroscopic Investigation. Nano Res. 2016, 9, 3622-3631. 
[19] McDonnell, S.; Addou, R.; Buie, C.; Wallace, R. M.; Hinkle, C. L. Defect-Dominated Doping and Contact Resistance in $\mathrm{MoS}_{2}$. ACS Nano 2014, 8, 2880-2888.

[20] Liu, X.; Balla, I.; Bergeron, H.; Hersam, M. C. Point Defects and Grain Boundaries in Rotationally Commensurate $\mathrm{MoS}_{2}$ on Epitaxial Graphene. J. Phys. Chem. C 2016, 120, 20798-20805.

[21] Bampoulis, P.; Bremen, R. V.; Yao, Q.; Poelsema, B.; Zandvliet, H. J. W.; Sotthewes, K. Defect Dominated Charge Transport and Fermi Level Pinning in $\mathrm{MoS}_{2} /$ Metal Contacts. ACS Appl. Mater. Interfaces 2017, 9, 19278-19286.

[22] Ghorbani-Asl, M.; Enyashin, A. N.; Kuc, A.; Seifert, G.; Heine, T. Defect-Induced Conductivity Anisotropy in MoS2 Monolayers. Phys. Rev. B 2013, 88, 245440.

[23] Lin, Z.; Carvalho, B. R.; Kahn, E.; Lv, R.; Rao, R.; Terrones, H.; Pimenta, M. A.; Terrones, M. 2D Mater. 2016, 3, 022002.

[24] Mirabelli, G.; McGeough, C.; Schmidt, M.; McCarthy, E. K.; Monaghan, S.; Povey, I. M.; McCarthy, M.; Gity, F.; Nagle, R.; Hughes, G.; Cafolla, A.; Hurley, P. K.; Duffy, R. Air Sensitivity of $\mathrm{MoS}_{2}, \mathrm{MoSe}_{2}, \mathrm{MoTe}_{2}, \mathrm{HfS}_{2}$, and HfSe2. J. Appl. Phys. 2016, 120, 125102.

[25] Zou, K.; Hong, X.; Keefer, D.; Zhu, J. Deposition of High-Quality $\mathrm{HfO}_{2}$ on Graphene and the Effect of Remote Oxide Phonon Scattering. Phys. Rev. Lett. 2010, 105, 126601.

[26] Addou, R.; Colombo, L.; Wallace, R. M. Surface Defects on Natural MoS2. ACS Appl. Mater. Interfaces 2015, 7, 11921-11929.

[27] Addou, R.; Wallace, R. M. Surface Analysis of WSe 2 Crystals: Spatial and Electronic Variability. ACS Appl. Mater. Interfaces 2016, 8, 26400-26406.

[28] Lucovsky, G.; White, R. M. Infrared-Reflectance Spectra of Layered Group-IV and Group-VI Transition-Metal Dichalcogenides. Phys. Rev. B 1973, 7, 3859-3870.

[29] Schimmel, Th.; Fuchs, H.; Sander, R.; Lux-Steiner, M. Atomically Resolved STM Imaging of Ion-Bombarded WSe 2 . Ultramicroscopy 1992, 44, 683-688. 
[30] Matthes, Th. W.; Sommerhalter, Ch.; Rettenberger, A.; Bruker, P.; Boneberg, J.; LuxSteiner, M. Ch.; Leiderer, P. Appl. Phys. A 1998, 66, S1007-S1011.

[31] Murata, H.; Kataoka, K.; Koma, A. Scanning Tunneling Microscope Images of Locally Modulated Structures in Layered Materials, $\mathrm{MoS}_{2}(0001)$ and $\mathrm{MoSe}_{2}(0001)$, Induced by Impurity Atoms. Surf. Sci. 2001, 478, 131-144.

[32] Inoue, A.; Komori, T.; Shudo, K. Atomic-Scale Structures and Electronic States of Defects on $\mathrm{Ar}^{+}$-Ion Irradiated $\mathrm{MoS}_{2}$. J. Electron Spectrosc. Relat. Phenom. 2013, 189, $11-$ 18.

[33] Kobayashi, K. Scattering Theory of Subsurface Impurities Observed in Scanning Tunneling Microscopy. Phys. Rev. B 1996, 54, 17029.

[34] Whangbo, M. H.; Ren, J.; Magonov, S. N.; Bengel, H.;Parkinson, B. A.; Suna, A. On the Correlation Between the Scanning Tunneling Microscopy Image Imperfections and Point Defects ofLayered Chalcogenides 2H-MX (M=Mo, W; X= S, Se). Surf. Sci. 1995, 326, 311-326.

[35] Ugeda, M. M.; Bradley, A. J.; Zhang, Y.; Onishi, S.; Chen, Y.; Ruan, W.; OjedaAristizabal, C.; Ryu, H.; Edmonds, M. T.; Tsai, H., et al. Characterization of Collective Ground States in Single-Layer NbSe2. Nat. Phys. 2016, 12, 92-97.

[36] Eknapakul, T.; Fongkaew, I.; Siriroj, S.; Jindata, W.; Chaiyachad, S.; Mo, S.-K.; Thakur, S.; Petaccia, L.; Takagi, H.; Limpijumnong, S.; Meevasana, W. Direct Observation of Strain-Induced Orbital Valance Band Splitting in HfSe 2 by Sodium Intercalation. Phys. Rev. B 2018, 97, 201104(R).

[37] Park, J. H.; Sanne, A.; Guo, Y.; Amani, M.; Zhang, K.; Movva, H. C. P.; Robinson, J. A.; Javey, A.; Robertson, J.; Banerjee, S. K.; Kummel, A. C. Defect Passivation of Transition Metal Dichalcogenides via a Charge Transfer van Der Waals Interface. Sci. Adv. 2017, 3, e1701661. 
[38] Rak, Z.; Mahanti, S. D.; Mandal, K. C.; Fernelius, N. C. Electronic Structure of Substitutional Defects and Vacancies in GaSe. J. Phys. Chem. Solids 2009, 70, 344-355.

[39] Hertz, H. Über die berührung fester elastischer körper. J. Reine Angew. Math. 1882, 92, $156-171$.

[40] Das, S.; Prakash, A.; Salazar, R.; Appenzeller, J. Toward Low-Power Electronics: Tunneling Phenomena in Transition Metal Dichalcogenides. ACS Nano 2014, 8, 1681-1689.

[41] Kim, C.; Moon, I.; Lee, D.; Choi, M. S.; Ahmed, F.; Nam, S.; Cho, Y.; Shin, H.; Park, S.; Yoo, W. J. Fermi Level Pinning at Electrical Metal Contacts of Monolayer Molybdenum Dichalcogenides. ACS Nano 2017, 11, 1588-1596.

[42] Peacock, P. W.; Robertson, J. Band Offsets and Schottky Barrier Heights of High Dielectric Constant Oxides. J. Appl. Phys. 2002, 92, 4712-4721.

[43] Bampoulis, P.; Teernstra, V. J.; Lohse, D.; Zandvliet, H. J. W.; Poelsema, B. Hydrophobic Ice Confined between Graphene and $\mathrm{MoS}_{2}$. J. Phys. Chem. C 2016, 120, $27079-27084$. 


\section{Conclusions and Outlook}

\subsection{General conclusions}

In this thesis, we have studied the structural and electronic properties of various 2D materials using scanning tunneling microscopy and scanning tunneling spectroscopy. Their intrinsic electronic properties are modified in various ways, such as stacking, hydrogenation, defects and coupling to substrate. The main conclusions of our work are:

In twisted bilayer graphene with a twist angle of $2.3^{\circ}$, we have found two van Hove singularities that are located near the Fermi level. We have made spatial maps of the electronic structure. The spatial maps of the electronic structure reveal a honeycomb structure, rather than a triangular structure. The origin of this honeycomb structure is due to the fact that twisted bilayer is positioned on a Bernal stacked highly-oriented pyrolytic graphite substrate.

The deposition of Si on transition metal dichalcogenides, such as $\mathrm{MoS}_{2}$ and $\mathrm{WSe}_{2}$, does not result in the growth of silicene on the transition metal dichalcogenides surfaces, but rather to the intercalation of silicon between the transition metal dichalcogenide layers.

For the first time, we have successfully synthesized germanene on a band gap material $\left(\mathrm{MoS}_{2}\right)$, which paves the way towards the determination of the intrinsic properties of this novel 2D buckled Dirac material. We found compelling evidence for the existence of charge puddles in germanene grown on a $\mathrm{MoS}_{2}$ substrate. These charge puddles are caused by charge-donating impurities in the $\mathrm{MoS}_{2}$ substrate.

Hydrogenation of germanene, synthesized on $\mathrm{Ge}_{2} \mathrm{Pt}$ nanocrystals, results in the opening of a bandgap of about $0.5 \mathrm{eV}$. This finding is an important step forward since a bandgap is a prerequisite for the realization of a germanene based field-effect transistor.

Finally, we have studied the structural and electronic properties of $\mathrm{HfSe}_{2}$. This material possesses a high density of surface defects. This high density of defects in $\mathrm{HfSe}_{2}$ degrade its electrical performance, and therefore the quality of $\mathrm{HfSe}_{2}$ crystals should be carefully checked before using this material in device applications. 


\subsection{Outlook and recommendations}

\subsubsection{Twisted graphene}

Twisted graphene layers can be found on highly-oriented pyrolytic graphite samples after cleavage. ${ }^{[1-3]}$ In our work, we also use this method to produce twisted graphene layers. Another way to prepare twisted graphene layers is by growing graphene on $4 \mathrm{H}-$ or $6 \mathrm{H}-\mathrm{SiC}$ substrates. ${ }^{[4]}$ Also in this case there is no control over the exact twist angle of the graphene. In order to produce twisted bilayer graphene with a well-defined twist angle we have built a dry transfer set up. The twisted graphene bilayers are synthesized on a h-BN/SiO 2 substrate. ${ }^{[5]}$ The setup we have built is shown in Figure 7.1a, and is referred to as 2D-transfer system. In Figure 7.1b two examples of a twisted bilayer graphene (with electrical contacts) are shown.
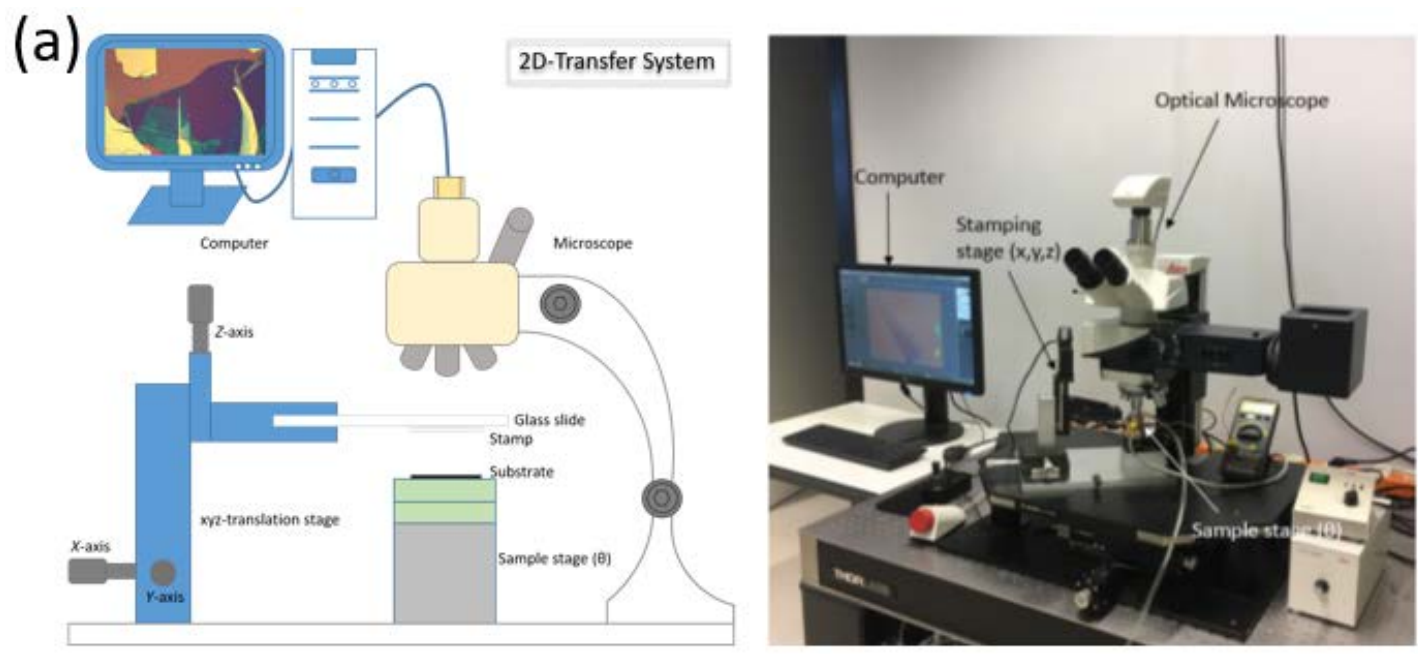

(b)
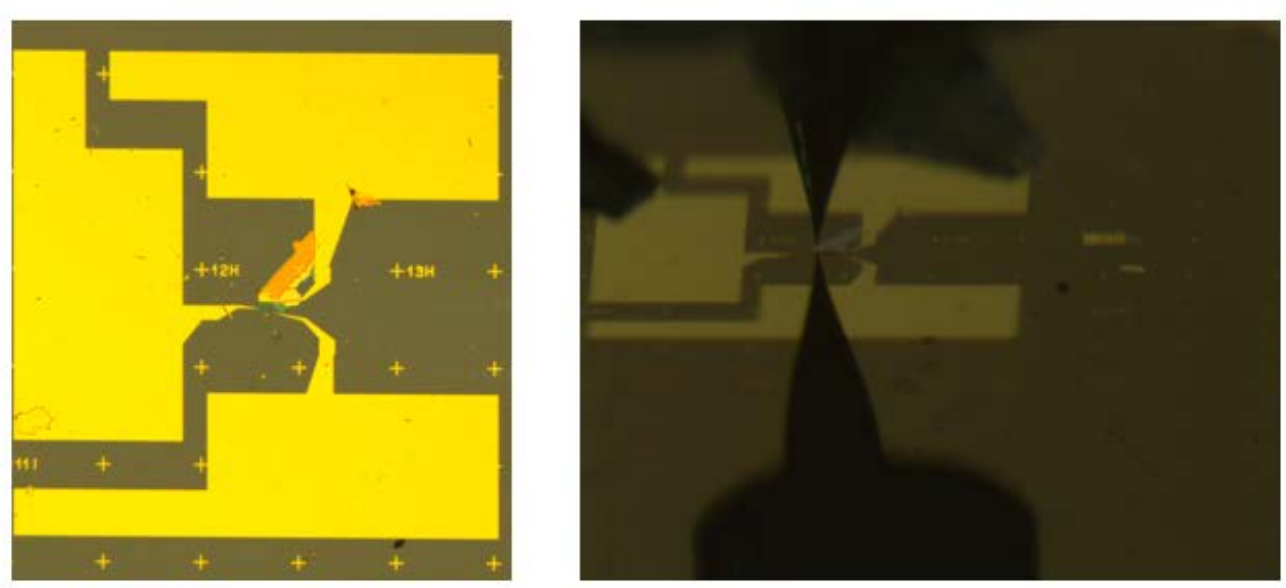

Figure 7.1: (a) Setup of the 2D-transfer system. (b) Images of twisted bilayer graphene with contacts. In the right panel the STM tip is visible. 


\subsubsection{Germanene}

Germanene has been synthesized in two different ways. In the first route we deposited Pt on a $\mathrm{Ge}(110)$ substrate and make use of the eutectic phase, which occurs a ratio of $22 \% \mathrm{Pt}$ and $78 \%$ Ge. Upon cooling down this eutectic phase separates in the two phases that are adjacent to the eutectic point in bulk phase diagram, i.e. the $\mathrm{Ge}_{2} \mathrm{Pt}$ and Ge phases, respectively. Eventually we end up with micrometer sized $\mathrm{Ge}_{2} \mathrm{Pt}$ clusters that are coated with a germanene layer. Although this system is interesting form a scientific viewpoint, the germanene coated $\mathrm{Ge}_{2} \mathrm{Pt}$ crystals are far too small for any device application.

In the second route we deposit Ge on $\mathrm{MoS}_{2}$, a transition metal dichalcogenide with a bulk bandgap of about $1.3 \mathrm{eV}$. Owing to the band gap of $\mathrm{MoS}_{2}$, the electronic states of germanene in the vicinity of the Fermi level are decoupled from the underlying substrate. The presence of defects in the $\mathrm{MoS}_{2}$ is essential since they are required for the nucleation of Ge islands. Unfortunately, these defects are often charged and thus lead to the formation of the charge inhomogeneities, i.e. charge puddles, in the germanene layer. These charge puddles have a negative effect on the electronic properties of germanene since they lead to reduction of the charge carrier mobilities.

We suggest to grow germanene on hexagonal boron nitride. Hexagonal boron nitride has a large bandgap of about $6 \mathrm{eV}$, is nearly defect-free and has a honeycomb lattice. In addition, the nearest-neighbor distance between the Ge atoms in the germanene honeycomb structure (2.5 $\AA$ ) perfectly matches with the lattice constant of hexagonal boron nitride. 


\section{Bibliography}

[1] Li, G.; Luican, A.; Lopes dos Santos, J. M. B.; Castro Neto, A. H.; Reina, A.; Kong, J.; Andrei, E. Y. Observation of Van Hove Singularities in Twisted Graphene Layers. Nat. Phys. 2010, 6 (2), 109-113.

[2] Yin, L.; Qiao, J.; Wang, W., et al. Tuning Structures and Electronic Spectra of Graphene Layers with Tilt Grain Boundaries. Phys. Rev. B 2014, 89, 205410.

[3] Yin, L.; Qiao, J.; Zuo, W.; Li, W.; He, L. Experimental Evidence for Non-Abelian Gauge Potentials in Twisted Graphene Bilayers. Phys. Rev. B 2015, 92, 081406(R).

[4] Brihuega, I.; Mallet, P.; González-Herrero, H.; Trambly de Laissardière, G.; Ugeda, M. M.; Magaud, L.; Gómez-Rodríguez, J. M.; Ynduráin, F.; Veuillen, J.-Y. Unraveling the Intrinsic and Robust Nature of van Hove Singularities in Twisted Bilayer Graphene by Scanning Tunneling Microscopy and Theoretical Analysis. Phys. Rev. Lett. 2012, 109 (19), 196802.

[5] Cao, Y.; Fatemi, V.; Fang, S.; Watanabe, K.; Taniguchi, T.; Kaxiras, E.; Jarillo-Herrero, P. Unconventional Superconductivity in Magic-Angle Graphene Superlattices. Nature 2018, $556,43-50$. 


\section{Summary}

This thesis deals with the two-dimensional materials. Soon after the discovery of graphene, the first two-dimensional material, many other two-dimensional materials have been synthesized and characterized.

In chapter 3 we studied the structural and electronic properties of a twisted graphene. The top layer of Bernal stacked highly-oriented pyrolytic graphite sample was twisted by an angle of $2.3^{\circ}$. This twist angle results in a moiré pattern with a periodicity of $6.2 \mathrm{~nm}$. The twisted graphene layer has electronic properties that are distinctly different from that of a single layer graphene due to the nonzero interlayer coupling. The integrated differential conductivity spectrum exhibits two well-defined van Hove singularities, one located just above the Fermi level and the other just below the Fermi level. Spatial maps of the differential conductivity that are recorded at energies near the Fermi level exhibit a honeycomb structure that is comprised of two inequivalent hexagonal sublattices. For energies near the Fermi level the hexagonal structure in the differential conductivity maps vanishes. We have performed tight-binding calculations of the twisted graphene system using the propagation method, in which a third graphene layer is added to mimic the substrate. This third layer lowers the symmetry and explains the development of the two hexagonal sublattices in the moiré pattern. Our experimental results are in excellent agreement with the tight-binding calculations.

In chapter 4 and 5 we have studied the two novel elemental two-dimensional materials that are based on silicon and germanium, respectively. Owing to their $s p^{2}$ type of electronic structure the elements of the 'carbon' column of the periodic system i.e. silicon, germanium and tin are of particular of interest as potential two-dimensional materials. The silicon and germanium analogues of graphene are referred to as silicene and germanene, respectively, and share many properties with graphene. In thesis we have attempted to synthesize these materials. Silicon was deposited on $\mathrm{WSe}_{2}$, a transition metal dichalcogenide with an indirect bandgap of about 1.2-1.3 $\mathrm{eV}$. After the deposition of silicon the $\mathrm{WSe}_{2}$ transform from an atomically flat substrate to a substrate that exhibit a hill-and-valley structure. Since the lattice constant of the corrugated $\mathrm{WeS}_{2}$ substrate is exactly same as that of the flat substrate we conjectured that the deposited $\mathrm{Si}$ atoms have been intercalated between the WSe 2 layers. In order to validate this conjecture we have recorded spatial maps of $d I / d z$. The $d I / d z$ signal is proportional to the square root of the work function and does not contain any topographic information. The homogeneous $d I / d z$ map provides compelling evidence that the top layer is indeed pure $\mathrm{WSe}_{2}$. 
In chapter 5 we focus on the other elemental two-dimensional material, germanene. Germanene has been successfully synthesized on $\mathrm{Ge}_{2} \mathrm{Pt}$ and $\mathrm{MoS}_{2}$ substrates. The density of states of the germanene layer exhibits a well-defined V shape around the Fermi level, which hints to a twodimensional Dirac system. We found that germanene grown on $\mathrm{MoS}_{2}$ substrate exhibits charge puddles, analogous to the charge puddles which have been reported for graphene on $\mathrm{SiO}_{2}$. $\mathrm{By}$ using the Dirac point mapping technique were able to map out the charge puddles in real space. The Dirac point varies from $-30 \mathrm{meV}$ to $+15 \mathrm{meV}$ for the germanene/ $\mathrm{MoS}_{2}$ system, corresponding to a charge density in the puddles in the range of $2.6 \times 10^{-3}$ electrons to $6.6 \times 10^{-4}$ holes per $\mathrm{nm}^{2}$. The radius of these puddles is about $10-20 \mathrm{~nm}$, resulting in a total charge of the order of one charge carrier per puddle. The defect concentration in the top layer of the $\mathrm{MoS}_{2}$ substrate is very comparable to the density of charge puddles, suggesting that the charge puddles are caused by the charged defects in the top layer of the $\mathrm{MoS}_{2}$ substrate. In addition, we have studied the hydrogenation of germanene synthesized on $\mathrm{Ge}_{2} \mathrm{Pt}$ crystals using scanning tunneling microscopy and spectroscopy. The germanene honeycomb lattice is buckled and consists of two hexagonal sub-lattices that are slightly displaced with respect to each other. The hydrogen atoms adsorb exclusively on the Ge atoms of the upward buckled hexagonal sublattice. At a hydrogen exposure of about $100 \mathrm{~L}$, the $(1 \times 1)$ buckled honeycomb structure of germanene converts to a $(2 \times 2)$ structure. Scanning tunneling spectra recorded on this $(2 \times 2)$ structure reveal the opening of a bandgap of about $0.2 \mathrm{eV}$. A fully (half) hydrogenated germanene surface is obtained after an exposure of about $9000 \mathrm{~L}$ hydrogen. The hydrogenated germanene, also referred to as germanane, has a sizeable bandgap of about $0.5 \mathrm{eV}$ and is slightly n-type.

In chapter 6, the defects and air-stability of hafnium diselenide ( $\mathrm{HfSe}_{2}$ ) have been studied. $\mathrm{HfSe}_{2}$ is a very appealing material for field-effect based applications owing to its moderate band gap of about $1 \mathrm{eV}$ and its high- $\kappa$ dielectric native oxide. Unfortunately, the experimentally determined charge carrier mobility is about 3 orders of magnitude lower than the theoretically predicted value. This strong deviation calls for a detailed investigation of the physical and electronic properties of $\mathrm{HfSe}_{2}$. Here, we have studied the structure, density, and density of states of several types of defects that are abundant on the $\mathrm{HfSe}_{2}$ surface using scanning tunneling microscopy and spectroscopy. Compared to $\mathrm{MoS}_{2}$ and $\mathrm{WSe}_{2}, \mathrm{HfSe}_{2}$ exhibits similar type of defects, albeit with a substantially higher density of $9 \times 10^{11} \mathrm{~cm}^{-2}$. The most abundant defect is a subsurface defect, which shows up as a dim feature in scanning tunneling microscopy images. These dim dark defects have a substantially larger band gap $(1.25 \mathrm{eV})$ than the pristine surface 
$(1 \mathrm{eV})$, suggesting a substitution of the Hf atom by another atom. The high density of defects on the $\mathrm{HfSe}_{2}$ surface leads to very low Schottky barrier heights. Conductive atomic force microscopy measurements reveal a very small dependence of the Schottky barrier height on the work function of the metals, suggesting a strong Fermi-level pinning. We attribute the observed Fermi-level pinning (pinning factor $\sim 0.1$ ) to surface distortions and Se/Hf defects. In addition, we have also studied the $\mathrm{HfSe}_{2}$ surface after the exposure to air by scanning tunneling microscopy and conductive atomic force microscopy. Partly oxidized layers with bandgaps of $2 \mathrm{eV}$ and Schottky barrier heights of $\sim 0.6 \mathrm{eV}$ were readily found on the surface. Our experiments reveal that $\mathrm{HfSe}_{2}$ is very air-sensitive, implying that capping or encapsulating of $\mathrm{HfSe}_{2}$, in order to protect it against oxidation, is a necessity for technological applications. 


\section{Samenvatting}

In dit proefschrift heeft betrekking op tweedimensionale materialen. Kort na de ontdekking van grafeen, het eerste tweedimensionale materiaal, zijn vele andere tweedimensionale materialen gesynthetiseerd en gekarakteriseerd. In dit proefschrift zullen we een aantal van deze nieuwe tweedimensionale materialen onder de loep nemen.

In hoofdstuk 3 hebben we de structurele en elektronische eigenschappen van gedraaid grafeen bestudeerd. De bovenste laag van Bernal gestapeld hoog-georiënteerd pyrolytisch grafietmonster werd gedraaid met een hoek van $2,3^{\circ}$. Deze draaihoek resulteert in een moirépatroon met een periodiciteit van 6,2 nm. De gedraaide grafeenlaag heeft elektronische eigenschappen die duidelijk verschillen van die van een enkele laag grafeen vanwege de koppeling tussen de lagen. Het geïntegreerde differentiële geleidbaarheidsspectrum (dI/dV) vertoont twee goed gedefinieerde van Hove-singulariteiten, één die zich net boven het Ferminiveau bevindt en de andere vlak onder het Fermi-niveau. Ruimtelijke maps van de differentiële geleidbaarheid die worden geregistreerd bij energieën nabij het Fermi-niveau vertonen een honingraatstructuur die bestaat uit twee ongelijke hexagonale subroosters. Voor energieën in de buurt van het Fermi-niveau verdwijnt de hexagonale structuur in de maps van differentiële geleidbaarheid. We hebben tight-binding berekeningen uitgevoerd aan het gedraaide grafeensysteem met behulp van de propagatiemethode, waarbij een derde grafeenlaag wordt toegevoegd om het substraat na te bootsen. Deze derde laag verlaagt de symmetrie en verklaart de ontwikkeling van de twee hexagonale subroosters in het moiré-patroon. Onze experimentele resultaten zijn in uitstekende overeenstemming met de tight-binding berekeningen.

In hoofdstuk 4 en 5 hebben we de twee nieuwe elementaire tweedimensionale materialen bestudeerd die respectievelijk zijn gebaseerd op silicium en germanium. Vanwege hun $\mathrm{sp}^{2}$ type elektronische structuur zijn de elementen van de 'koolstof' kolom van het periodieke systeem, d.w.z. silicium en germanium, interessant als potentiële tweedimensionale materialen. De grafeen-achtige allotropen van silicium en germanium worden respectievelijk aangeduid als siliceen en germaneen en delen veel eigenschappen met grafeen. In het proefschrift hebben we geprobeerd deze materialen te synthetiseren. Silicium werd gedeponeerd op WSe2, een overgangsmetaal dichalcogenide met een indirecte bandgap van ongeveer 1.2-1.3 eV. Na de depositie van silicium transformeert het WSe 2 van een atomair vlak substraat naar een substraat dat een heuvel-en-dal structuur vertoont. Omdat de roosterconstante van het gegolfde $\mathrm{WeS}_{2}$ substraat exact hetzelfde is als die van het vlakke substraat, veronderstelden we dat de 
gedeponeerde Si-atomen tussen de $\mathrm{WSe}_{2}$ lagen zijn geïntercaleerd. Om dit vermoeden te valideren, hebben we ruimtelijke maps van $\mathrm{dI} / \mathrm{dz}$ opgenomen. Het $\mathrm{dI} / \mathrm{z}$-signaal is evenredig met de wortel van de werkfunctie en bevat geen topografische informatie. De homogene dI/dzmap biedt overtuigend bewijs dat de bovenste laag inderdaad puur WSe 2 is.

In hoofdstuk 5 concentreren we ons op het andere elementaire tweedimensionale materiaal, germaneen. Germaneen is met succes gesynthetiseerd op $\mathrm{Ge}_{2} \mathrm{Pt}$ en $\mathrm{MoS}_{2}$ substraten. De dichtheid van toestanden van de germaneenlaag vertoont een goed gedefinieerde V-vorm rond het Fermi-niveau, die hint naar een tweedimensionaal Dirac-systeem. We ontdekten dat germeen gegroeid op $\mathrm{MoS}_{2}$-substraat charge puddles vertoont, analoog aan decharge puddles die zijn gerapporteerd voor grafeen op $\mathrm{SiO}_{2}$. Door het gebruik van de Dirac-punt mappingstechniek konden de charge puddles ruimtelijk in kaart worden gebracht. Het Diracpunt varieert van -30 meV tot $+15 \mathrm{meV}$ voor het germaneen / $\mathrm{MoS}_{2}$-systeem, wat overeenkomt met een ladingsdichtheid in de charge puddles in het bereik van $2.6 \times 10^{-3}$ elektronen tot $6.6 \mathrm{x}$ $10^{-4}$ gaten per $\mathrm{m}^{2}$. De straal van deze charge puddles is ongeveer $10-20 \mathrm{~nm}$, wat resulteert in een totale lading in de orde van één ladingsdrager per puddle. De defectconcentratie in de toplaag van het $\mathrm{MoS}_{2}$ substraat is zeer vergelijkbaar met de dichtheid van charge puddles, hetgeen suggereert dat de charge puddles worden veroorzaakt door de geladen defecten in de toplaag van het $\mathrm{MoS}_{2}$ substraat. Daarnaast hebben we de hydrogenering van germaneen gesynthetiseerd op $\mathrm{Ge}_{2} \mathrm{Pt}$ kristallen bestudeerd met behulp van scanning tunneling microscopie en spectroscopie. Het germaneen honingraatrooster is buckled en bestaat uit twee zeshoekige subroosters die enigszins ten opzichte van elkaar zijn verplaatst. De waterstofatomen adsorberen exclusief op de Ge-atomen van het opwaarts gebuckled zeshoekige subrooster. Bij een waterstofblootstelling van ongeveer $100 \mathrm{~L}$ zet de $(1 \mathrm{x}$ 1) gebuckled honingraatstructuur van germaneen zich om in een $(2 \times 2)$ structuur. Scanning tunneling spectra die zijn opgenomen op deze $(2 \times 2)$ structuur onthullen de opening van een bandgap van ongeveer 0,2 eV. Een volledig (half) gehydrogeneerd germaneenoppervlak wordt verkregen na een blootstelling van ongeveer $9000 \mathrm{~L}$ aan waterstof. Het gehydrogeneerde germaneen, ook aangeduid als germanaan, heeft een aanzienlijke bandgap van ongeveer $0.5 \mathrm{eV}$ en is enigszins n-type.

In hoofdstuk 6 zijn de defecten en luchtstabiliteit van hafniumdiselenide ( $\left.\mathrm{HfSe}_{2}\right)$ bestudeerd. $\mathrm{HfSe}_{2}$ is een zeer aantrekkelijk materiaal voor toepassingen op basis van veldeffecten dankzij de moderate bandgap van ongeveer $1 \mathrm{eV}$ en het hoge K-diëlektrische natuurlijke oxide. Helaas is de experimenteel bepaalde ladingdragersmobiliteit ongeveer 3 ordes van grootte lager dan de theoretisch voorspelde waarde. Deze sterke afwijking vereist een gedetailleerd onderzoek van 
de fysieke en elektronische eigenschappen van $\mathrm{HfSe}_{2}$. Hier hebben we de structuur, dichtheid en dichtheid van toestanden van verschillende soorten defecten bestudeerd die overvloedig aanwezig zijn op het HfSe 2 oppervlak met behulp van scanning tunneling microscopie en spectroscopie. Vergeleken met $\mathrm{MoS}_{2}$ en $\mathrm{WSe}_{2}$ vertoont $\mathrm{HfSe}_{2}$ soortgelijke type defecten, zij het met een aanzienlijk hogere dichtheid van $9 \times 10^{11} \mathrm{~cm}^{-2}$. Het meest voorkomende defect is een subsurface defect, dat zich voordoet als een dim protusion in e tunneling microscopiebeelden. Deze zwakke donkere defecten hebben een aanzienlijk grotere bandafstand $(1.25 \mathrm{eV})$ dan het oorspronkelijke oppervlak $(1 \mathrm{eV})$, wat duidt op een substitutie van het Hf-atoom door een ander atoom. De hoge dichtheid van defecten op het $\mathrm{HfSe}_{2}$ oppervlak leidt tot zeer lage Schottky-barrièrehoogten. Geleidende atomaire krachtmicroscopie metingen onthullen een zeer kleine afhankelijkheid van de Schottky-barrièrehoogte op de werkfunctie van de metalen, wat duidt op een sterke fixatie op Fermi-niveau. We schrijven de waargenomen Fermi-level pinning (pinning factor $~ 0.1$ ) toe aan oppervlaktevervormingen en Se/Hf defecten. Daarnaast hebben we ook het HfSe 2 oppervlak bestudeerd na blootstelling aan lucht gebuikmakend van scanning tunneling microscopie en geleidende atomaire krachtmicroscopie. Gedeeltelijk geoxideerde lagen met bandafstanden van 2 eV en Schottky barrièrehoogtes van $\sim 0.6 \mathrm{eV}$ werden gevonden op het oppervlak. Onze experimenten laten zien dat $\mathrm{HfSe}_{2}$ zeer luchtgevoelig is, wat betekent dat het bedekken of coaten van $\mathrm{HfSe}_{2}$, om het te beschermen tegen oxidatie, een noodzaak is voor technologische toepassingen. 


\section{List of publications}

- Q. Yao, Z. Jiao, P. Bampoulis, L. Zhang, A. N. Rudenko, M. I. Katsnelson, H. J. W. Zandvliet. Charge Puddles in Germanene. Applied Physics Letters 114, 041601 (2019).

- Q. Yao, L. Zhang, P. Bampoulis, and H. J. W. Zandvliet. Nanoscale Investigation of Defects and Oxidation of HfSe 2 . The Journal of Physical Chemistry C 122, 25498 (2018).

- Q. Yao, L. Zhang, N. S. Kabanov, A. N. Rudenko, T. Arjmand, H. Rahimpour Soleimani, A. L. Klavsyuk, and H. J. W. Zandvliet. Bandgap opening in hydrogenated germanene. Applied Physics Letters 112, 171607 (2018).

- Q. Yao, R. van Bremen, G. J. Slotman, L. Zhang, S. Haartsen, K. Sotthewes, P. Bampoulis, P. L. de Boeij, A. van Houselt, S. Yuan, and H. J. W. Zandvliet. Spatially resolved electronic structure of twisted graphene. Physical Review B 95, 245116 (2017).

- Q. Yao, R. van Bremen, and H. J. W. Zandvliet. Growth of silicon on tungsten diselenide. Applied Physics Letters 109, 243105 (2016).

- $\quad$ R. van Bremen*, Q. Yao*, S. Banerjee, D. Cakir, N. Oncel, and H. J. W. Zandvliet. Intercalation of Si Between MoS2 Layers. Beilstein Journal of Nanotechnology 8, 1952 (2017).

- L. Zhang, P. Bampoulis, A. N. Rudenko, Q. Yao, A. van Houselt, B. Poelsema, M. I. Katsnelson, and H. J. W. Zandvliet. Structural and Electronic Properties of Germanene on $M_{o}$. Physical Review Letters 116, 256804 (2016).

- P. Bampoulis, R. van Bremen, Q. Yao, B. Poelsema, H. J. W. Zandvliet, K. Sotthewes. Defect Dominated Charge Transport and Fermi Level Pinning in $\mathrm{MoS}_{2} / \mathrm{Metal}$ Contacts. ACS Applied Materials \& Interfaces 9, 19278 (2017).

- Z. Jiao, Q. Yao, L. M. Balescu, Q. Liu, B. Tang, H. J. W. Zandvliet. Structural and electronic properties of the $\alpha$-GeSe surface. Surface Science 686, 17 (2019).

- P. Bampoulis, L. Zhang, Q. Yao, R. van Breman, C. J. Walhout, A. Acun, A. van Houselt, and H. J. W. Zandvliet. Germanene: Silicene's twin sister. (Book Chapter, Silicene: prediction, synthesis, application, 255-267). 


\section{Acknowledgements}

This thesis would not be possible without the help and support of many individuals, to whom I owe my gratitude. In this part, I would like to thank and acknowledge them for their support, collaboration and comfort.

First of all, I would like to express my sincere thanks from the bottom of my heart to my supervisor Prof. Harold Zandvliet. Harold, I truly appreciate the opportunity you gave to me to join PIN group as a PhD student and continuous support throughout my PhD study. Thank you so much for giving me the freedom and trust to carry out my ideas and for giving me courage to go on. Your immense knowledge, patience, and considerate behavior impressed me a lot. Your guidance helped me in all the time of research and writing of this thesis. I could not have imagined having a better supervisor for my $\mathrm{PhD}$ study.

I would also like to thank a lot to the other staff in PIN group. Dr. Arie, thank you so much for your kind help in the last four years. It is my pleasure that you are also the committee member of my defense. I like your jokes always although I did not totally understand them sometimes. Dr. Kai, your encouragement and helps impressed me a lot, many thanks to such a gentle man. Dr. Stefan, I really enjoyed the group outing you arranged together with other committee members (Kai and Simone) each time. I got a lot of fun from all the nice trips, thank you very much. Martin and Hans, the technical staff of the group, thanks to both of you for your innumerable help to order the experimental materials and fix the experimental devices. I appreciate your trouble-shooting in the lab, since I am often a trouble-maker. I would like to thank Simone, the secretary of the group, for her care and help in the past four years.

Prof. B. Poelsema, Prof. M.A. Stöhr, Prof. O. Gurlu, Prof. J.W.M. Hilgenkamp and Prof. J.E. ten Elshof: I would like to thank you for agreeing to be members of my graduation committee and thoroughly reviewing my thesis.

I would like to acknowledge the China Scholar Council for the financial support of this research.

I thank my following group colleagues for the scientific and non-scientific discussions, and for all the fun we have had in the last four years. I acknowledge Rik and Xingchen to be my paranymphs.

Rik, thanks for the fruitful and fun collaboration. I really enjoy working and having sports with you. We had a lot of fun from tennis, badminton, beach volleyball and ping-pong. I am always 
happy when I do the experiments together with you and Dr. Kai, because I know we are going to make our experimental plan be true. Thank you so much for your help with LT-STM measurement. I wish you all the best.

Xingchen, as a master student, you are smart and hard-working. I appreciate having you as my student. Since you have a chance to start your PhD career, I would like to express my best wishes to you.

Lijie, many thanks for the care and fruitful collaboration during my first two years in PIN group. I still remember that we had a wonderful trip in Italy after the graphene conference in Genoa. I wish your family to grow and always be happy. Zhen and Zhiguo, my fellow countrymen, thank you very much for your help in Enschede, and I hope you will publish a lot of nice papers in PIN group and wish both of you to enjoy your life of study abroad.

My utmost appreciation goes to Edwin, Pantelis, Adil, Özlem, Tabassom, Nikolai, Carolien, Wojciech, Martina, Jorn, Gerjan, Thomas, Ruben, Maarten, Daniel, Valent, and all the past and present members of PIN group, for helpful and pleasant working environment.

Thanks to my friends, Lianci in Utrecht University, Yan in Queen Mary University of London and Mengjiao in RWTH Aachen University, for all the happy time we had together. We knew each other and became friends in China. I truly appreciate our friendship. Thank you so much and best wishes to all of you.

I would also like to thank the Chinese friends I meet in Enschede, Qian Li, Shu Zhang, Shuqin Cao, Chuan Li, Shuijing Jie, Jianfeng Huang, Jun Wang, Yan Liu, Yang Wang, Sizhao Huang, Zhengchao Guo and many more, for all the moments we have shared.

I want to thank my parents, Tingbo and Lianxiang, and my sister, Qijiao, for their comfort, support and unconditional love. I would also like to take this opportunity to express my gratitude to my uncle, Tingwu. Without your help, I maybe even did not have the chance to study further after my primary school.

At last, my deepest gratitude goes to my girlfriend, Zhenping. I appreciate everything you did for me. I feel lucky to have you in my life. Thank you very much for everything! 


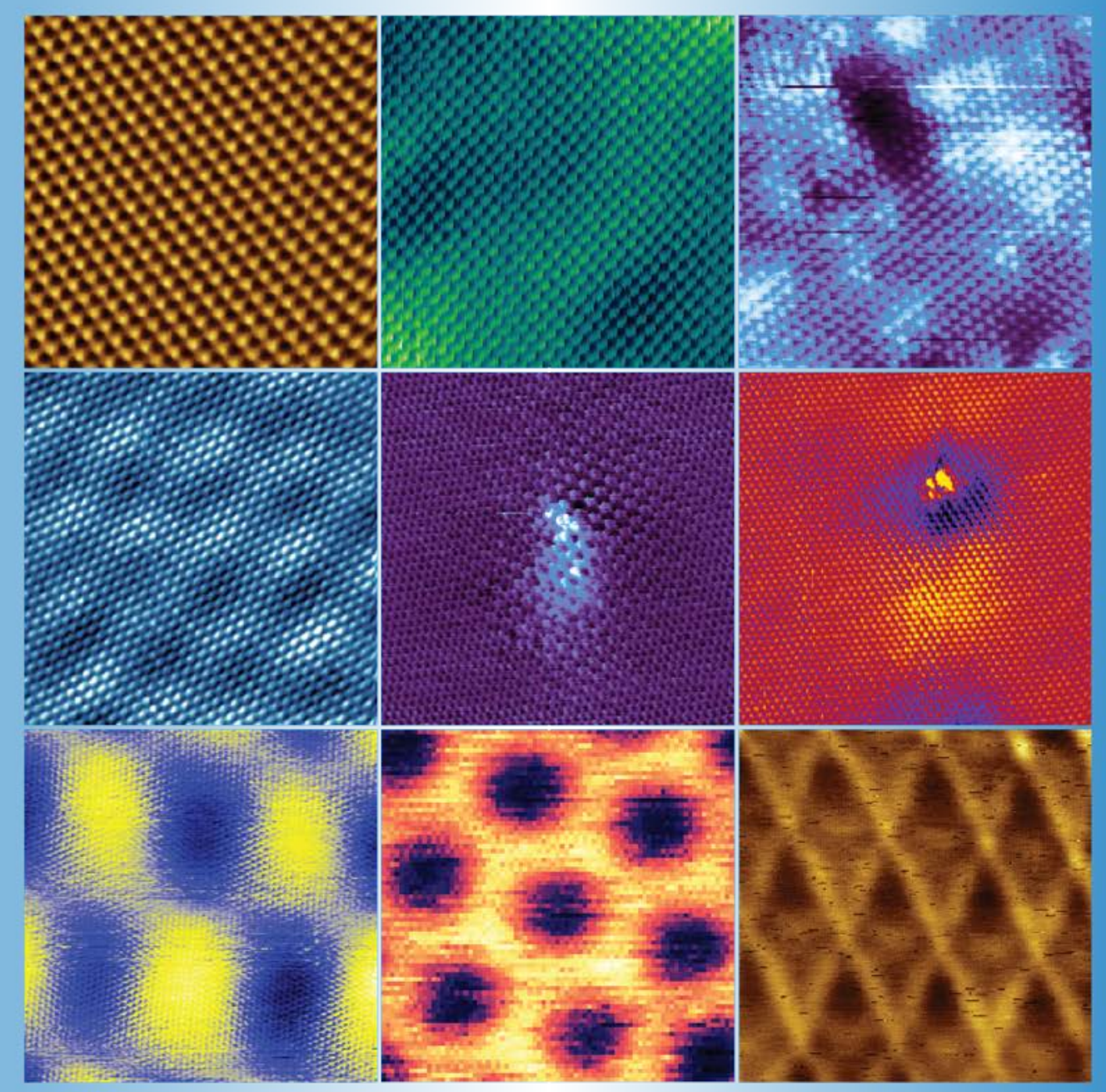

